\title{
Data Driven Trigger Design and Analysis for the NOvA experiment
}

\author{
A Thesis presented to the Graduate Faculty \\ of the University of Virginia in Candidacy for the Degree of \\ Masters of Science in Physics \\ by \\ Serdar Kurbanov \\ University of Virginia \\ November 08, 2016
}

On my honor as a University student, on this assignment I have neither given nor received unauthorized aid as defined by the Honor Guidelines for Papers in Science, Technology, and Society Courses.

\section{(Full signature)}

Approved

(Science,

Technology, and

Society Advisor)

- Craig Robert Group (Signature) 


\section{Abstract}

This thesis primarily describes analysis related to studying the Moon shadow with cosmic rays, an analysis using upward-going muons trigger data, and other work done as part of MSc thesis work conducted at Fermi National Laboratory. While at Fermilab I made hardware and software contributions to two experiments - NOvA and Mu2e.

NOvA is a neutrino experiment with the primary goal of measuring parameters related to neutrino oscillation. This is a running experiment, so it's possible to provide analysis of real beam and cosmic data. Most of this work was related to the Data-Driven Trigger (DDT) system of NOvA. The results of the Upward-Going muon analysis was presented at ICHEP in August 2016. The analysis demonstrates the proof of principle for a low-mass dark matter search.

Mu2e is an experiment currently being built at Fermilab. Its primary goal is to detect the hypothetical neutrinoless conversion from a muon into an electron. I contributed to the production and tests of Cathode Strip Chambers (CSCs) which are required for testing the Cosmic Ray Veto (CRV) system for the experiment. This contribution is described in the last chapter along with a short description of the technical work provided for the DDT system of the NOvA experiment.

All of the work described in this thesis will be extended by the next generation of UVA graduate students and postdocs as new data is collected by the experiment. I hope my efforts of have helped lay the foundation for many years of beautiful results from Mu2e and NOvA. 


\section{ACKNOWLEDGEMENTS}

I personally would like to acknowledge my scientific advisor Craig Group for advisory and directions, UVA faculty for perfect lectures and NOvA collaboration for productive team work. I would specially thank my collaborators on upward-going studies: Cristiana Principato (UVA graduate student), Rob Mina (UVA undergraduate student, now Caltech graduate student), Aristeidis Tsaris (Fermilab postdoc), Leonidas Aliaga (Fermilab postdoc) and Andrew Norman (Scientist 1 at Fermilab). 


\section{Contents}

Abstract

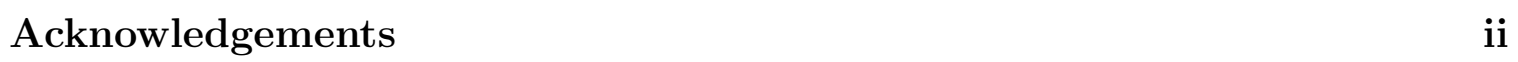

Contents

List of Tables $\quad$ V

List of Figures $\quad$ vi

List of Abbreviations $\quad \mathrm{X}$

1 Introduction 1

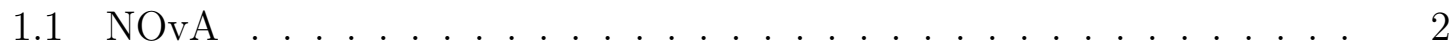

1.2 Mu2e . . . . . . . . . . . . . . . . . . . . . 7

2 Moon Shadow Studies 10

2.1 Motivation . . . . . . . . . . . . . . . . . . . . . . . . . . 10

2.2 DDT Moon and Sun trigger $\ldots \ldots$. . . . . . . . . . . . . . . 10

2.3 Acceptance and fiducial volume . . . . . . . . . . . . . . . . . . . . . 11

2.4 Smart prescale . . . . . . . . . . . . . . . . . . . . . . 13

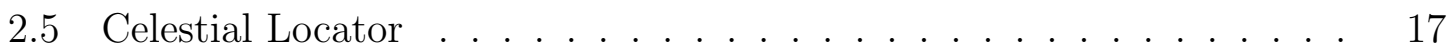

2.6 Moon shadow . . . . . . . . . . . . . . . . . . . . . . . . . . . 18

2.7 Current state and plans . . . . . . . . . . . . . . . . . . . . . . 18

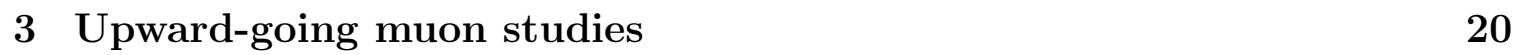

3.1 Motivation . . . . . . . . . . . . . . . . . 20

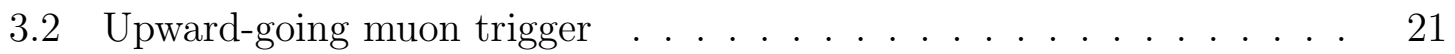

3.3 Timing resolution . . . . . . . . . . . . . . . . . . . . . . . . . . . . . 22

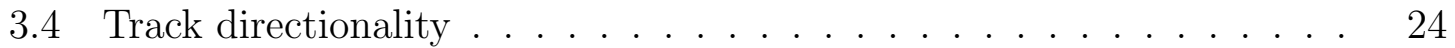

3.5 Analysis . . . . . . . . . . . . . . . . . . . . . . . . . . . . . . . 27

3.6 ICHEP 2016 . . . . . . . . . . . . . . . . . . . . . . . . . . . . . . . 28

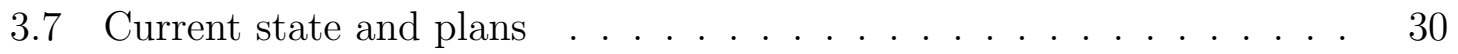

$\begin{array}{lll}4 & \text { DDT event display } & 32\end{array}$

4.1 Motivation . . . . . . . . . . . . . . . . . . . . . . . 32

4.2 Choice of technologies . . . . . . . . . . . . . . . . . 33 
4.3 DDT and offline modules . . . . . . . . . . . . . . . . . . . . . . . 34

4.4 Use cases $\ldots \ldots \ldots \ldots$

4.5 Current state and plans . . . . . . . . . . . . . . . . . 36

$\begin{array}{lll}5 & \text { Other } & 38\end{array}$

5.1 Mu2e: CSC production and testing . . . . . . . . . . . . . . 38

5.2 Management for novasoft builds . . . . . . . . . . . . . . . . . . . . 41

$\begin{array}{lll}6 & \text { Summary } & 43\end{array}$

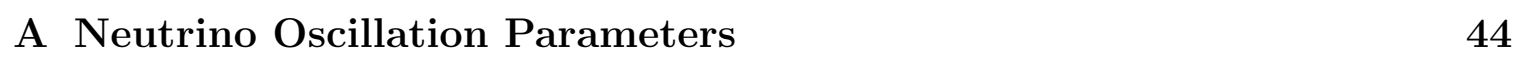

B NOvA FD Exposure and Volume

\begin{tabular}{ll|}
\hline C Moon Trigger Rates & 48
\end{tabular}

D NOVAS and Celestial Locator Code 50

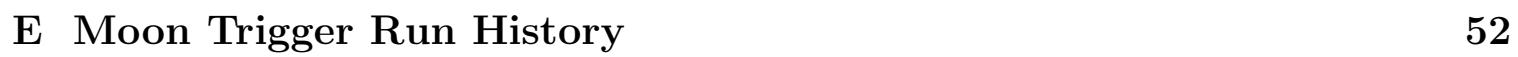

F Upward Going Muons Rate Estimate 54

$\begin{array}{ll}\text { G DDT Efficiency and Purity } & 58\end{array}$

\begin{tabular}{ll}
\hline H UpMu Data Quality & 63
\end{tabular}

\begin{tabular}{lll}
\hline I UpMu Cut Parameters & 65
\end{tabular}

\begin{tabular}{|lll}
\hline J & DDT Event Display Design and Usage & 68
\end{tabular}

\begin{tabular}{ll}
\hline K CSC Anatomy & 71
\end{tabular}

L The CSC Noise, Efficiency and Amplitudes 73 


\section{LIST OF TABLES}

5.1 Table of maximal working voltages (in $V$ ) for produced cathode strip

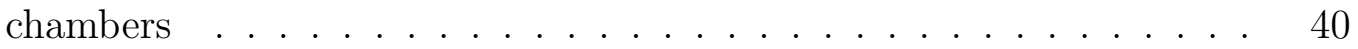

A.1 Current estimates for parameters of neutrino oscillations . . . . . . . 44

G.1 The DDT system efficiency of the track reconstruction when compared to different offline reconstruction algorithms. . . . . . . . . . . . . . . 58

I.1 Selection criteria used by the upward-going through-going muon trigger. Cuts are designed to select muon tracks with lengths and numbers of hits sufficient to compute reliable likelihood values for track direction. 65

I.2 Track quality cuts (tracks got with window tracker algorithm). Cuts are designed to select muon tracks with lengths and numbers of hits sufficient to compute reliable likelihood values for track direction. . . 66

I.3 Efficiency of track quality cuts. Data is taken from the minimum bias cosmic data stream. . . . . . . . . . . . . . . . . . . . 66

I.4 Track directionality cuts (tracks got with window tracker algorithm). Together with track quality cuts they form the full set of cuts to determine track direction. . . . . . . . . . . . . . . . . . 66

I.5 Efficiency of the cuts . . . . . . . . . . . . . . . . . . 67

\begin{tabular}{lll}
\hline I.6 & The efficiency of cuts applied to the upward-going muon data sample. & 67
\end{tabular} 


\section{List OF FigURES}

1.1 Fermi National Accelerator Laboratory . . . . . . . . . . . . . . . . . 1

1.2 Location of Fermilab and NOvA Far Detector . . . . . . . . . . . . . 2

1.3 Neutrino mass hierarchy. . . . . . . . . . . . . . . . . . . . 4

1.4 The NOvA Near and Far Detectors . . . . . . . . . . . . . . . . . . . 5

1.5 From left to right: PVC, fibers and avalanche photodiodes which form cells of NOvA detectors . . . . . . . . . . . . . 6

$1.6 \quad$ First neutrino candidate in 2 views of the NOvA FD event display . . 6

1.7 Signal and background processes for the Mu2e experiment . . . . . . 7

1.8 The expected spectrum of electron energies at the Mu2e detector. DIO stands for the decay in orbit process . . . . . . . . . . . . . . . 8

1.9 Design of the Mu2e experiment . . . . . . . . . . . . . . . . . . . . 8

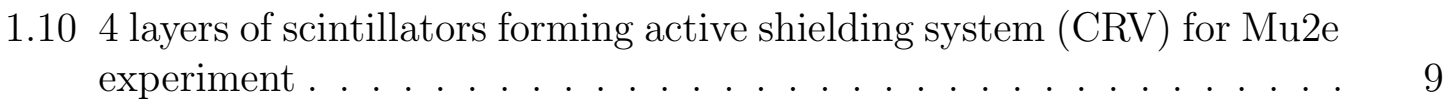

2.1 Preliminary Moon shadow analysis done by C. Backhouse. The deficit in the cosmic particles rate shows shadowing of particle flux by the Moon. ....................... 11

2.2 Rate of cosmic particle tracks triggered by Moon trigger with cut for false tracks. The Moon elevation is marked by dark circles. Data taken from cosmic pulser files of Jun 282015 . . . . . . . . . . . . . . . . . 11

2.3 Rate of cosmic particle tracks triggered by Sun trigger with cut for false tracks. The Sun elevation is marked by yellow circles. Data taken from cosmic pulser files of Jun 282015 . . . . . . . . . . . . . . . . 12

2.4 Cosmic rate distribution in NOvA Far Detector . . . . . . . . . . . . 12

2.5 The scheme for the calculation of fiducial volume of the Far Detector. Only volume capable of producing registered particle with given length cut is included as fiducial. Volume by sides of the detector is not active. 13

2.6 The cheme for calculation of effective acceptance of the Far Detector. Only surface capable of producing registered particle with given length cut is included as effective. Surface by corners of the detector is not

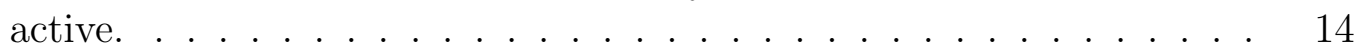

2.7 Acceptance of the Far Detector with $1 m$ cut multiplied by factor of $\cos ^{2}(\theta)$ from zenith angle flux dependence. Values in $m^{2}$. . . . . . . 14

2.8 The expected rate of cosmics collected by the Moon trigger within a $5^{\circ}$ cone. The plot is made with angular prescale parameter of $5^{\circ}$ and track length cut of $1.5 \mathrm{~m}$. . . . . . . . . . . . . . . . . 15 
2.9 Illustration of collecting rate within a $5^{\circ}$ circle: different positions on the sphere can give different number of bins within the cone. . . . . . 16

2.10 Plot of cosmic rate distribution versus angular proximity to the Moon direction. Plot made by L. Aliaga with cosmic pulser data. . . . . . . 18

2.11 Plot of cosmic rate distribution versus angular proximity to the Moon direction. Plot made with the Moon shadow trigger data. . . . . . . . 19

3.1 Dark Matter (DM) particles may annihilate in the core of the Sun producing a source of high-energy neutrinos . . . . . . . . . . . 21

3.2 Upward going trigger rate through time, each point is averaged rate through 5 runs of NOvA Far Detector. . . . . . . . . . . . . . . . 22

3.3 An example of fitting the electronics response curve to multiple readouts from a single cell hit. . . . . . . . . . . . . . . . . 23

3.4 Single-hit timing resolution as observed in NOA far detector data with fourpoint readout, before (a) and after (b) multi-point fine timing im-

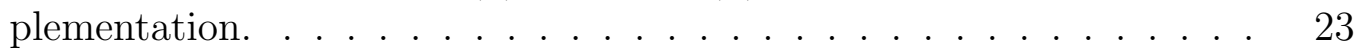

3.5 The expected versus observed time distribution for an upward-going muon track reconstructed in the NOvA far detector, using fine timing. The linear unconstrained fit (red solid line) has slope value close to 1. The fit with the upward-going track hypothesis ( $l o p e=1$ ) is shown as the blue-dashed line. The fit with the downward-going hypothesis is shown in the green dashed line and has a very poor probability. . 25

3.6 The slope distributions for cosmics (red) and WimpSim (blue) MC

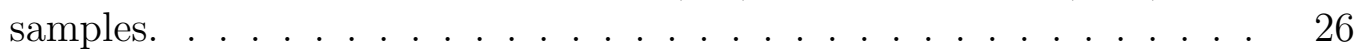

3.7 The LLR distributions for cosmics (red) and WimpSim (blue) MC samples. Note that only tracks longer then $5 \mathrm{~m}$ and with more than 50 hits are included. . . . . . . . . . . . . . . 26

3.8 A diagram of the various signal and control regions used in the analysis. 27

3.9 The azimuth and zenith angle of the Sun in the day (top) and in the night (bottom). . . . . . . . . . . . . . . . . 28

3.10 The $\cos (\Delta \theta)$ distribution for the day and the night samples. $\theta$ is the angular distance between the track and the solar position. Left plot shows initial distribution, right plot shows distribution with the Sun position being drawn randomly from the distribution that it follows at

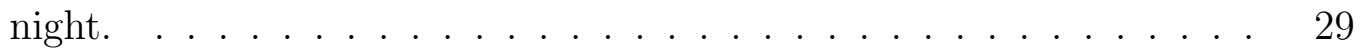

3.11 An example of an event that is prone to a possible misreconstruction. Two overlapping muon tracks with similar extent in the $z$-dimension and near coincidence in time (hits are colored by time). 2D track objects from each view must be merged to produce a full $3 \mathrm{D}$ object, but in this case it's ambiguous: is the correct matching (A1,B2) or $(\mathrm{A} 2, \mathrm{~B} 1) ? . \ldots \ldots \ldots \ldots$

3.12 An example of an event that is a good candidate for upward-going muon. The track (highlighted) passed all cuts and showed good probability to be going up. . . . . . . . . . . . . . . . . 31 
3.13 Distribution of the variables with respect to the elevation angle of the track, where 0 is horizontal, -1 is downward going, 1 upward going . 31

$4.1 \quad$ Example of visualization provided by EventDisplay module. . . . . 32

$4.2 \quad$ Example of visualization provided by DDT Event Display. . . . . . . 33

4.3 DDT Event Display visualization of tracks pointing to the Moon. Visualized data is recorded by the Moon trigger. . . . . . . . . . . . 36

4.4 DDT Event Display is used to compare tracks produced by the Moon $\begin{array}{ll}\text { shadow trigger before (grey tracks) and after (colored tracks) bug fix. } & 37\end{array}$

5.1 Sensitivity of different types of detectors (CSC and scintillators) to neutron background. . . . . . . . . . . . . . 38

5.2 Experimental setup for testing CSC: CSC, gas cylinder, movable stand, oscilloscope, cosmic telescope consisting of 2 scintillators . . . . . . . 39

5.3 First signal from cosmic particles registered with CSC . . . . . . . . . 41

5.4 Scheme of dependencies of NOvA software . . . . . . . . . . . . . . . 42

B.1 Fiducial volume of the Far Detector with $1 \mathrm{~m}$ cut. Values are in $\mathrm{m}^{3} . \quad 45$

B.2 Fiducial volume of the Far Detector with $10 \mathrm{~m}$ cut. Values are in $\mathrm{m}^{3} \quad 45$

B.3 Effective acceptance of the Far Detector with $1 \mathrm{~m}$ cut. Values are in $\mathrm{m}^{2} 46$

B.4 Effective acceptance of the Far Detector with $10 \mathrm{~m}$ cut. Values are in

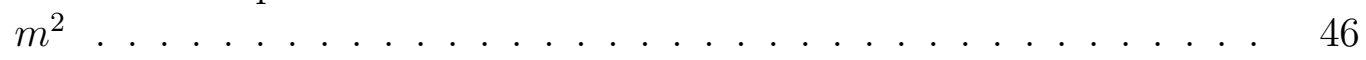

B.5 Track length in $\mathrm{cm}$ versus track number of hits in a track. The data is taken from cosmic pulser file. . . . . . . . . . . . . . . . . . . 47

C.1 Moon trigger rate plot taken from Ganglia . . . . . . . . . . . . . . . 48

C.2 Sun trigger rate plot taken from Ganglia . . . . . . . . . . . . . . . . 48

C.3 Sun and Moon trigger rate combined, separate plots are taken from Ganglia ......................... 49

F.1 Neutrino paths to NOvA Far Detector. . . . . . . . . . . . . . . . 55

F.2 Muon neutrino flux at NOvA FD depending of angle to horizon, values of angle in radians, values of flux are arbitrary . . . . . . . . . . . . . 56

F.3 The NOvA FD with surrounding rock . . . . . . . . . . . . . . 56

G.1 Example of duplicated tracks in the DDT system (duplicates are combined into groups and marked by the same color). Coordinates are given in cell and plane indices. . . . . . . . . . . . . . . . . 59

G.2 The DDT system efficiency VS track length (left) and time (right). The cosmic tracker is used for comparison with DDT. . . . . . . . . 59

G.3 The DDT system purity VS track length (left) and time (right). The cosmic tracker is used for comparison with DDT. . . . . . . . . . . 60

G.4 The DDT system purity including duplicate DDT tracks VS track length (left) and time (right). The cosmic tracker is used for comparison with the DDT. . . . . . . . . . . . . . 60 
G.5 Tracks in the offline event display for a cosmic pulser file, run 19429, subrun 32, event 480476. . . . . . . . . . . . . . . . . . 60

G.6 Tracks (DDT) in the 3D event display for cosmic a pulser file, run 19429, subrun 32, event 480476. 1: poorly reconstructed track . . . . 61

G.7 Tracks (cosmic tracker) in the 3D event display for a cosmic pulser file, run 19429, subrun 32, event 480476. 1,2,3: tracks are absent in the DDT 4: track is absent in both DDT and window tracker . . . . . . . 61

G.8 Tracks (window tracker) in the 3D event display for a cosmic pulser file, run 19429, subrun 32, event 480476. 1: track is absent in both DDT and cosmic tracker . . . . . . . . . . . . . . . . 62

H.1 Histogram of distribution of average number of hits per track by subrun of the NOvA Far Detector. Red line denotes a data quality cut on average number of hits. . . . . . . . . . . . . . . 63

H.2 Histogram of distribution of average track length by subrun of the NOvA Far Detector. Red line denotes a data quality cut on average track length. . . . . . . . . . . . . . . . 63

H.3 Histogram of distribution of average track $\beta$ by subrun of the NOvA $\begin{array}{ll}\text { Far Detector. Red line denotes a data quality cut on average track } \beta . & 64\end{array}$

J.1 Parts of the DDT Event Display: part 1 . . . . . . . . . . . . . . . . 68

J.2 $\quad$ Parts of the DDT Event Display: part 2 . . . . . . . . . . . . . 68

J.3 The DDT Event Display with a light theme . . . . . . . . . . . . . . 69

J.4 How to use the DDT Event Display: rotate . . . . . . . . . . . . . . . 69

J.5 How to use the DDT Event Display: move . . . . . . . . . . . . . . . 70

J.6 How to use the DDT Event Display: zoom . . . . . . . . . . . . . . . 70

K.1 The geometry of a Cathode Strip Chamber produced for Mu2e experiment. . . . . . . . . . . . . . . . . 71

K.2 The CSC circuit scheme . . . . . . . . . . . . . . . . . . . . 72

K.3 The CSC cathode connectors . . . . . . . . . . . . . . . . . . . . . 72

K.4 One layer of an opened CSC . . . . . . . . . . . . . . . . . . . . . 72

L.1 The scheme used for CSC noise measurements . . . . . . . . . . . . . 73

L.2 The noise (in $\mathrm{Hz}$ ) of produced cathode strip chambers VS applied voltage 74

L.3 The scheme used for the CSC noise measurements . . . . . . . . . . . 75

L.4 The efficiencies of produced cathode strip chambers VS applied voltage 75

L.5 $\quad$ Scheme used for the CSC signal amplitude measurements . . . . . . . 76

L.6 Signal amplitude of selected cathode strip chamber (CSC3) VS applied 7 


\section{List of ABBREviATions}

DOE US Department Of Energy

Fermilab Fermi National Accelerator Laboratory. IL, USA

NOvA NuMI off-axis $\nu_{e}$ appearance experiment

DDT Data Driven Trigger system of NOvA

DAQ Data Acquisition system of NOvA

FD NOvA Far Detector

ND NOvA Near Detector

PDG Particle Data Group

PMNS Pontecorvo-Maki-Nakagawa-Sakata matrix of neutrino flavor mixing

MSV Michaev-Smirnov-Wolfenstein matter effect

NH, RH Normal Hierarchy of neutrino masses and Reversed Hierarchy respectively

Mu2e Muon to electron neutrinoless conversion experiment

BNL Brookhaven National Laboratory. NY, USA

JINR Joint Institute of Nuclear Research. Dubna, Russia

CLFV Charged Lepton Flavor Violation

CRV Cosmic Ray Veto system for Mu2e

NOVAS Naval Observatory Vector Astrometry Software

DM Dark Matter

WIMP Weakly Interacting Massive Particle - candidate for DM particle

ADC Amplitude to Digital Converter, also used as general unit of amplitude

TDC Time to Digital Converter, also used as general unit of time HV High Voltage 


\section{CHAPTER 1 INTRODUCTION}

This thesis describes the work I provided for UVA while working under the supervision of Prof. C. Group. The bulk of this work was completed during my stay at Fermi National Accelerator Laboratory (Fermilab, IL, USA [1]) from May 2015 to November 2016.

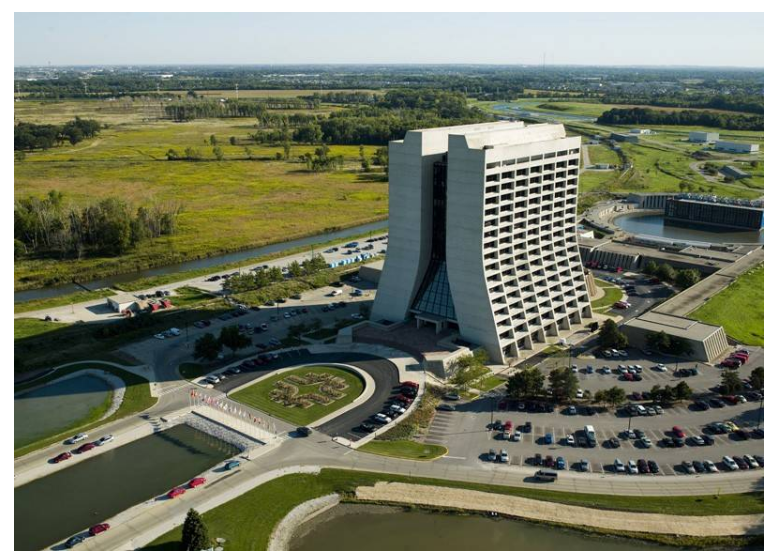

Figure 1.1: Fermi National Accelerator Laboratory

One of the features of research in experimental particle physics is that the lifetime of experiments is in many cases longer than the time people spend in graduate school or in a postdoc contract, so, some research projects are finished, and others will necessarily remain unfinished and will be continued by the next generation of graduate students. In this regard, experimental physics is closer to industry than to theoretical physics.

Another feature of experimental physics is that most of the students are involved in several research topics and provide technical services for the experiment, which plays an important part for the experiment's success. So, to fully describe the work I've done, I need to split this thesis into several sections which are only loosely coupled to each other.

My main field of activity at Fermilab was work on the Data-Driven Trigger (DDT) systems for the neutrino experiment NOvA, and this thesis will be mainly dedicated to this work. The DDT is a set of software which analyses data as it comes from the Far Detector (FD) or Near Detector (ND) and records events matching some specific criteria - such as specific track directions, lengths, deposited energy etc. It includes making software algorithms, providing support and tests to ensure it runs correctly, 
providing data quality checks and data analysis, and also managing the builds for the DDT system as a whole.

I also worked on an experiment held in Fermilab called Mu2e - there I was mainly involved in fabrication of Cathode Strip Chambers (CSC) for Cosmic Ray Veto (CRV) system of this experiment.

The NOvA and Mu2e experiments and my work on these experiments will be described in more details below.

\section{$1.1 \quad$ NOvA}

NOvA [2] is an electron neutrino appearance experiment based at Fermilab. It's main goal is to measure parameters related to neutrino oscillations. The experiment uses the NuMI neutrino beam (Neutrinos at the Main Injector) and two detectors, one of which - Near Detector (ND) - is located 300 feet (100 meters) below the surface in Fermilab, IL, and another one - Far Detector (FD) - is placed on the surface 810 km (500 miles) away, in Ash River, MN (see Fig. 1.2).

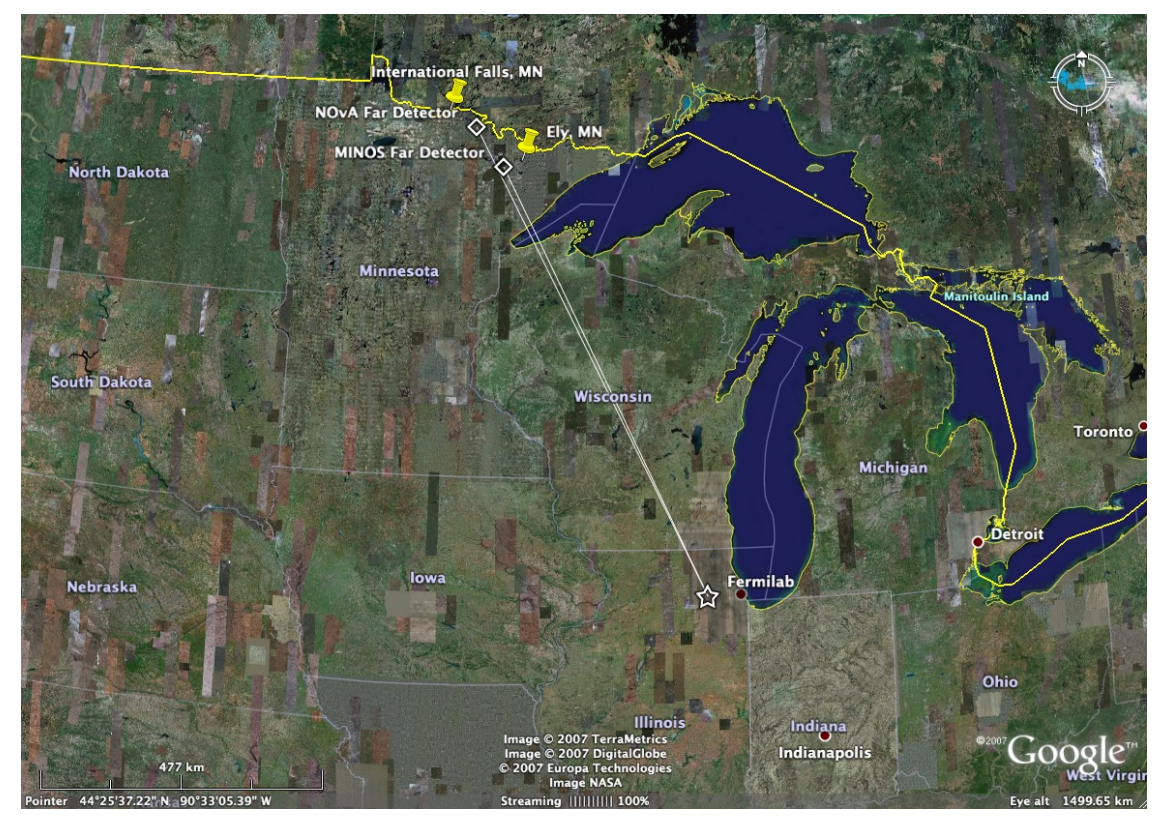

Figure 1.2: Location of Fermilab and NOvA Far Detector

The NuMI beam produces muon neutrinos (switch to muon antineutrino beam is planned when the experiment collects enough statistics with neutrino events) which then oscillate to another neutrino flavor while moving through the Earth. The main mode of oscillation that will be studied with NuMI neutrino energy and the NOvA length of baseline ( $2 \mathrm{GeV}$ mean neutrino energy and $810 \mathrm{~km}$ baseline) is the oscillation to electron neutrino. Studying this conversion and the related disappearance of muon neutrinos, as well as measuring some other neutrino properties are the main goals of the experiment. 
To be more specific, let's highlight the theory of neutrino oscillations and then name parameters that NOvA can potentially measure with higher precision than other experiments.

There are several mysteries which lead to large interest to neutrino physics. Neutrinos demonstrate features which are not well described by the SM - they violate flavor symmetry, performing oscillations while they propagate. These oscillations are described by a mismatch in the flavor and energy eigenvectors, so neutrinos in some definite energy state, are a superposition of 3 flavor states. The matrix of transformation from the mass eigenstate basis to the flavor eigenstate basis is called PontecorvoMaki-Nakagawa-Sakata matrix (PNMS) and usually is parameterized with 3 mixing angles and $1 \mathrm{CP}$-violation phase (so called Dirac phase).

$$
\left|\nu_{i}\right\rangle=U_{\alpha i}\left|\nu_{\alpha}\right\rangle
$$

where $i$ is mass index, $\alpha$ is flavor index and matrix $U_{\alpha i}$ is

$$
U_{\alpha i}=\left[\begin{array}{ccc}
c_{12} c_{13} & s_{12} c_{13} & s_{13} e^{-i \delta_{C P}} \\
-s_{12} c_{23}-c_{12} c_{23} s_{13} e^{i \delta_{C P}} & c_{12} c_{23}-s_{12} s_{23} s_{13} e^{i \delta_{C P}} & s_{23} c_{13} \\
s_{12} s_{23}-c_{12} c_{23} s_{13} e^{i \delta_{C P}} & -c_{12} s_{23}-s_{12} c_{23} s_{13} e^{i \delta_{C P}} & c_{23} c_{13}
\end{array}\right]
$$

Here $c_{i j}$ and $s_{i j}$ stand for $\cos \theta_{i j}$ and $\sin \theta_{i j}$ respectively, where $\theta_{i j}$ are neutrino mixing angles. $\delta_{C P}$ is the Dirac CP violation phase, which is an important parameter to study since it may be an important part in forming the particle-antiparticle asymmetry in early Universe, thus playing a role in the matter dominance over antimatter in the Universe. The value of this parameter is undetermined.

There are two competing alternatives on the nature of neutrino mass: the mass term in SM Lagrangian can take either Dirac (1.3) or Majorana (1.4) form.

$$
\begin{aligned}
& \mathcal{L}_{m}^{D}=-m \psi_{R}^{\dagger} \psi_{L} \\
& \mathcal{L}_{m}^{M}=m \psi_{L}^{T} \sigma_{2} \psi_{L}
\end{aligned}
$$

Here $\sigma_{i}$ are Pauli matrices and $\psi_{L, R}=\frac{1}{2}\left(1 \pm \gamma_{5}\right) \psi$ - left and right spinors.

In case of a Majorana mass term, the PMNS matrix can be supplied with 2 additional CP violating phases $\alpha_{21}$ and $\alpha_{31}$, then it takes form (1.5).

$$
\begin{aligned}
& U_{\alpha i}= \\
& {\left[\begin{array}{ccc}
c_{12} c_{13} & s_{12} c_{13} & s_{13} e^{-i \delta_{C P}} \\
-s_{12} c_{23}-c_{12} c_{23} s_{13} e^{i \delta_{C P}} & c_{12} c_{23}-s_{12} s_{23} s_{13} e^{i \delta_{C P}} & s_{23} c_{13} \\
s_{12} s_{23}-c_{12} c_{23} s_{13} e^{i \delta_{C P}} & -c_{12} s_{23}-s_{12} c_{23} s_{13} e^{i \delta_{C P}} & c_{23} c_{13}
\end{array}\right] \times\left[\begin{array}{ccc}
1 & 0 & 0 \\
0 & e^{\alpha_{21} / 2} & 0 \\
0 & 0 & e^{\alpha_{31} / 2}
\end{array}\right]}
\end{aligned}
$$

One consequence of this mechanism of flavor symmetry violation is that neutrinos appear to have masses whilst the SM describes them as massless particles. Moreover, neutrino masses are not yet measured to any precision, but an upper limit on the neutrino mass is set much lower than any other known particle mass $(<0.3 \mathrm{eV}$, which is 
about $10^{6}$ times lighter than next lightest particle - electron). The mass difference between different neutrino mass states is measured using data from neutrino oscillation experiments like NOvA $\left(\Delta m_{12}^{2} \approx 7.5 \times 10^{-5} \mathrm{eV}^{2}, \Delta m_{13}^{2, N H} \approx 2.4 \times 10^{-3} \mathrm{eV}^{2}\right)$.

Although we are able to measure neutrino mass differences, the order of the mass states remains unknown. In other words it's not known if state 1 and state 2 are less massive than state 3 (normal mass hierarchy or $\mathrm{NH}$ ) or more massive than state 3 (reversed mass hierarchy or RH). It's natural to assume the normal mass hierarchy since neutrino mass states 1 and 2 include larger contribution of electron neutrino, while state 3 is almost equal mixture of muon neutrino tau neutrino, and the masses of all known particles are increasing with generations. But so far no evidence for either normal or reversed hierarchy has been provided by any experiment. See Fig. 1.3 for an illustration of the neutrino mass hierarchy.

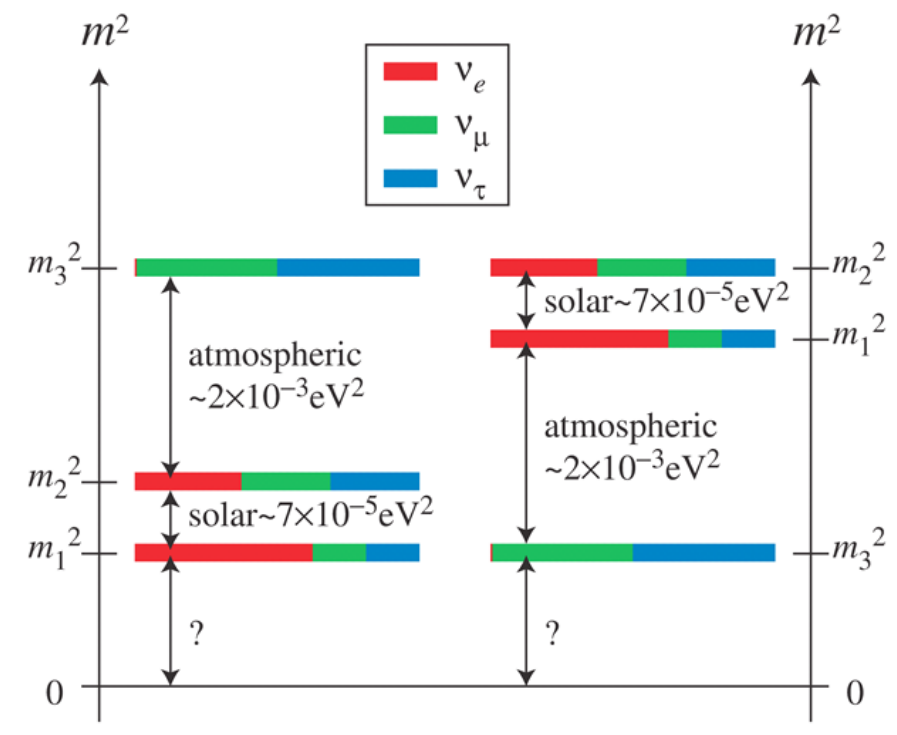

Figure 1.3: Neutrino mass hierarchy.

Among the parameters mentioned in the formulas above, NOvA can make advances in measuring these:

- Mixing angle $\theta_{13}$

- Dirac phase of $\mathrm{CP}$ violation

- Normal/reversed mass hierarchy

For completeness, I'll also include here formulas for probability of neutrino flavor change. If there were only 2 neutrino flavors (say, $\nu_{\mu}$ and $\nu_{e}$ ), the probability of 
changing flavor would be given by the following formula.

$$
\begin{aligned}
& P_{\nu_{\mu} \rightarrow \nu_{e}}=\sin ^{2}(2 \theta) \sin ^{2}\left(\frac{\Delta m^{2} c^{3} L}{4 \hbar E}\right)= \\
& \sin ^{2}(2 \theta) \sin ^{2}\left(1.27 \frac{\Delta m^{2}\left(e V^{2}\right) L(k m)}{E(G e V)}\right)
\end{aligned}
$$

Here $L$ is the distance the neutrino travels and $E$ is the neutrino energy.

With the more realistic assumption of 3 flavors and taking into account the Michaev-Smirnov-Wolfenstein (MSW) matter effect, the probability of conversion from $\nu_{\mu}$ to $\nu_{e}$ is modified as follows:

$$
\begin{aligned}
& P_{\nu_{\mu} \rightarrow \nu_{e}}=\sin ^{2}\left(2 \theta_{13}\right) \sin ^{2}\left(\theta_{23}\right) \frac{\sin ^{2}[(A-1) \Delta]}{(A-1)^{2}}+ \\
& 2 \alpha \sin \left(\theta_{13}\right) \sin \left(2 \theta_{12}\right) \sin \left(2 \theta_{23}\right) \cos \left(\delta_{C P}+\Delta\right) \frac{\sin [A \Delta] \sin [(A-1) \Delta]}{A(A-1)}
\end{aligned}
$$

Here $\alpha=\frac{\Delta m_{12}^{2}}{\Delta m_{13}^{2}}, \Delta=\frac{\Delta m_{13}^{2} L}{4 E}, A= \pm \frac{G_{f} n_{e} L}{\sqrt{2} \Delta}(+\operatorname{sign}$ for $\nu,-\operatorname{sign}$ for $\bar{\nu}), n_{e}$ is electron density in matter and $G_{f} \approx 1.17 \times 10^{-5} \mathrm{GeV}^{-2}$ is Fermi interaction constant.

The latest estimates for neutrino oscillation parameters measured by a variety of experiments are shown in Appendix A.

As mentioned above, NOvA has two detectors, one of which is located at Fermilab (ND) and the second one located in North Minnesota (FD). The ND has $0.3 \mathrm{kT}$ of active detector mass and located underground, which provides shielding from cosmic rays. The FD weights $14 \mathrm{kT}$ and is located on the surface, thus exposed to cosmic rays, which makes a background for oscillation studies of beam neutrinos, but at the same time provides a good source of cosmic data, which allows calibration and other studies at NOvA such as searches for supernova bursts, study of cosmic showers, exotic particles etc.

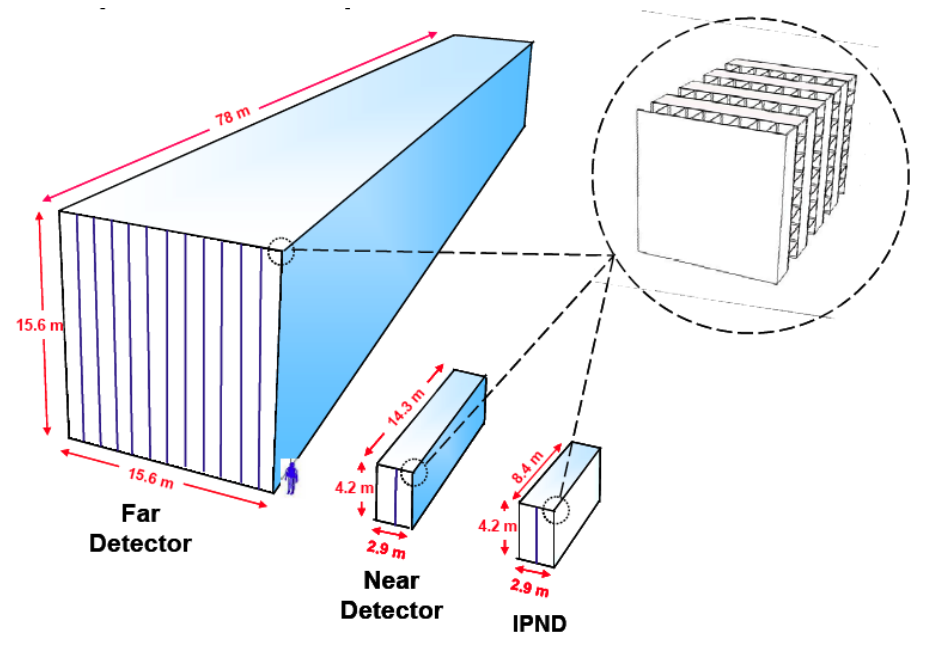

Figure 1.4: The NOvA Near and Far Detectors 
Internally, the FD and $\mathrm{ND}$ are constructed in a similar way: both consist of plastic extrusions (PVC) of $4 \times 6 \mathrm{~cm}^{2}$ cross section filled with liquid scintillator and wavelength shifting fibers (WLS) which end at avalanche photodiodes (APD) - see Fig.1.5. These elements form a cell in the detector. A passing charged particle disturbs the media and it emits light which then propagates in fibers and is transformed into electric signals at the photodiodes. Layers of orthogonal extrusions form pictures of particle tracks in 2 views ( $\mathrm{XZ}$ view and $\mathrm{YZ}$ view, where $\mathrm{Z}$ is the axis aligned with direction of the beam, $\mathrm{Y}$ axis points vertically up, and $\mathrm{X}$ is orthogonal to these two) - see Fig.1.6.

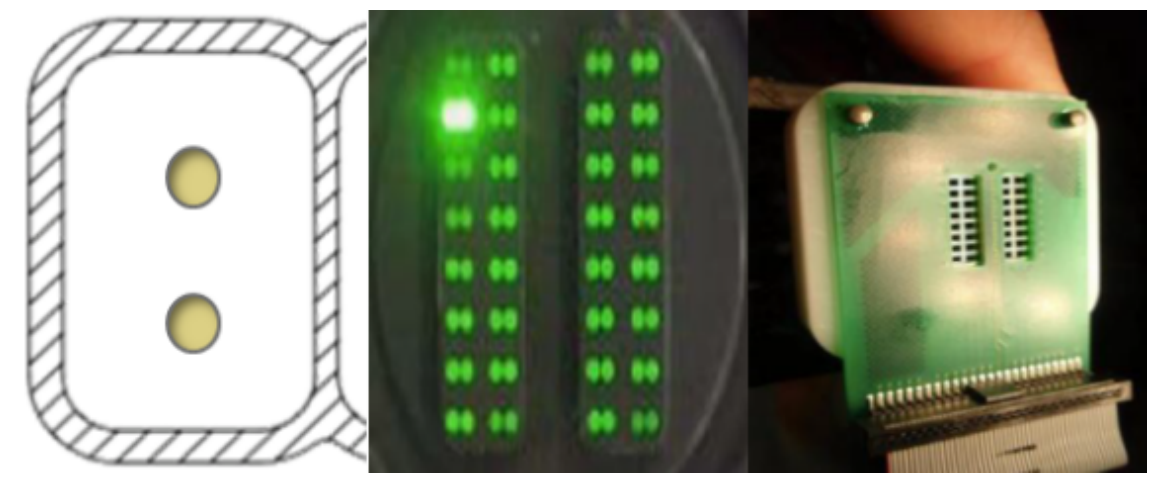

Figure 1.5: From left to right: PVC, fibers and avalanche photodiodes which form cells of NOvA detectors

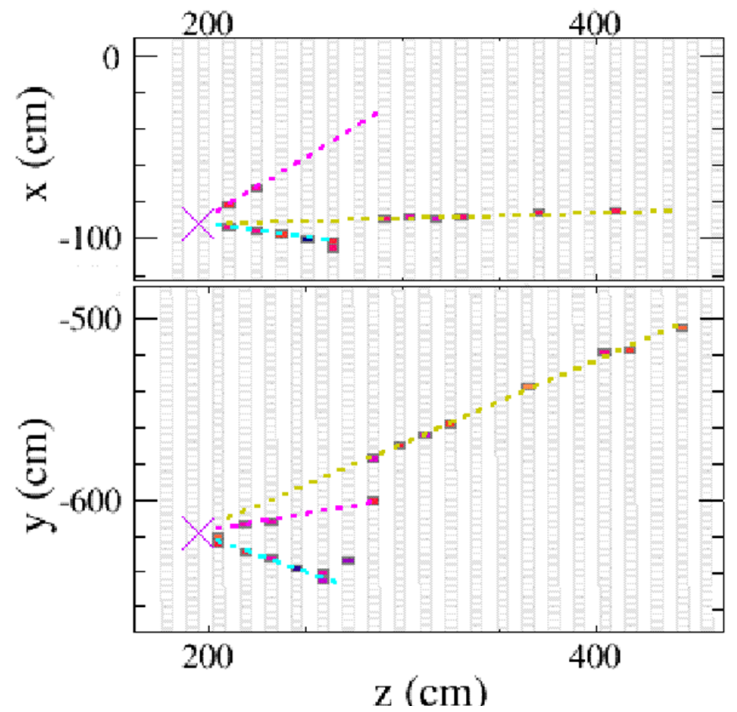

Figure 1.6: First neutrino candidate in 2 views of the NOvA FD event display

Cell signals (hits) are collected by the NOvA data acquisition system (DAQ) with the use of software data-driven triggers (DDT), which send messages telling the DAQ to collect some specific chunk of data if an event with some required features 
happened. The collected data is then analysed using offline analysis software, based on an event processing framework called ART [3] and a large scale data analysis framework called ROOT [4].

The UVA group is supervised by Prof. C. Group and Prof. C. Dukes and is mainly involved in exotic searches using the NOvA detectors. These are searches of monopoles, upward-going muons and studies of the Moon shadow in cosmic rays.

\section{$1.2 \mathrm{Mu} 2 \mathrm{e}$}

Mu2e [5] is an experiment held at Fermilab that should shed light on the possibility of neutrinoless conversion from a muon into an electron and thus charged lepton flavor violation (CLFV) - see Fig. 1.7. This type of conversion is highly suppressed in the Standard Model (SM), so any events of this type will indicate a direct violation of the Standard Model and the existence of new physics beyond it.
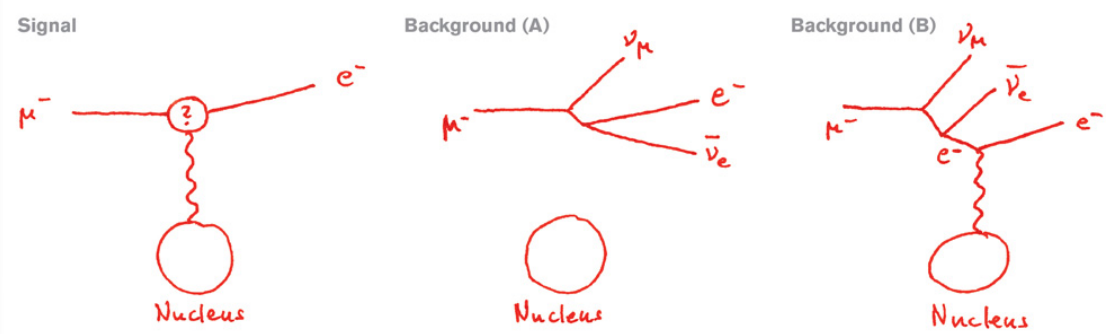

Figure 1.7: Signal and background processes for the Mu2e experiment

The experiment is designed to produce a beam of muons which hit an aluminum target and may form muonic atoms - atoms with one of the electrons substituted with a muon. Since muons have sufficiently higher mass than the electron, eventually, muons captured by atomic nuclei will decay forming an electron and two neutrinos. In this conventional decay, neutrinos take away some of the energy while in the case of neutrinoless conversion all of the energy goes to the electron. So in this hypothetical exotic mode of decay, the electrons will have an energy of $\sim 105 \mathrm{MeV}$, which corresponds to the muon mass - see Fig. 1.8.

The Mu2e apparatus is formed of a tungsten target which is hit by the beam of $8 \mathrm{GeV}$ protons coming from the Fermilab Delivery ring (producing pions which decay into muons), an s-shaped solenoid which selects low-energetic muons, an aluminum target which gets hit by the low-energy muon beam, and a detector consisting of a magnetic spectrometer for measuring the momentum of the particles and an electromagnetic calorimeter. Fig. 1.9 depicts the Mu2e design.

Registering events with the particular electron energy of $105 \mathrm{MeV}$ will indicate the emergence of the exotic process and thus the violation of the SM. The main challenge that Mu2e is exposed to is removing background processes which can mock the $105 \mathrm{MeV}$ electrons and thus produce a false signal. The main sources of background 


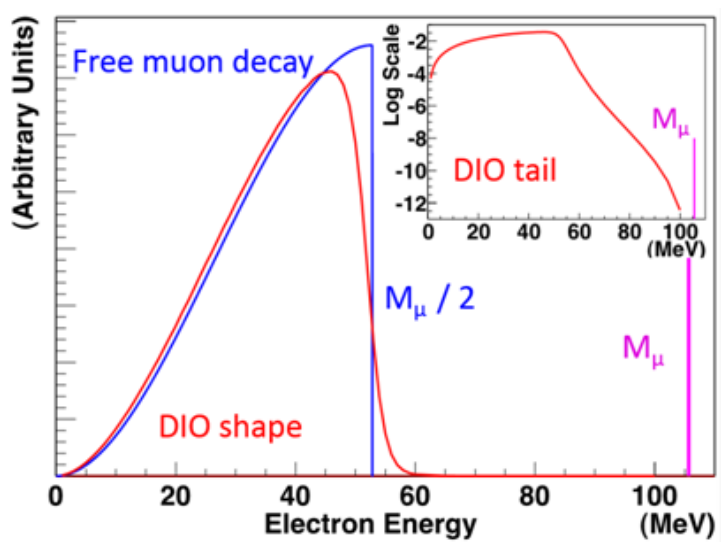

Figure 1.8: The expected spectrum of electron energies at the Mu2e detector. DIO stands for the decay in orbit process

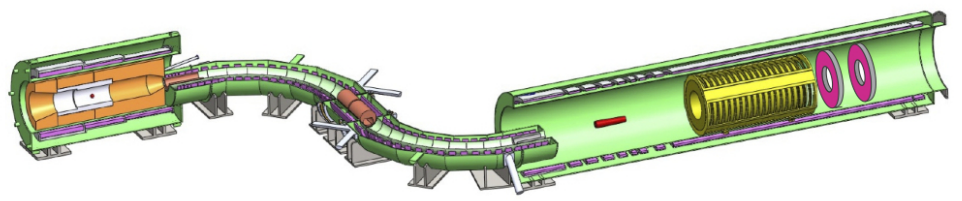

Figure 1.9: Design of the Mu2e experiment

are tail of the muon-to-electron decay-in-orbit distribution and secondary particles from cosmic rays.

To reduce the background emerging from cosmic particles, both passive and active shielding will be used. Active shielding means detection of cosmic particles which can mock the signal and spoil the experimental data. The active shielding is called the Cosmic Ray Veto (CRV). It will consist of 4 layers of scintillator panels with aluminum absorbers between each layer - see Fig. 1.10. Panels are overlapped with each other to cover all exposure area and to register all coming particles. The requested efficiency of the CRV system is $99.99 \%$, thus for a three-out-of-four coincidence requirement each layer of scintillators is required to show efficiency of $99.6 \%$. Overall, the number of background events (after shielding) is expected to be 0.4 for 3-year run.

UVA leads the efforts to design, test and fabricate the CRV system. In order to assure that scintillators meet efficiency requirements, their efficiency will be measured using cosmic ray muons, which involves using two cathode strip chambers (CSC's), each containing several layers. The chambers were designed by Brookhaven National Laboratory (BNL) and fabrication involved work by Fermilab, UVA and JINR specialists. 


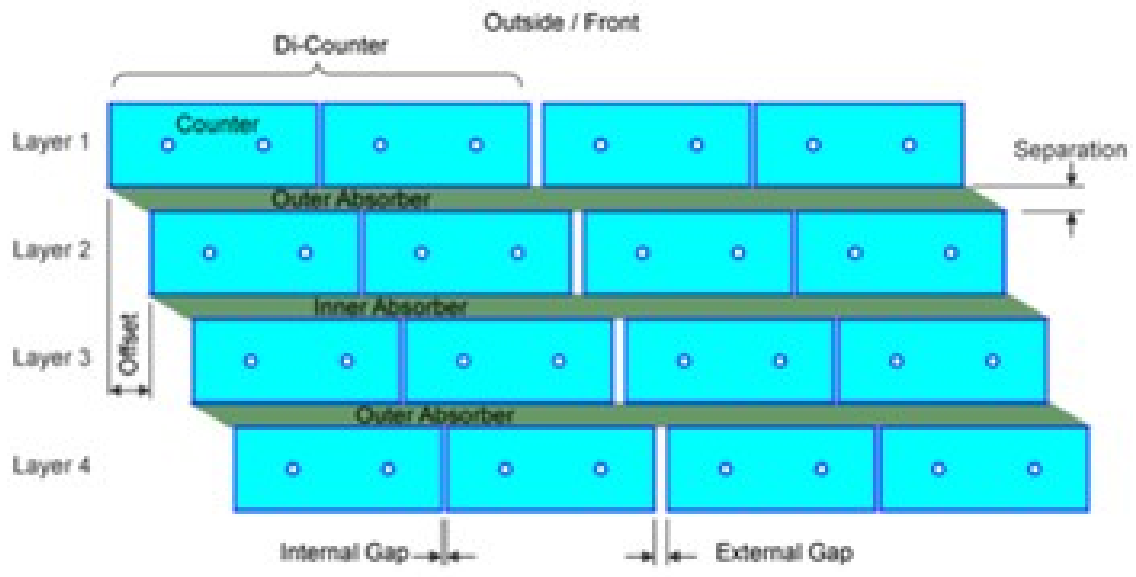

Figure 1.10: 4 layers of scintillators forming active shielding system (CRV) for Mu2e experiment 


\section{CHApter 2 Moon Shadow Studies}

\subsection{Motivation}

Understanding the angular resolution of the NOvA Far Detector is important in any studies sensitive to the direction of particle tracks. We can measure the angular resolution by looking at signatures in the particle flux from objects with well defined coordinates. One option is to use cosmic rays to study objects and their shadows in a similar way to how we might see them using visual light (photons). For this to happen we can look at the closest celestial bodies to the Earth - the Moon and Sun - and see how they look using cosmic ray muons.

What we expect to see is the shadow that the Sun or the Moon creates in the flux of cosmic particles. Similar studies were recently done for the MINOS experiment [8], where they studied the cosmic ray shadow caused by the the Moon. The shape and position of the shadow is determined by many factors including the angular resolution of the NOvA Far Detector and the influence of magnetic fields acting on charged particles.

A preliminary analysis was conducted by Caltech postdoc Chris Backhouse and showed the existence of the Moon shadow using the NOvA Far Detector [9, 10]. The data sets used to conduct this analysis was based on the unbiased cosmic pulser data. Cosmic pulser is a software recording $550 \mu \mathrm{s}$ time windows of cosmic activity with a frequency of $10 \mathrm{~Hz}$. The total number of events contained in this dataset is $\approx 10^{8}$. The resulting plot showing evidence of the shadow from the Moon (by shadow one should understand here a lower rate of cosmic particles) is shown on Fig. 2.1.

The deficiency is fitted with a Gaussian function and is seen with a statistical significance of about $4 \sigma$, but with a poor $\chi^{2} / \#$ d.o.f ratio $(100.7 / 48)$. In order to study the Moon shadow with less uncertainty we need to collect more data.

\subsection{DDT Moon and Sun trigger}

In order to collect more data, a data-driven Moon/Sun software trigger was introduced as a part of the NOvA DDT system. The trigger went live on the Far Detector on Aug 11th 2015. To check the validity of the algorithm several sets of cosmic pulser files were used, each set contained 24 files (one taken each hour of a day). It was shown with this check that the Moon trigger has a reasonable pattern of acquired rates following the position of the Moon in the sky. When the Moon is below the 


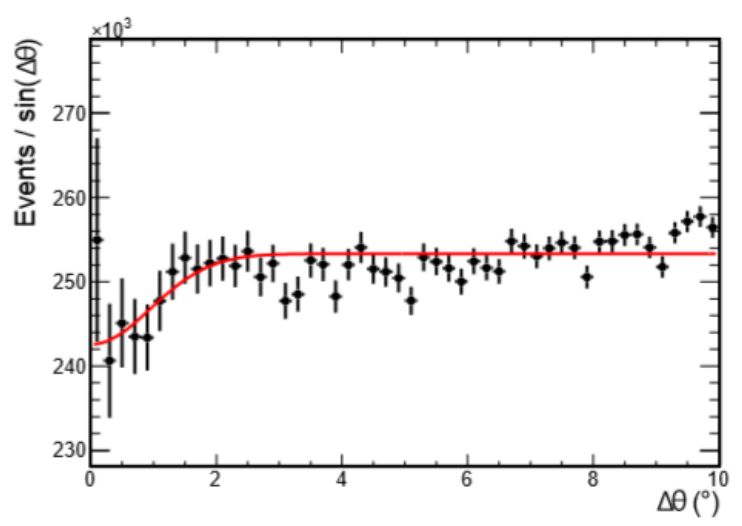

Figure 2.1: Preliminary Moon shadow analysis done by C. Backhouse. The deficit in the cosmic particles rate shows shadowing of particle flux by the Moon.

horizon, the trigger doesn't record any events, and it starts recording when the Moon rises above the horizon - see Fig. 2.2, 2.3 .

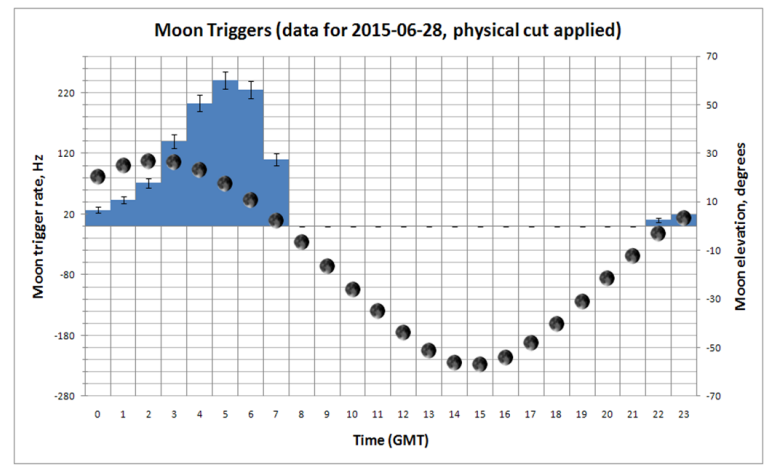

Figure 2.2: Rate of cosmic particle tracks triggered by Moon trigger with cut for false tracks. The Moon elevation is marked by dark circles. Data taken from cosmic pulser files of Jun 282015

\subsection{Acceptance and fiducial volume}

Plots of the trigger rates don't fully correlate with the Moon/Sun elevation angles. We observe a time shift between the maximal elevation of Sun or Moon and the maximal registered cosmic rate. It seems natural that these maxima should coincide, since the rate of cosmic muons with energies of several $\mathrm{GeV}$ (which form majority of registered particles) decreases with zenith angle approximately as $\cos ^{2}(\theta)$ [11, and so the maximal rate is expected to correspond to maximal elevation. Plots of trigger rates made for different datasets confirms that the shift exists, but that the offset of the peak rates and the Sun/Moon elevation varies for each dataset.

In order to understand this feature of the cosmic rates, the angular distribution of cosmic rates was studied. The plot of the cosmic angular distribution for the case of 


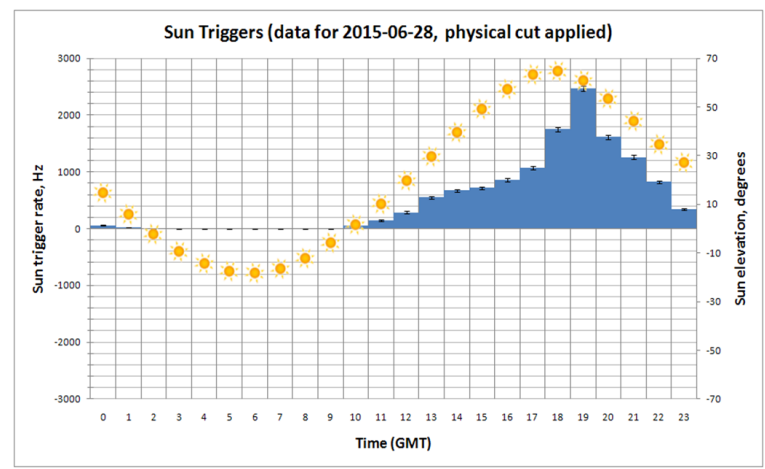

Figure 2.3: Rate of cosmic particle tracks triggered by Sun trigger with cut for false tracks. The Sun elevation is marked by yellow circles. Data taken from cosmic pulser files of Jun 282015

$3 m$ track length requirement is shown in Fig. 2.4. After an analysis of rates collected from different angles, the main source of this discrepancy was found to be related to the Far Detector geometry.

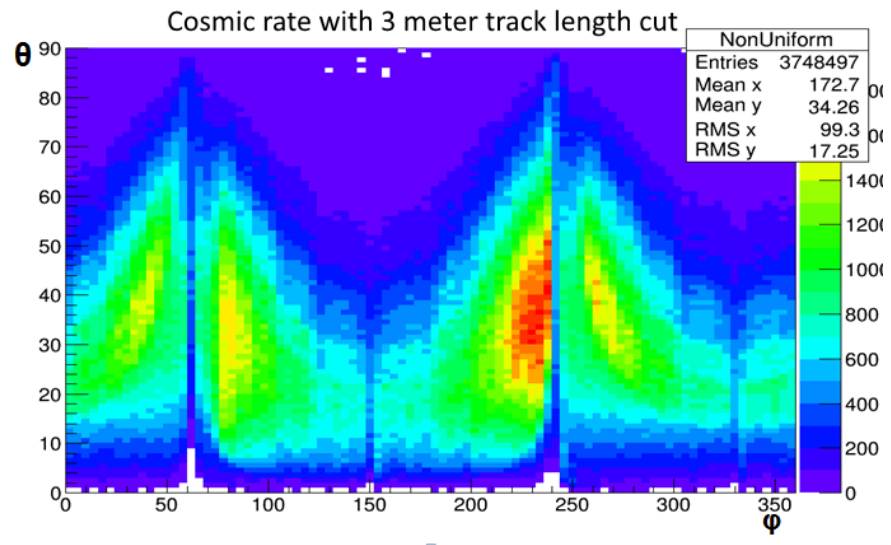

Figure 2.4: Cosmic rate distribution in NOvA Far Detector

This plot shows a large nonuniformity of the cosmic rate in both azimuthal and zenith angles. Furthermore, when one studies the Moon/Sun positions overlaid with plots of the cosmic rate one can make a convincing explanation of why we have an apparent shift between the maximal elevation and maximal cosmic rate. The source of the non-uniformity is related to the geometry of the detector (it's seen from plots above, since the direction of rate decrease coincides with the detector's position to North).

The fiducial volume of the detector and its acceptance effectively depend on the track length requirement and incident angle of the incoming particle. Say, $10 \mathrm{~m}$ tracks with $45^{\circ}$ zenith angle cannot be found in the corners of the detector, so these corners should not be included in the fiducial volume of the detector. For the visual explanation of this statement see Fig. 2.5, 2.6.

Using a simple model of cosmic particle appearance in the detector and applying 


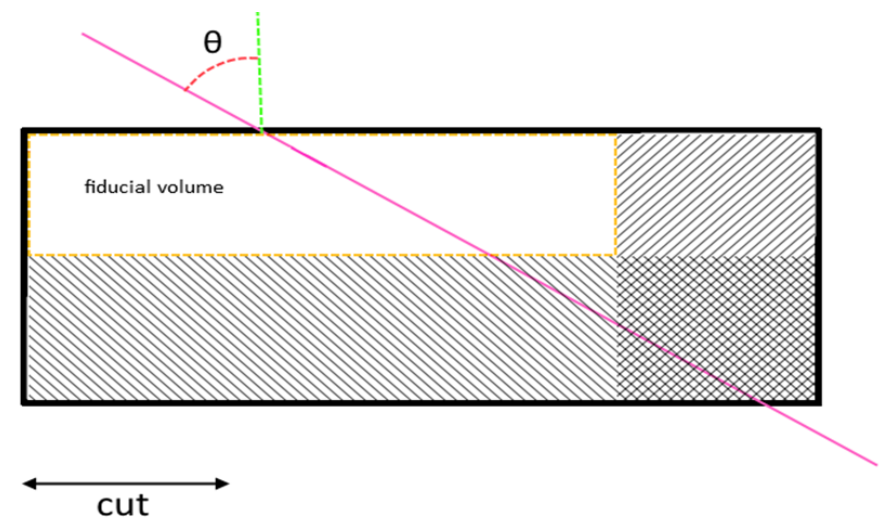

Figure 2.5: The scheme for the calculation of fiducial volume of the Far Detector. Only volume capable of producing registered particle with given length cut is included as fiducial. Volume by sides of the detector is not active.

a simple fiducial volume cut, the plots of the expected particle flux vs azimuth and zenith were made. We can check if this model explains the nonuniformity observed in the cosmic ray rate in the NOvA Far Detector.

Majority of cosmic particles are born outside of the detector, so, ignoring the contained-vertex events, the acceptance determines the rate of cosmic tracks. The acceptance multiplied by $\cos (\theta)^{2}$, where $\theta$ is zenith angle, should be proportional to the rate of registered tracks. Here $\cos (\theta)^{2}$ describes well the cosmic rate dependence on zenith angle for several $\mathrm{GeV}$ muons. Indeed the plot of this value (with cut of 1 meter implied), shown on Fig. 2.7 has a structure similar to the one observed in the data in Fig. 2.4.

More plots based on the acceptance and fiducial volume of the NOvA Far Detector are shown in Appendix B.

\subsection{Smart prescale}

The Moon trigger was introduced as part of the NOvA DDT system to collect more data than is available from the cosmic pulser files. Thus the trigger algorithm should be optimized to collect as much data as possible. The rate of muons pointing near the Sun or the Moon is too high for the capabilities of the DAQ system, so a prescale strategy must be selected to optimize data collection.

The trigger is designed to collect data within a $5^{\circ}$ cone around the celestial object - the Moon or the Sun. The expected collected cosmic rate was studied in order to determine the best possible prescale values - see Fig. 2.8.

According to this plot we expect to see a peak of the trigger rate at $\approx 2000 \mathrm{~Hz}$ daily. The DAQ systems we use can afford trigger rates of $\approx 20 \mathrm{~Hz}$, so the simplest choice of a prescale factor would be 100. But most of the time the rates are much lower, so using this as a flat prescale factor would reject far more data than required by the system. 


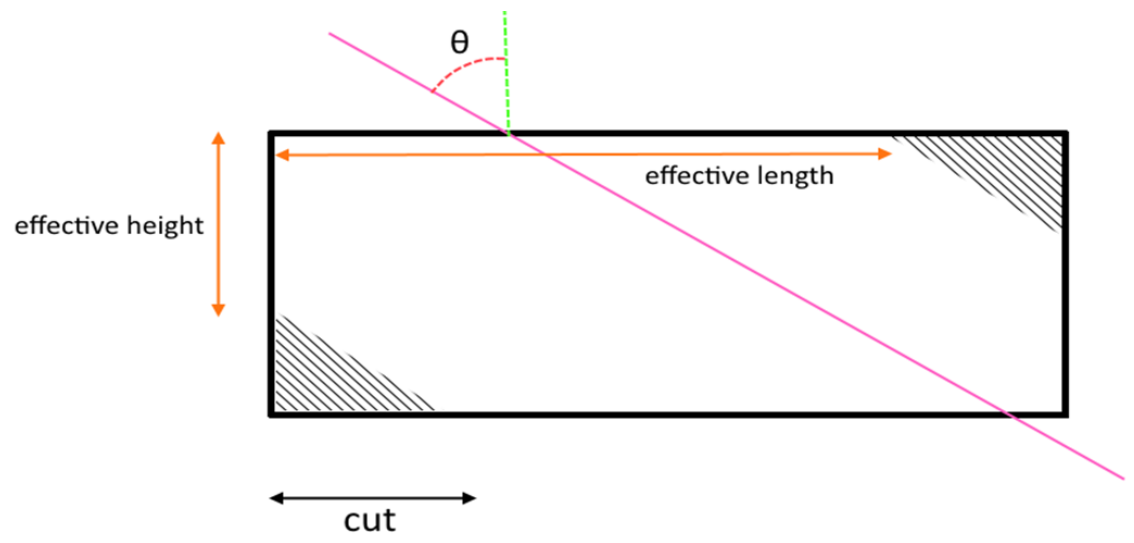

Figure 2.6: The cheme for calculation of effective acceptance of the Far Detector. Only surface capable of producing registered particle with given length cut is included as effective. Surface by corners of the detector is not active.

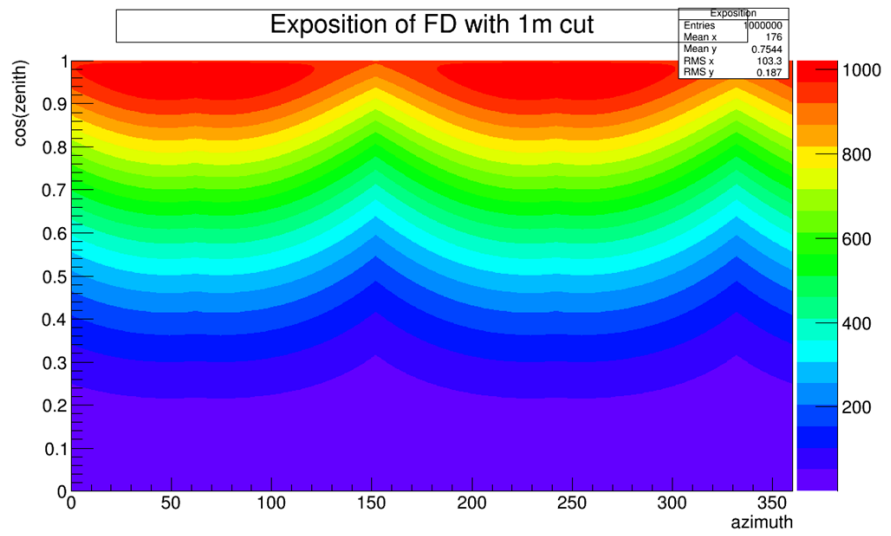

Figure 2.7: Acceptance of the Far Detector with $1 \mathrm{~m}$ cut multiplied by factor of $\cos ^{2}(\theta)$ from zenith angle flux dependence. Values in $m^{2}$

Another possible choice would be the use of cosmic pulser files for the analysis. As said above, cosmic pulser collects data with a $10 \mathrm{~Hz}$ frequency and uses a time window of $550 \mu \mathrm{s}$. So, overall with the cosmic pulser we read $1 / 200$ of incoming cosmic flux, which corresponds to a prescale of 200. A third possibility is to make a dynamic prescale (we call it "smart prescale") which will reduce the data to $20 \mathrm{~Hz}$ adjusting the rate at every position of Sun/Moon in the sky. Assuming that Sun/Moon rates grow and fall linearly during the day, it will give us 2 times more collected events than if we statically prescale to the maximal daily rate. So, this will give effective prescale of $\approx 50$. Note that since the smart prescale is a probabilistic algorithm, the rates can differ for identical data samples.

With all that said, the total estimated collected rate for tracks within a $5^{\circ}$ of the Sun/Moon in 1 year of running (assuming 100\% of livetime):

- Static prescale 100 : $\approx 1.5 \times 10^{8}$ tracks 


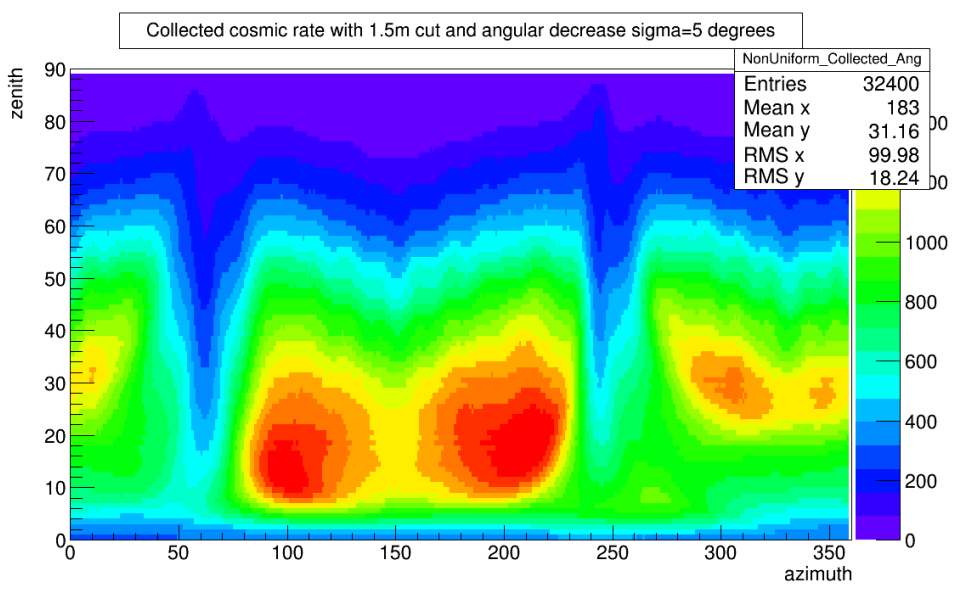

Figure 2.8: The expected rate of cosmics collected by the Moon trigger within a $5^{\circ}$ cone. The plot is made with angular prescale parameter of $5^{\circ}$ and track length cut of $1.5 \mathrm{~m}$

- Cosmic pulser: $\approx 0.75 \times 10^{8}$ tracks

- Smart prescale: $\approx 3 \times 10^{8}$ tracks

In addition to restricting the number of triggered tracks, a mechanism of "angular suppression" was introduced. This is a mechanism which allows the algorithm to trigger on tracks which are closer to true Moon/Sun direction with higher probability. This mechanism along with the smart prescale makes triggering probabilistic, and parameters used for the trigger are set to make the average trigger rate $20 \mathrm{~Hz}$. For a particular position of the Sun/Moon it can be larger or lower with expected uncertainty of $\approx \sqrt{20} \mathrm{~Hz} \approx 4-5 \mathrm{~Hz}$, which is acceptable value for DAQ system. The following function is used for angular suppression - that is, this function, taken as a multiplier for the probability of triggering a particle, defines trigger probability based on the track angular distance from the Moon:

$$
\alpha=e^{\frac{(-\delta \theta)^{2}}{\sigma^{2}}}
$$

where $\delta \theta$ is the track declination from true Moon/Sun position and $\sigma$ is a suppression parameter that can be set in the configuration file (.fcl).

The smart prescale is based on the estimation of the expected rate for a given position of the Sun/Moon. The estimation is made with precision up to $1^{\circ}$ by creating a lookup table (stored in separate text file) with the expected rate given that it collects within $5^{\circ}$ from true Moon/Sun position with given angular suppression. The lookup table is visualized on Fig. 2.8. It is produced separately from the trigger and loads once in the trigger constructor. This mechanism was chosen since making this table is a computationally heavy process and takes around 10 minutes, so making it every time the trigger starts seems undesirable. Note that with the given method of making the lookup table, parameters such as track cut length and angular suppression become connected with each other and with the particular lookup table made with these 
parameters. The size of the lookup table file is $\approx 250 \mathrm{kB}$, which makes it easy to load.

A particular track is triggered if all of the following conditions apply:

- Track direction coincides with Moon/Sun direction within $5^{\circ}$ circle

- Probabilistic parameter calculated based on the ratio of expected rate from lookup table and desirable rate is true

- Probabilistic parameter calculated based on the angular suppression function is true

The main reason that the lookup table is computationally intensive is that collecting the rate in neighboring bins $\left(1^{\circ} \times 1^{\circ}\right.$ bins) involves a variable number of surrounding bins for different positions. Areas closer to $0^{\circ}$ zenith have more bins inside $5^{\circ}$ circle (see Fig. 2.9 for explanation). Thus, collecting the rate is more CPU demanding and should be calculated in advance rather than in the trigger system at start time.

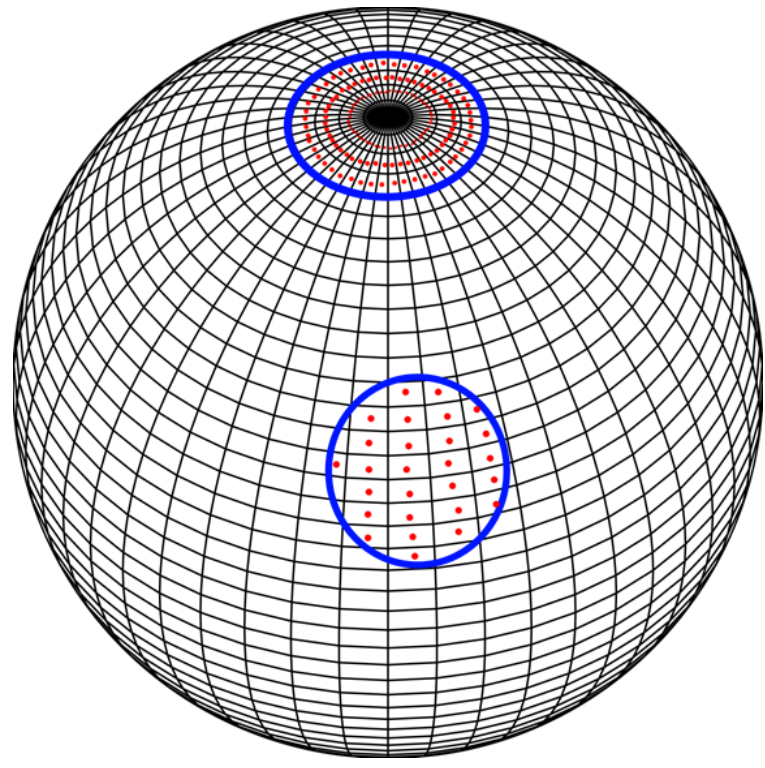

Figure 2.9: Illustration of collecting rate within a $5^{\circ}$ circle: different positions on the sphere can give different number of bins within the cone.

Since $1^{\circ} \times 1^{\circ}$ bins are used in the lookup table, the characteristic error with which we can have is $\approx 1^{\circ}$. Maximal relative error which we can get if true Sun/Moon position is close to the border of the bin and it's rate is different from one in bin center does not exceed $\approx 20 \%$ in most regions of the sky.

Overall, the smart prescale mechanism uses the following parameters set in the configuration file (.fcl):

- LengthCut: cut on length of a track $(*)$ 
- HitCut: cut on number of hits on a track $\left(^{*}\right)$

- AngularSuppression: parameter $\sigma$ in angular suppression function $(*)$

- AngularCut: maximal declination from true Moon/Sun position for track to be considered for triggering $(*)$

- AvgRate: desirable rate after smart prescale

(here items with an asterisk are bound together due to use of the lookup table based on these parameters)

The trigger is running with an active "smart prescale" algorithm, which reduces the trigger rate to $20 \mathrm{~Hz}$ total for the Sun and Moon trigger. Rates and other trigger parameters can be seen in the NOvA online monitoring system Ganglia- sample plots taken from this system are shown in Appendix C.

The latest version of Moon Trigger is accessible from the NOvA repository [12].

\subsection{Celestial Locator}

A main component of the Moon/Sun trigger is a code that allows one to locate celestial objects on the sky. We decided to make it a separate component so it could be used for other modules, such as the analysis module for upward-going muon trigger. In NOvA the way to create reusable components of this sort (which are not tightly connected to some analysis or trigger modules) is to make it an Art Service - in this case it's called the CelestialLocator service. More detailed explanation of how to use this module can be found in Appendix $\mathrm{D}$.

The CelestialLocator service uses Naval Observatory Vector Astrometry Software (NOVAS) as a key component. This software can be found at [13, 14]. The main part of NOVAS is the ephemerides file - basically, this is binary file storing trajectories of celestial bodies. There are few variations of ephemerides files (see [15]), the one used in NOVAS is DE405.

NOVAS software is accessible both in C and Python (and FORTRAN too) and Python code is a wrapper over $\mathrm{C}$. There are examples of code usage when you download NOVAS from the site (at least I used examples in Python, but there should be examples for $\mathrm{C}$ too). Main functions of NOVAS can be found again in Appendix D.

In the current version of CelestialLocator it allows the user to get the elevation and azimuthal angles of the Moon and the Sun in given Unix time (represented by number of seconds passed since 1.1.1970 :00:00:00 GMT) at the geographical position of NOvA Far Detector $\left(L A T: 48.378574^{\circ}\left|L O N G:-92.831051^{\circ}\right| H E I G H T: 367.6 m\right)$. Currently CelestialLocator service is used for Moon shadow analysis studies and also in upward-going analysis studies, since there position of the Sun determines whether current data belongs to background or to signal region (see description of upwardgoing analysis later in text).

The latest version of CelestialLocator service is accessible from NOvA repository [16]. 


\subsection{Moon shadow}

The cosmic deficit due to shadowing by the Moon based on collected cosmic pulser data was made by our group including me, L. Aliaga (Fermilab postdoc) and C. Group. The resulting plot of the cosmic rate distribution versus angular proximity to the Moon is shown on Fig. 2.10.

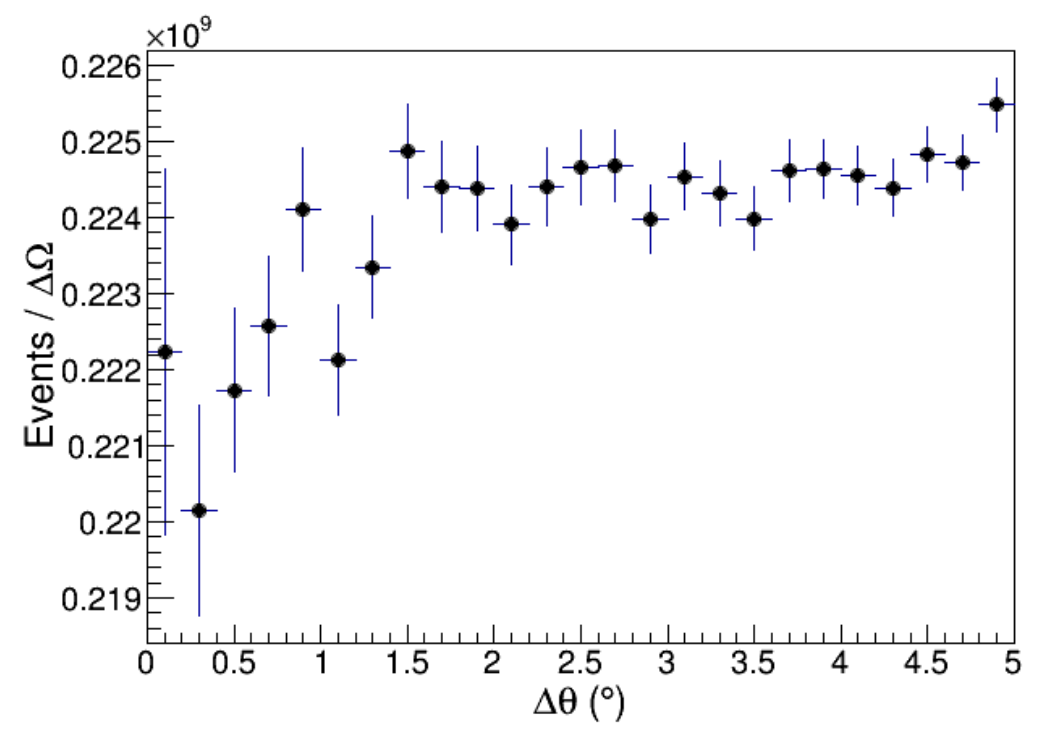

Figure 2.10: Plot of cosmic rate distribution versus angular proximity to the Moon direction. Plot made by L. Aliaga with cosmic pulser data.

The latest result on a Moon shadow from the Moon trigger is shown on Fig. 2.11 It was provided using about 1.5 months of Moon trigger data starting August 2016. This plot is preliminary and is under discussion. The more complete analysis with Moon trigger data is yet to come.

\section{7 $\quad$ Current state and plans}

The history of Moon trigger development is presented at Appendix E. Currently, the Moon trigger is running on the NOvA Far Detector and collecting data. Since data collection rate is about 4 times higher than that of the cosmic pulser, the trigger should be able to collect an amount of data necessary to have the same precision of the Moon shadow as we have now (after about 1 year of collecting the pulser data) in $3-4$ months.

Changes like introducing a more efficient prescale algorithm or changing the expected trigger rate, as well as changes regarding DDT tracking algorithm can be introduced throughout the trigger lifetime to make it more efficient and improve purity. 


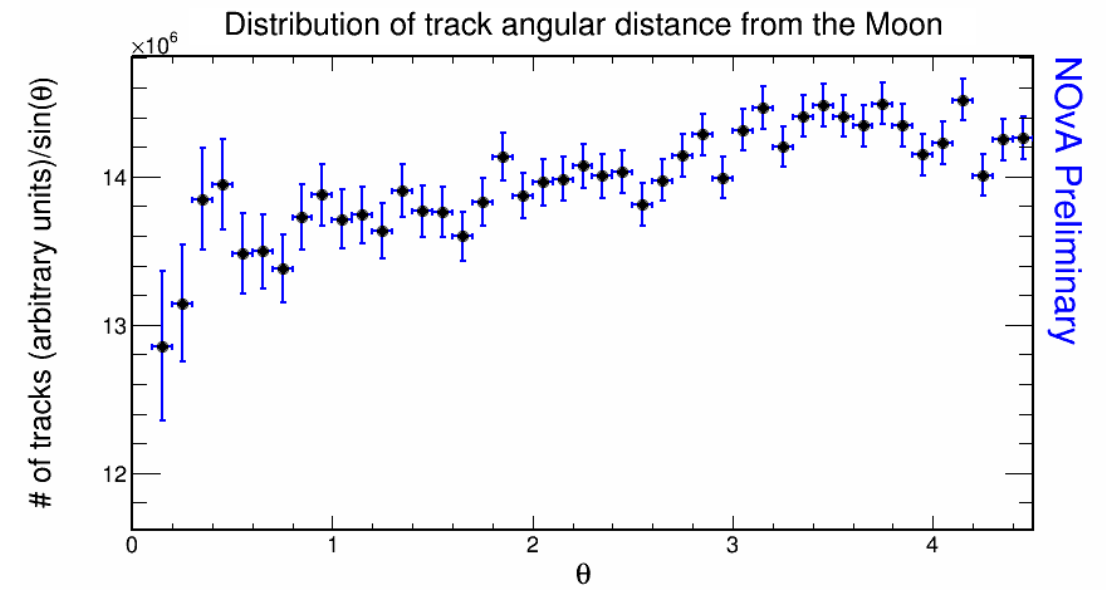

Figure 2.11: Plot of cosmic rate distribution versus angular proximity to the Moon direction. Plot made with the Moon shadow trigger data. 


\section{CHAPTER 3 UPWARD-GOING MUON STUDIES}

\subsection{Motivation}

Studies using upward-going muons is a part of the exotic program at the NOvA experiment. The goal is to search for signals of Dark Matter originating from the core of the Sun. One of the leading theories is that DM particles are WIMPs weakly interacting massive particles. The most promising candidate for a WIMPtype Dark Matter particle is the neutralino - a combination of fermionic fields of a supersymmetric extension of SM (gauginos and higgsinos). These hypothetical particles may be able to annihilate into cascades of SM particles. Since these particles are supposed to be rare, relatively stable (rarely decaying) and non-detectable, in order to make evidence of their existence, we need to find a good source of their annihilation products.

Note that the theory of WIMPs implies that these particles have mass and so it gives us hope that they can be gravitationally bound inside massive objects, and thus we may observe annihilation products coming from this place. One possible source is the core of the most massive object we can observe at an astronomically close distance - the Sun [7].

The main challenge of looking into properties of particles coming from the direction of the Sun is a huge background of cosmic particles, which form the main source of all tracks in the NOvA Far Detector with rates of about $100 \mathrm{kHz}$ (see the previous Chapter for more details). Thus, the most natural way to decrease levels of background is to look for particles going upward while the Sun is below horizon - see Fig. 3.1. In this case the Earth serves as a shield for all cosmic particles except for atmospheric neutrinos, which were formed on another side of the Earth from the Far Detector.

The UVA group has created the upward-going muon trigger. The main contributors to the creation of the trigger were Y. Oksuzian (UVA postdoc), R. Mina (UVA undergraduate student, now graduate student at Stanford) and C. Group (UVA professor). With the help of the trigger we hope to register upward-going neutrinos and to observe an increase of the flux from the direction of the Sun. This may be possible if DM particles are collected in its core and if they annihilate emitting high-energy neutrinos.

As mentioned above, the main source of background for DM studies using the upward-going-muon trigger is atmospheric neutrinos coming from the opposite side of the planet. So, the upward-going trigger may also be used to study atmospheric 


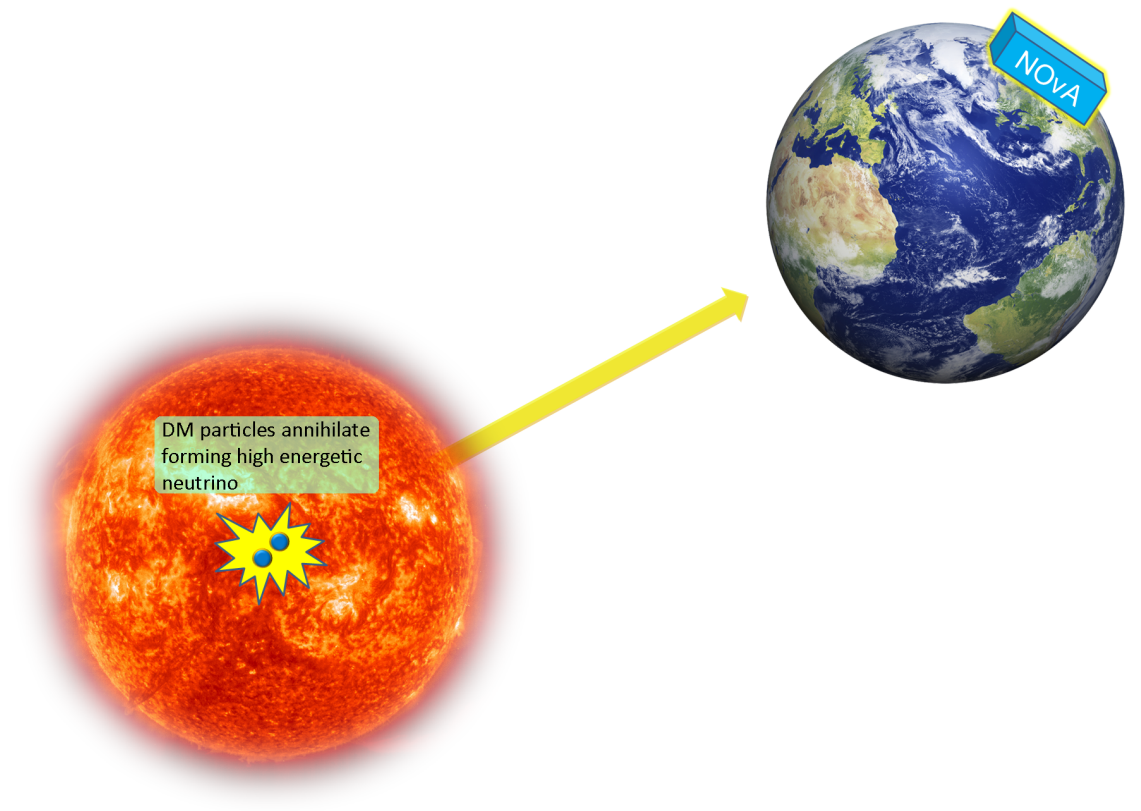

Figure 3.1: Dark Matter (DM) particles may annihilate in the core of the Sun producing a source of high-energy neutrinos

neutrinos. The rate of detectable upward-going atmospheric neutrinos is expected to be about $0.6 /$ day, according to a rough estimate and comparison to other experiments - you can find details in Appendix F.

The neutrino flux from dark matter annihilation is model dependent; however, energies from $0.5 \mathrm{GeV}$ to many $\mathrm{TeV}$ should be detected with high acceptance. For the high-mass signal hypothesis, NOvA will not be able to compete with the high acceptance of the IceCube detector [17]. For lower-mass scenarios (below $20 \mathrm{GeV}$ ) the Super-Kamiokande experiment currently has the best sensitivity [18, 19]. If an efficient upward-going muon trigger and sufficient cosmic ray background rejection can be achieved, NOvA will be competitive with SuperKamiokande for WIMP mass hypotheses below $20 \mathrm{GeV}$.

\subsection{Upward-going muon trigger}

The trigger allocation is given by physical possibilities of the NOvA DAQ system and is $10 \mathrm{~Hz}$, while the cosmic rate we get is around $100 \mathrm{kHz}$, hence we need to develop an algorithm allowing us to reduce the rate by 4 orders of magnitude while keeping good efficiency for registering upward-going muons.

To achieve this goal, we use timing information of all the hits in a track to estimate directionality and reduce the rate. A trigger of this type required the 2014 upgrade to the NOvA Far Detector readout from "singlepoint" to "multipoint" readout. This upgrade improved the single-hit timing resolution from about 125 ns to better than $15 \mathrm{~ns}$.

The upward-going muon trigger code is located in the NOvA DDT repository 
[23. It was first implemented and tested in August 2014, but did not run in a stable configuration until December 2014. Over the period of this sample, the through-going trigger fired at a consistent rate of approximately $1 \mathrm{~Hz}$ - see Fig. 3.2. Each triggered event is $50 \mu \mathrm{s}$ in length, so that the triggered sample contains about 1 part in 2000 of the total background activity during the exposure time. Activity in the NOvA far detector is dominated by muons from cosmic ray interactions above and around the detector [22].

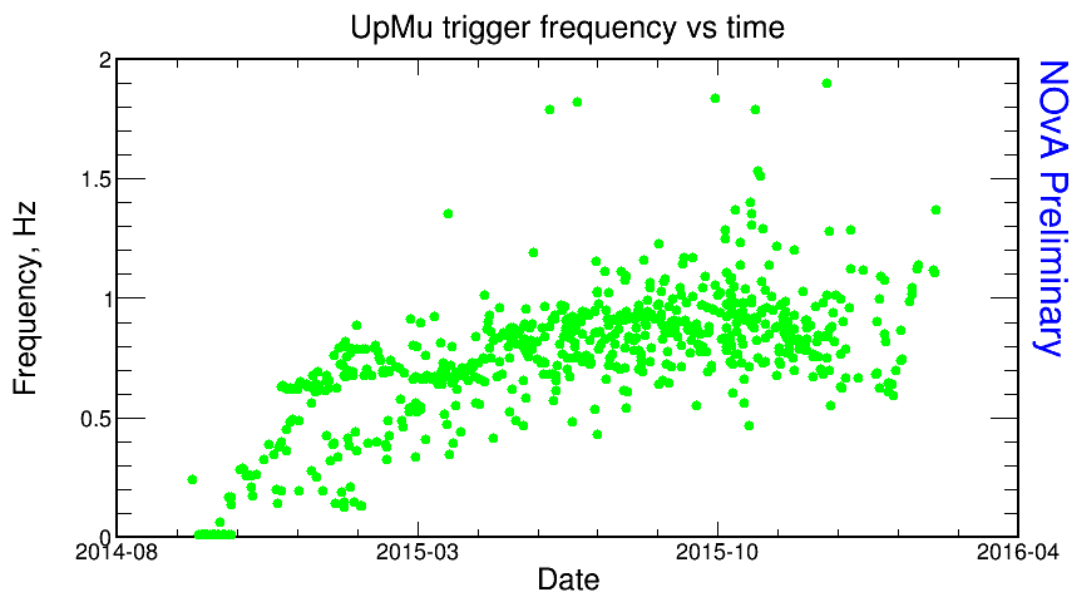

Figure 3.2: Upward going trigger rate through time, each point is averaged rate through 5 runs of NOvA Far Detector.

When estimating the efficiency of the upward-going muon trigger one should take into account the overall efficiency of the whole DDT system: some tracks can be misreconstructed and missed by the track reconstruction process in DDT system (it uses Hough tracker algorithm). Analysis showed that the DDT system efficiency was around $85 \%$ and it doesn't largely depend on track length and also is approximately constant throughout active time of the experiment. More complete description of DDT efficiency studies is provided in Appendix G.

\subsection{Timing resolution}

The electronic response to an incident particle depositing energy in a cell can be parameterized in a following form:

$$
f(t)=\alpha p e^{-\left(t-t_{0}\right) / T_{F}}\left(1-e^{-\left(t-t_{0}\right) / T_{R}}\right)
$$

where $\alpha$ is a proportionality factor, $T_{F}$ and $T_{R}$ are intrinsic falling and rising time of the response curve, respectively.

By performing a simple $\chi^{2}$ minimization, the value of $t_{0}$ can be extracted from multiple readouts on a single channel. For the purposes of the trigger, where hit processing time must be minimized, fit results were pre-calculated and tabulated such 
that the computationally expensive minimization need not be repeated for each individual hit. An example of fitting the electronics response curve to multiple readouts from a single cell hit is shown in Fig. 3.3 .

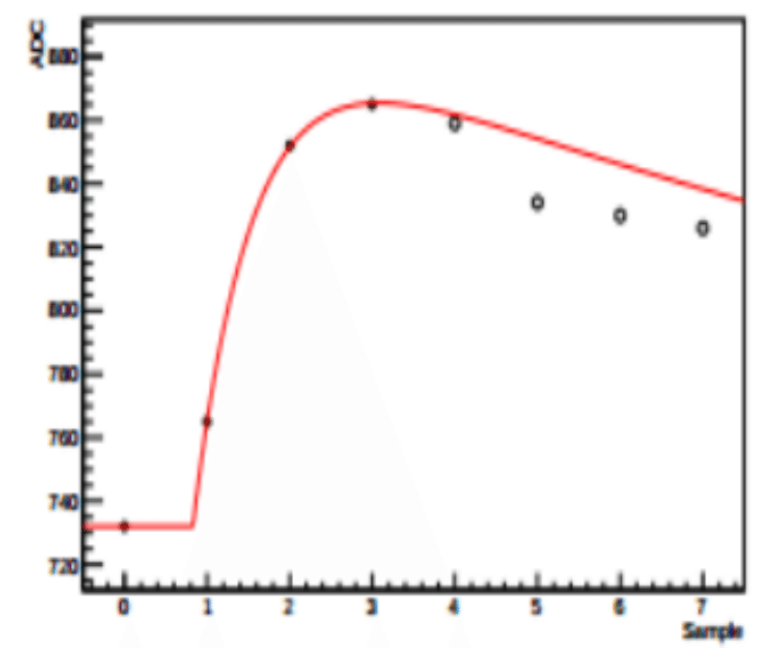

Figure 3.3: An example of fitting the electronics response curve to multiple readouts from a single cell hit.

Single-hit time resolution is measured by comparing hit times on a cosmic ray muon track. It depends on the hit ADC (general unit of signal amplitude after conversion into digital format) - this dependency is shown in Fig. 3.4. Using multipoint resolution technique, for high-energy hits the $\Delta t$ is measured to be better than $15 \mathrm{~ns}$ in the data using the four-point readout scheme. Overall you can see that switching from single-point time resolution to multi-point time resolution improves timing precision significantly.

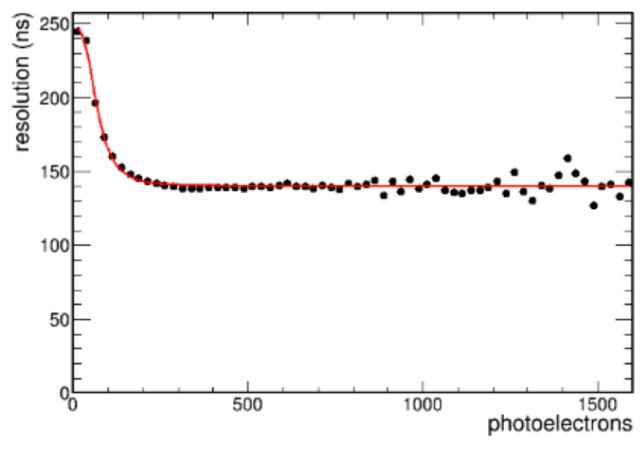

(a) single-point

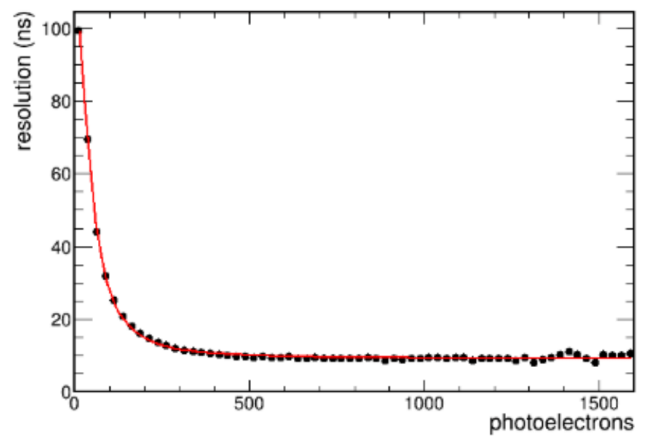

(b) multi-point

Figure 3.4: Single-hit timing resolution as observed in NOA far detector data with fourpoint readout, before (a) and after (b) multi-point fine timing implementation. 


\subsection{Track directionality}

The trigger is based on simple track ID, track quality cuts, and the timing information of the hits on a track. The tracks are reconstructed using the Hough transform algorithm, and are required to match in both $\mathrm{XZ}$ and $\mathrm{YZ}$ views. We start from the hit with lowest $y$ cell value, $y_{0}$, in the track in the $\mathrm{YZ}$ view. The measured time of the corresponding hit is defined as $T_{0}$. The observed and expected time of each hit on the track in the $\mathrm{YZ}$ view is therefore:

$$
T_{o b s}=T D C_{y_{i}} \cdot 15.625-T_{0} ; \quad T_{e x p}=T O F_{\mu} \frac{y_{i}-y_{0}}{y_{1}-y_{0}}
$$

Similarly, for the XZ view:

$$
T_{o b s}=T D C_{x_{i}} \cdot 15.625-T_{0} ; \quad T_{e x p}=T O F_{\mu} \frac{x_{i}-x_{0}}{x_{1}-x_{0}}
$$

where $x_{i}$ and $y_{i}$ are the cell numbers in $\mathrm{XZ}$ and $\mathrm{YZ}$ view, and $T D C_{x(y)_{i}}$ is the time measurement in TDC units, which is converted to $n s$ using the factor of $15.625 \mathrm{~ns} / \mathrm{TDC}$. $\mathrm{TOF}_{\mu}$ is the time-of-flight of the muon track defined as:

$$
T O F_{\mu}=L / 29.97
$$

where $L$ is track length in $\mathrm{cm}$, and $29.97 \mathrm{~cm} / \mathrm{ns}$ is the expected speed assuming that the muon is relativistic.

Since we require that each track is reconstructed and matched in both views, $\left(x_{0} ; y_{0}\right)$ and $\left(x_{1} ; y_{1}\right)$ must correspond to the lowest and highest points of the track respectively. In addition, we can estimate the missing coordinate for a particular hit in either view using the a 3-D matching requirement.

Since for each hit in each view we can estimate $(x ; y ; z)$ coordinates, we can calculate the distance from the hit to the APD readout end. The further the hit is located from the readout the longer it takes for the light to propagate and be detected by the APD. We are interested in the hit time of the muon passing through the extrusion, so we have to correct for the light propagation time in the fiber. The speed of light in the fiber is measured to be $15.3 \mathrm{~cm} / \mathrm{ns}$. To date, the offline timing calibrations are not available online, so we included our own code in the trigger for the calibration.

We can use Eqns. 3.2 and 3.3 to produce the distribution of the expected $v / s$ observed time for each track. An example of expected v/s observed time distribution is shown in Fig. 3.5. The distribution is produced using a reconstructed upward-going muon track simulated with WimpSim [20, 21]. As can be seen, the points follow a rising trend with a slope value consistent with the upward-going track hypothesis. It is clear from the figure that the fitted slope value can be used to estimate the muon direction (up or down). As shown in Fig. 3.6, the slope values for cosmics and WimpSim MC samples are consistent with the downward- and upward-going hypothesis, respectively. In the relativistic limit, it is safe to assume that there are only two options for the slope values. Therefore, we can fit the time distribution on 


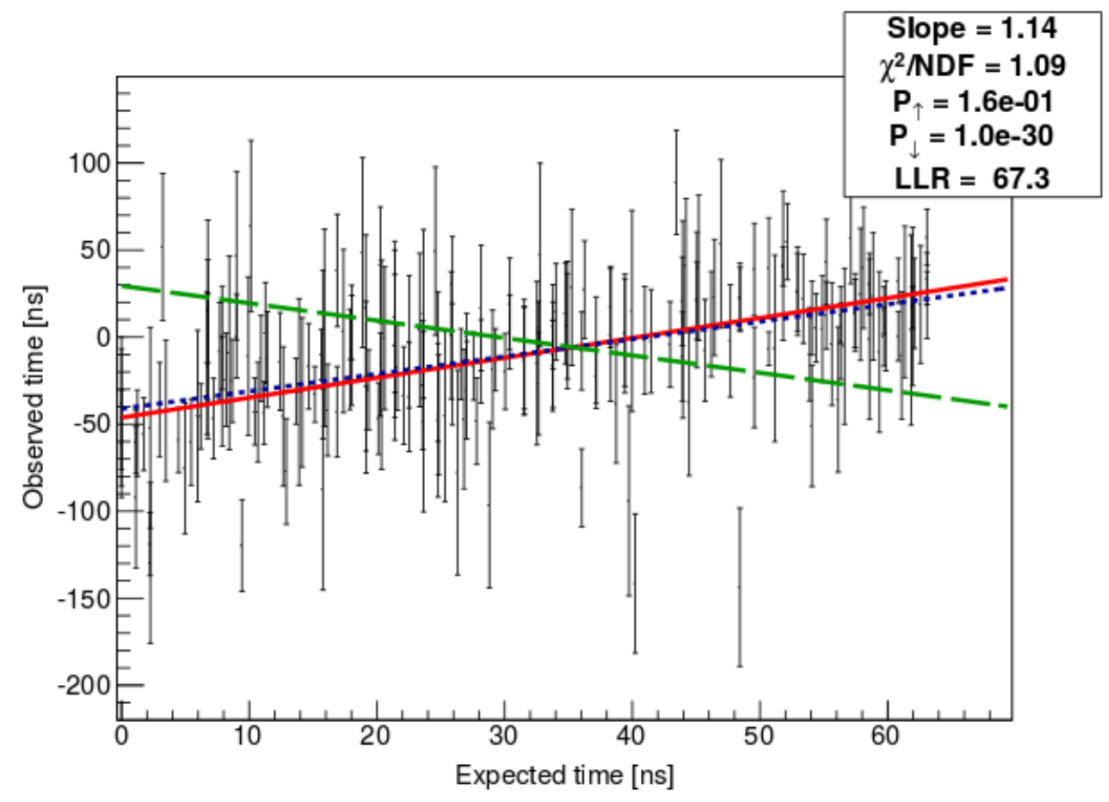

Figure 3.5: The expected versus observed time distribution for an upward-going muon track reconstructed in the NOvA far detector, using fine timing. The linear unconstrained fit (red solid line) has slope value close to 1 . The fit with the upward-going track hypothesis (slope $=1$ ) is shown as the blue-dashed line. The fit with the downwardgoing hypothesis is shown in the green dashed line and has a very poor probability.

Fig. 3.5 with fixed values of slopes. For the upward-going track the fit with the slope constrained to "1" results in a good $\chi^{2}$ probability value of the fit, $P_{\uparrow}$. However the fit with slope of -1 yields a low probability value, $P_{\downarrow}$. Using the probability values from the fits with the fixed slope value, we can form a log-likelihood ratio (LLR):

$$
L L R=\log \left(\frac{P_{\uparrow}}{P_{\downarrow}}\right)
$$

The LLR distributions for the cosmic and WimpSim MC samples are shown in Fig. 3.7. From this distribution, it is clear that a cut on LLR slightly above zero will reduce the cosmic background by the desired amount while preserving a high signal acceptance. Note that the WimpSim sample used is for dark matter with a $20 \mathrm{GeV}$ mass annihilating through the $b \bar{b}$ channel. As such the neutrinos from the b-meson decay produce muons which, on average, have a much lower energy compared to the cosmic ray muons. So, the signal shown here has shorter tracks with less hits than the events in the cosmic ray muon sample. This explains why the LLR for the signal has a larger component close to zero than the cosmic sample.

The LLR yields better performance for cosmic background rejection for the same signal acceptance in the regime where the cosmic rejection is sufficient (at least four 
orders of magnitude), compared to a cut on the best-fit slope. For example, for a signal acceptance of 0.7 the background rejection is about a factor of three better for the LLR. At this point the MC predicts background rejection of close to five orders of magnitude. In addition to being a more powerful discriminator as observed in the MC studies, the LLR estimator is more robust to mis-reconstructed tracks which will be an important feature in real data. Since mis-reconstructions will result in time distributions that follow neither the upward- nor the downward-going hypothesis, the result of mis-reconstruction will yield LLR values close to 0 , and not values consistent with a high-probability for being upward-going.
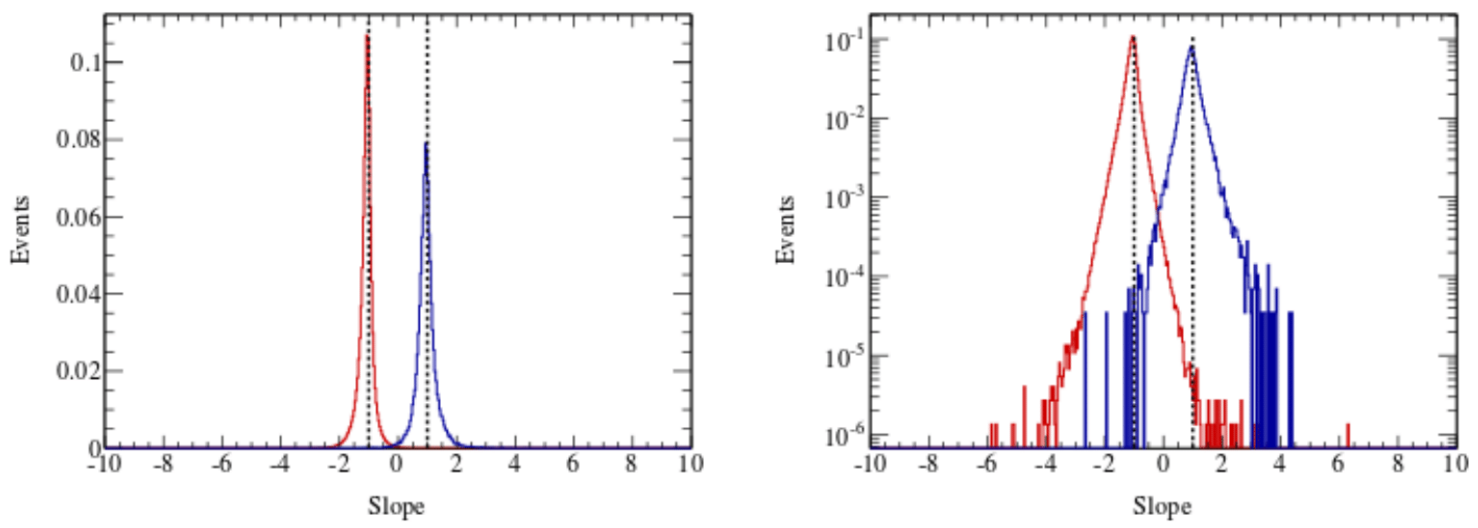

Figure 3.6: The slope distributions for cosmics (red) and WIMPSim (blue) MC samples.

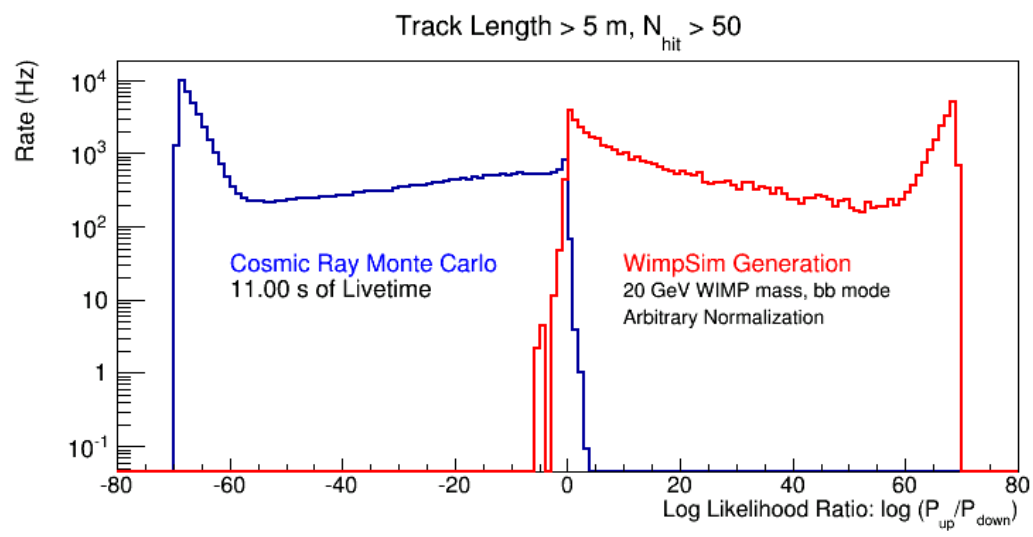

Figure 3.7: The LLR distributions for cosmics (red) and WIMPSim (blue) MC samples. Note that only tracks longer then $5 \mathrm{~m}$ and with more than 50 hits are included. 


\subsection{Analysis}

The analysis is performed using several offline analysis modules: UpMuAna, UpMuAnalysis, UpMuRecoAna (for their location in NOvA offline repository see [24]). In principle, analysis modules use the same logic as the trigger module, but act on reconstructed files (with window tracker used for track reconstruction).

The plan involves performing a blind analysis for the WIMP search. In order to accomplish this, the upward-going-muon analysis utilizes three basic analysis regions for splitting the acquired data.

Using metadata parameters we can split out data into several signal and control regions as illustrated in Fig. 3.8:

- Signal Region (Night): Sun is at least 10 degrees below the horizon.

- Primary Control Region (Day): Sun is at least 10 degrees above the horizon.

- Secondary Control Region (Twilight): Sun is within 10 degrees of the horizon.

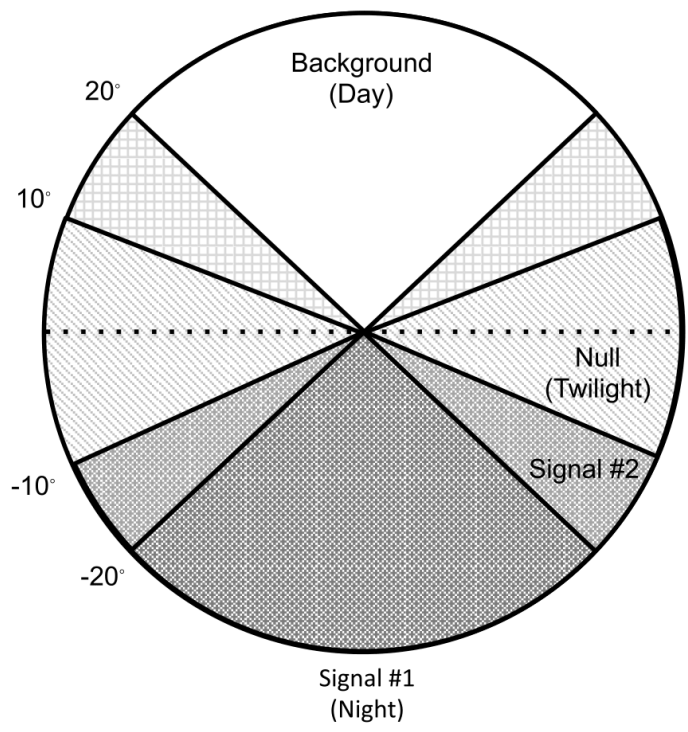

Figure 3.8: A diagram of the various signal and control regions used in the analysis.

Unblinding the analysis will include a stage of checking that detector observables are stable between the day and night samples.

To exclude some events which can spoil the analysis and provide incorrect decisions on choosing upward-going candidates, data quality checks were performed, which excluded about $1.7 \%$ of acquired data. See details for data quality checks in Appendix $\mathrm{H}$. Appendix I provides full set of parameters and cuts on those used in upward-going trigger and analysis modules. 


\subsection{ICHEP 2016}

The latest (to date) analysis of the upward-going muon trigger data covers a period of 424 days from December 2014 to late March 2016. The total livetime of this sample is 277.35 days.

Apart from the analysis itself, various data sanity checks were performed to assure that data is valid. Also, special attention was paid to the difference in signal and background data samples.

The difference between day (background) and night (signal) emerges from the difference of Sun elevation angle distributions. For example, in the fall, the Sun spends more time below the horizon, and its negative elevation angles have larger absolute values than positive ones. So, if you compare $\Delta \theta$ for day tracks pointing at the Sun above the horizon with $\Delta \theta$ for night tracks pointing to the Sun below the horizon you will have a different path in the sky of the Sun in the two samples. Fig. 3.9 shows a difference between azimuth and zenith angle distributions for the day and the night sample.
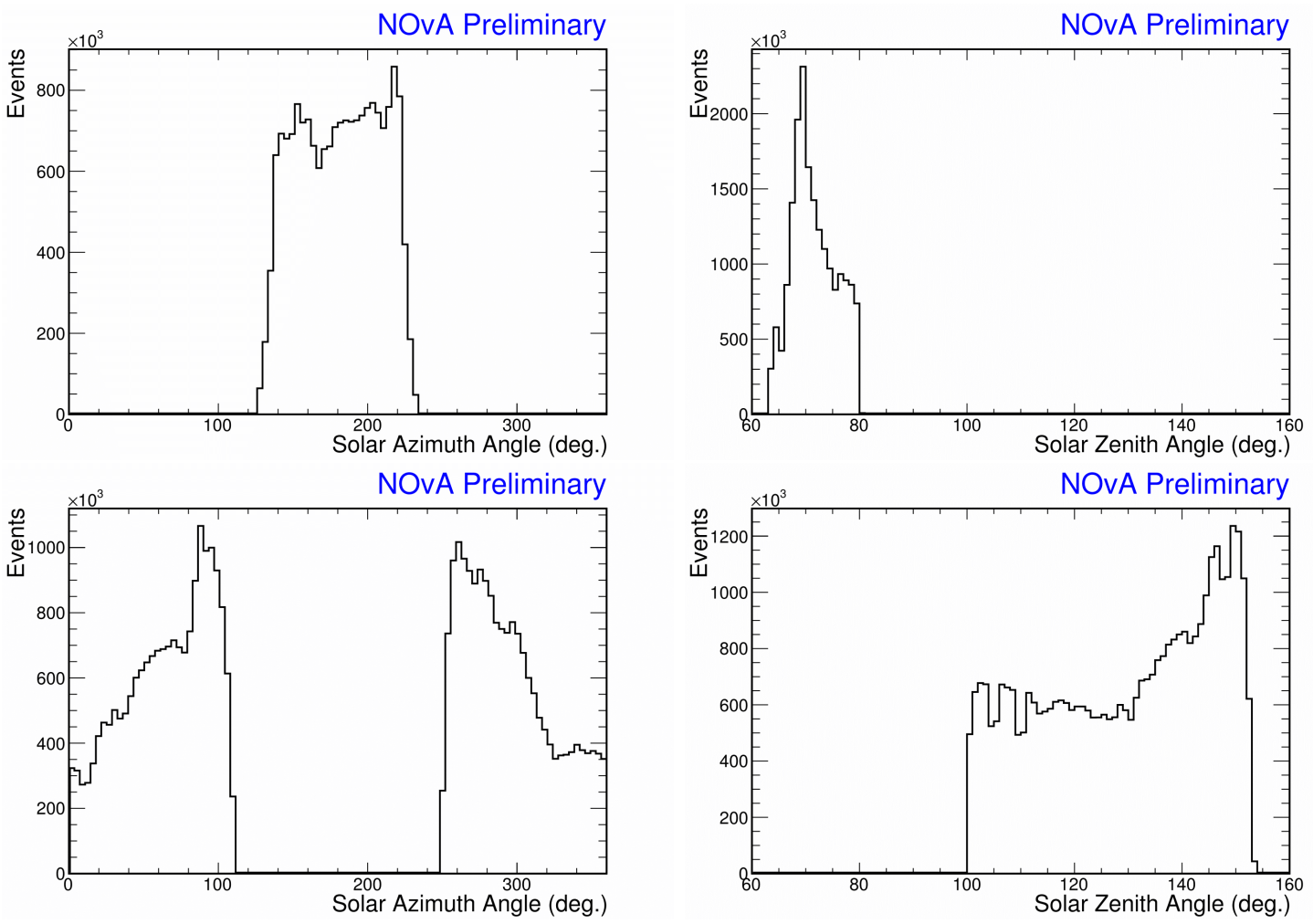

Figure 3.9: The azimuth and zenith angle of the Sun in the day (top) and in the night (bottom).

To address the issue of day/night sample difference, we implemented a 'phantom' position for the Sun in the data control region. The idea for the 'phantom' position it to draw the Sun position randomly for the day sample, based on the distribution that it follows at night. Using the 'phantom' position of the Sun in the day control 
region we compare the $\Delta \theta$ distribution for the day and night samples in the cosmic ray unbiased sample in Fig. 3.10. We observe a weak systematic trend that changes the ratio by about $2 \%$ over the range of the distribution. Regardless of the source, a two-percent bias will be a very small effect compared to our statistical limitations in the dark matter search. So, when we complete the search analysis we plan to include this as a systematic uncertainty.
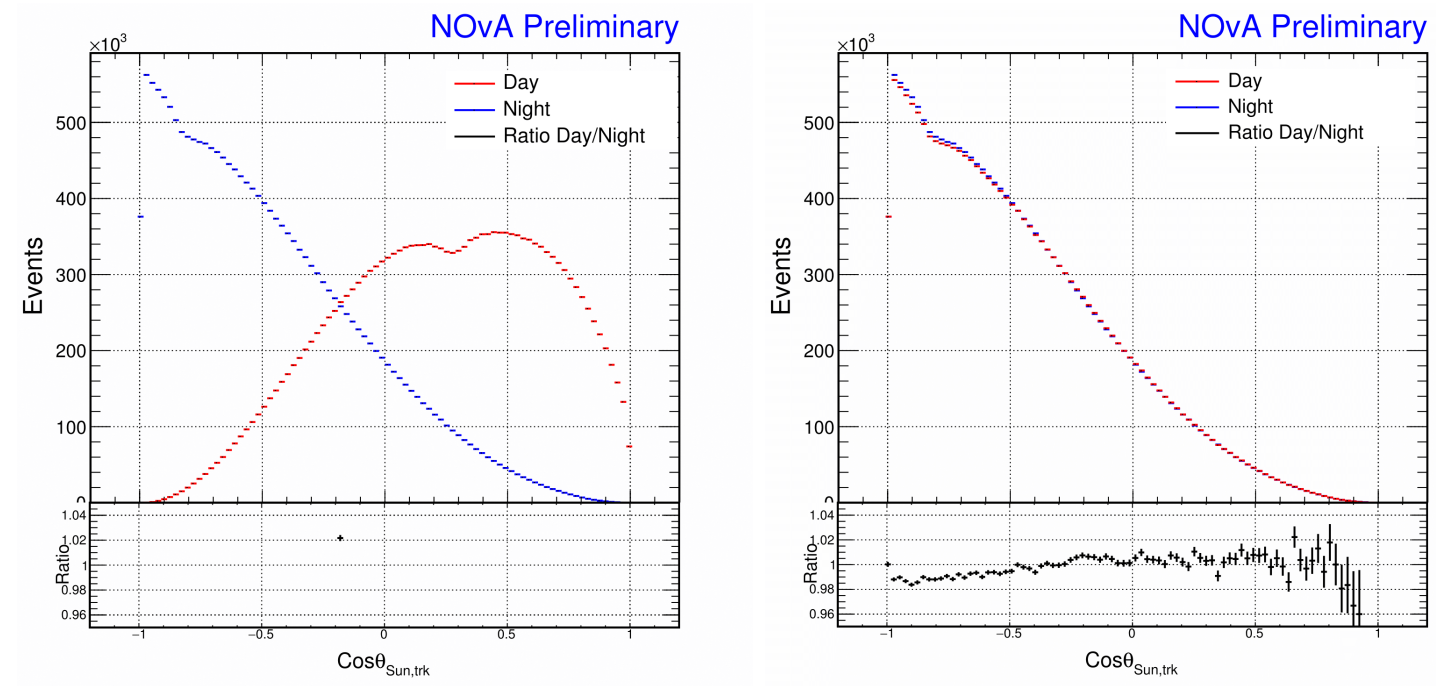

Figure 3.10: The $\cos (\Delta \theta)$ distribution for the day and the night samples. $\theta$ is the angular distance between the track and the solar position. Left plot shows initial distribution, right plot shows distribution with the Sun position being drawn randomly from the distribution that it follows at night.

With the result of Fig. 3.10, we can be confident that the shape obtained an in the day control region should match the shape obtained in the night sample to $2 \%$ or better in the absence of an upward-going signal correlated with the Suns position.

Using the data acquired by the upward-going trigger algorithm and providing analysis using cuts discussed in the previous section and Appendix I, we found some upward-going event candidates in the background sample. After applying all cuts, the size of the candidate subsample is 33 tracks (see also Table I.6 in Appendix I). Of those that remain, $40 \%$ are not conclusively upward-going due to a possible misreconstruction in which two unrelated but overlapping muon tracks create ambiguity in the reconstruction, as shown in Fig. 3.11. The example of a good upward-going muon candidate, which is also among these 33 tracks, is shown on Fig. 3.12 .

The elevation angle distribution for full data sample and subsample of 33 upwardgoing candidates is shown on Fig. 3.13 .

This result is a proof of concept that the idea of upward-going muon analysis really works. The result was presented at recent International Conference for High Energy Physics (ICHEP 2016) in form of a talk (A. Tsaris, postdoc at Fermilab) and a poster (C. Principato, UVA graduate student). 


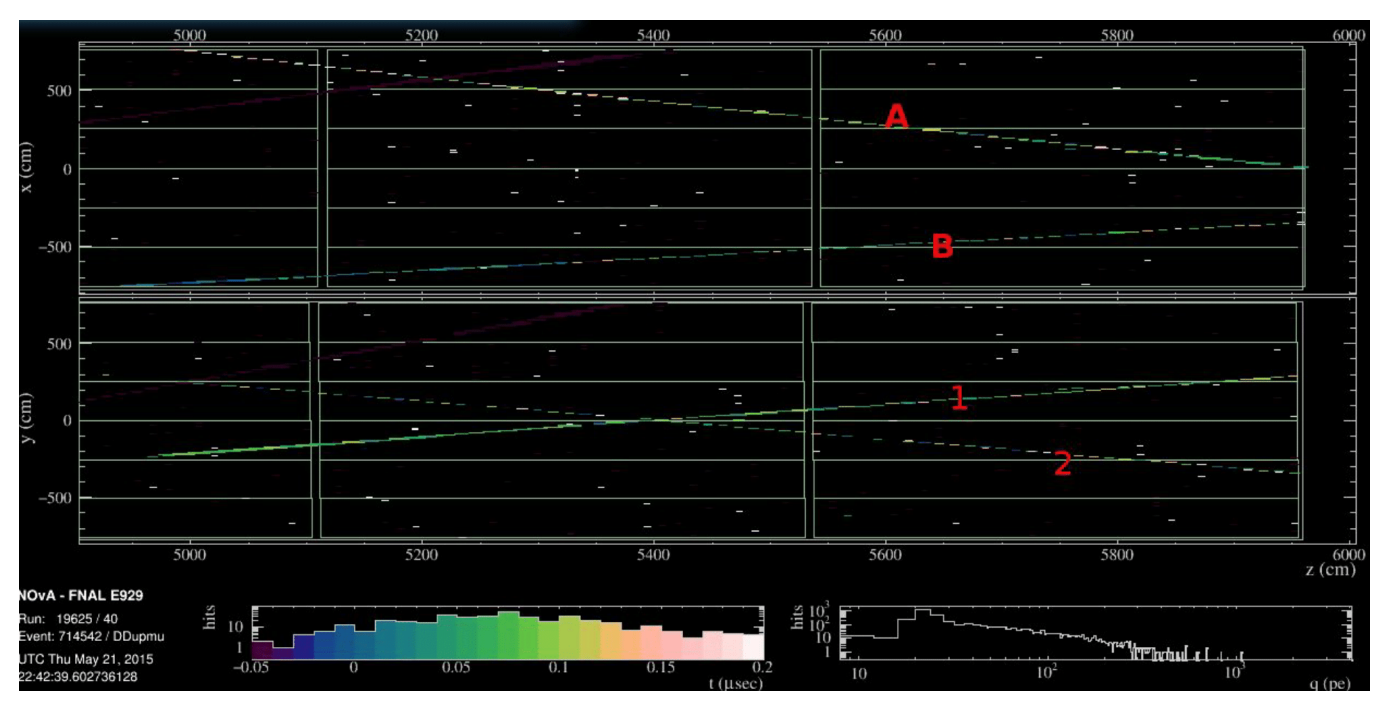

Figure 3.11: An example of an event that is prone to a possible misreconstruction. Two overlapping muon tracks with similar extent in the $z$-dimension and near coincidence in time (hits are colored by time). 2D track objects from each view must be merged to produce a full 3D object, but in this case it's ambiguous: is the correct matching $(\mathrm{A} 1, \mathrm{~B} 2)$ or $(\mathrm{A} 2, \mathrm{~B} 1)$ ?

The analysis presented at ICHEP 2016 demonstrates that NOvA is capable of isolating a sample that is likely rich in neutrino-induced upward-going muons. Furthermore, we have demonstrated NOvAs ability to point to celestial objects. We plan to use a daytime control region to measure the background for a WIMP dark matter search using upward-going muon data collected when the Sun is below the horizon.

Further accumulating of data opens the door to a competitive dark matter search and potentially sets the stage for a program to study atmospheric neutrinos by the NOvA experiment.

\subsection{Current state and plans}

Currently the upward-going trigger is working and collecting data from the NOvA Far Detector. The nearest plan for upward-going muon analysis is to provide thorough analysis of a signal region, as well as make some advances to the current analysis algorithm, such as getting rid of misreconstructed tracks which form the main reducible background in the final sample. The more distant plan is to collect more data for further analysis and make more definite statements about Dark Matter. 


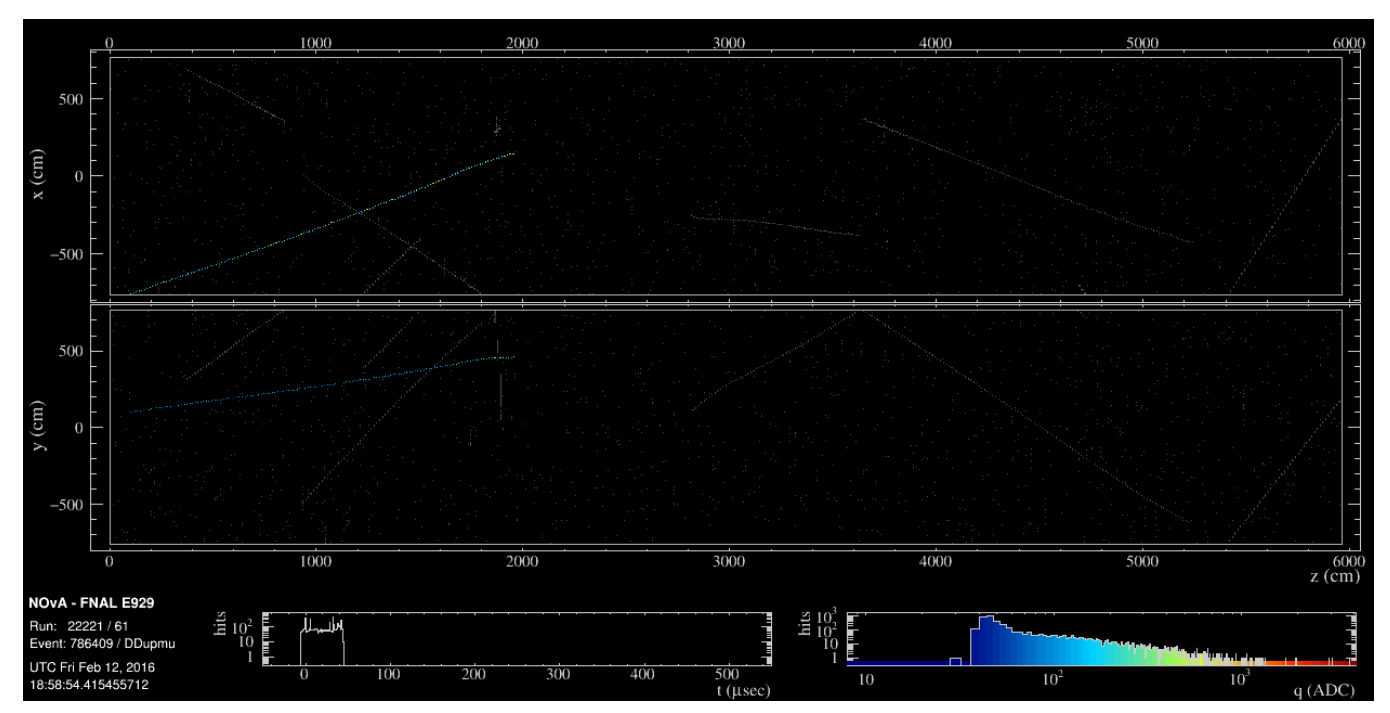

Figure 3.12: An example of an event that is a good candidate for upward-going muon. The track (highlighted) passed all cuts and showed good probability to be going up.
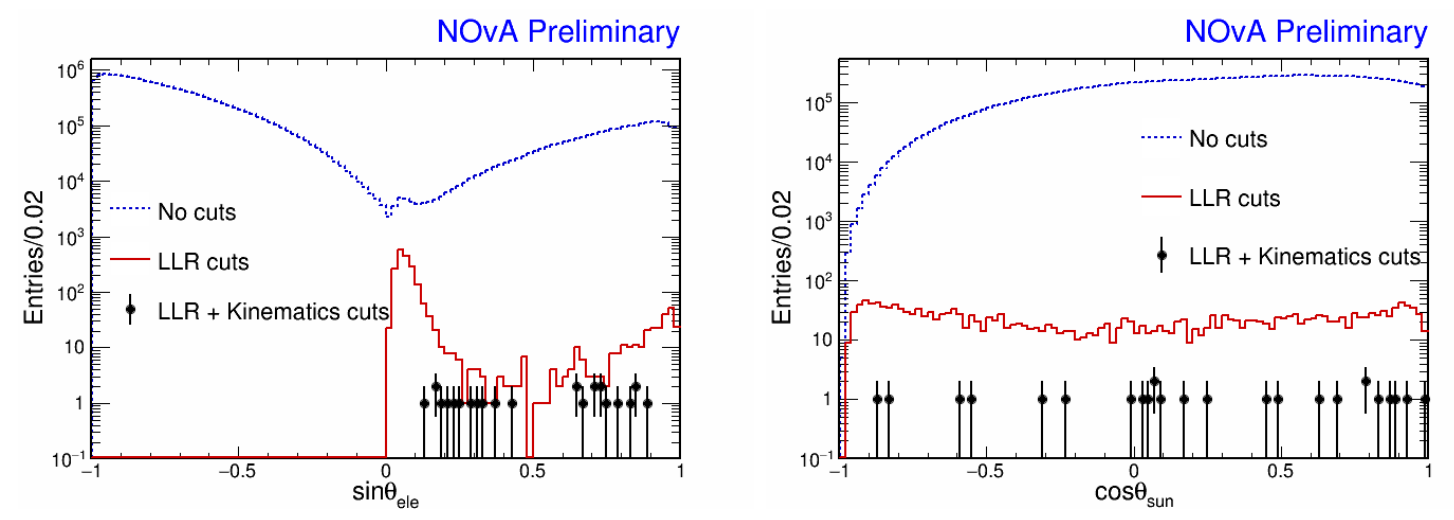

Figure 3.13: Distribution of the variables with respect to the elevation angle of the track, where 0 is horizontal, -1 is downward going, 1 upward going 


\section{Chapter 4 DDT EVENT DISPLAY}

\subsection{Motivation}

An event display is a tool for displaying hits in NOvA detectors. The main EventDisplay module is written in $\mathrm{C}++$, relies on the ROOT framework and is a part of offline software release. An example of the visualization is shown on Fig. 4.1. The code of this module can be found in [29]. Even though the event display is one of the most useful tools used in NOvA collaboration, it has some limitations.

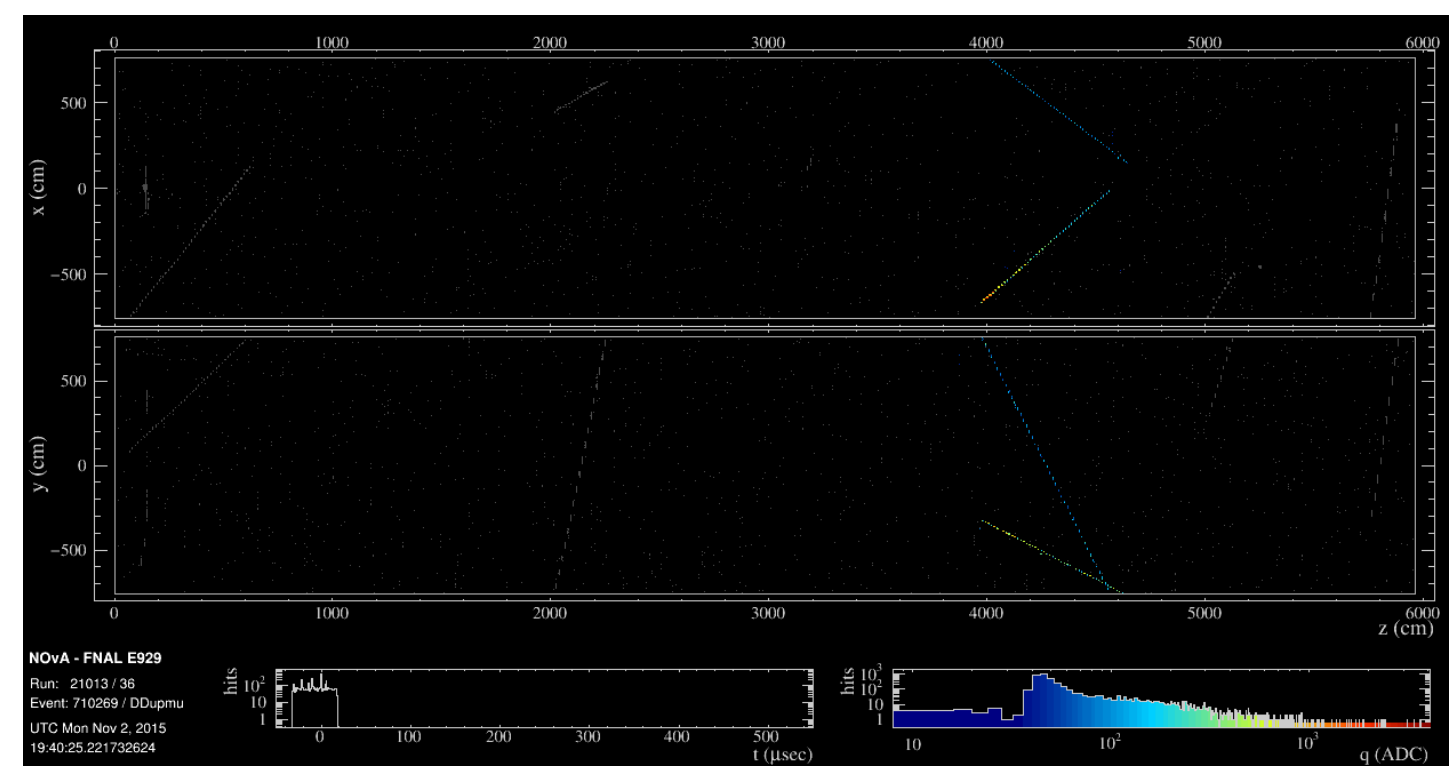

Figure 4.1: Example of visualization provided by EventDisplay module.

First, it was designed to display hits, so it works with very basic data format which stores hits in ROOT format - so called "artdaq" files. These files don't contain information on reconstructed tracks, but only information on individual hits and slices (chunks of hits separated in time and space). Navigation is possible through slices and time for particular event recorded in artdaq file. Navigation through tracks is impossible since the conventional event display doesn't know anything about them.

Second, EventDisplay can only be used for offline system files and cannot work with file format of the DDT system - so displaying tracks which were reconstructed by the DDT system is impossible with this tool. 
Finally, for some particular tasks, such as the Moon shadow analysis, it can be useful to visualize 3D objects to see their location and relative positions. The conventional event display only supports $2 \mathrm{D}$ visualization of detectors, presenting them in 2 views ( $\mathrm{XZ}$ and $\mathrm{YZ}$ views).

Changing the code of the EventDisplay module is a complicated task, especially if one wants to address the mentioned problems - it requires major changes to the core code of the module. To address these difficulties, I decided to create a separate tool, which is not as universal and powerful as the original EventDisplay, but is providing these special possibilities. Hence a 3D event display (aka DDT Event Display) and supporting DDT and offline modules [30]-31] were designed. They make it possible to display DDT tracks (also, reconstructed tracks) in both 2D and 3D formats. You can see the example of track visualization provided by DDT Event Display on Fig. 4.2 .

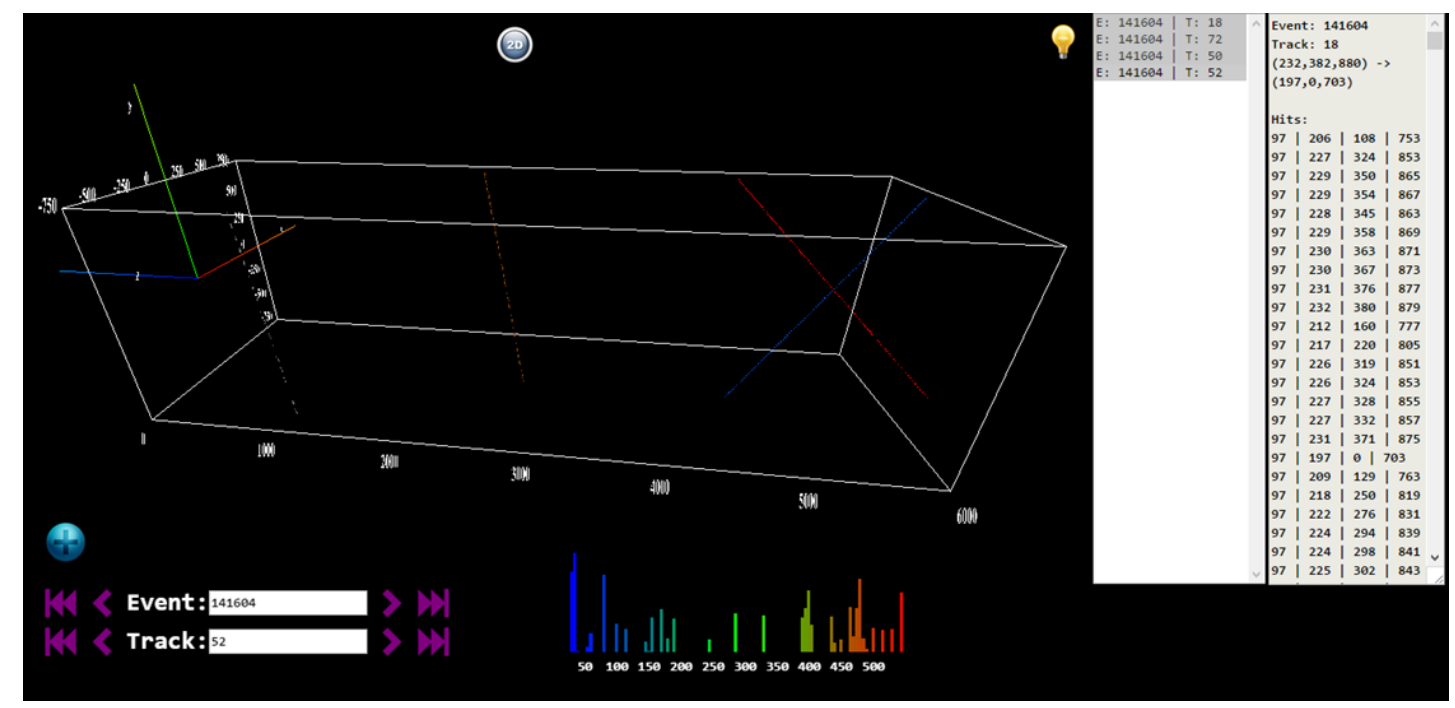

Figure 4.2: Example of visualization provided by DDT Event Display.

The DDT Event Display is designed to run from browser and accepts text data files produced by offline and DDT modules. This new event display doesn't use ROOT, so it doesn't need to be rebuilt with new ROOT versions. To see the main parts of the event display and its usage - see Appendix J.

\subsection{Choice of technologies}

To design the new event display I decided to discontinue a line of creation of software using ROOT-related tools, since in my opinion, ROOT is not the best choice for providing complex visualization, since the code turns out to be very specific and hard to change. Also, in this case it introduces a ROOT dependency and needs to be rebuilt when the underlying version of ROOT changes.

At the same time, technology used for this task should be common enough so everyone from the collaboration can use it regardless the operational system they use 
(usually, different Linux distributions and versions of MacOS). The natural choice for the technology with these requirements was visualization in a browser using JavaScript libraries. It automatically makes the solution cross-platform, it also removes dependencies on compilers and frameworks. All necessary calculations can be made locally in the browser.

The architecture of the solution can be logically split into 3 parts:

- "Front-end": JavaScript code and HTML/CSS markup

- Data in text format

- "Back-end": modules in offline/DDT systems designed to produce data in the right text format taking ROOT data files as input

The usage then is to run modules through the detector data of interest, produce the output text file and then drag and drop it to the browser window in the DDT Event Display page. By default the DDT Event Display will work in any browser which supports WebGL (it can require turning on some configuration settings in Safari and Firefox, but in tests it worked out of the box in Opera and Google Chrome).

For front-end part, the following JS/CSS libraries were used:

- THREE JS: 3D visualization

- D3JS: making histograms

- jQuery: hiding/showing elements to switch between views + easy access to html elements

- Bootstrap: CSS library for better markup

Since the DDT Event Display was created as a tool for a particular set of tasks and also due to the use of common technologies with extensive documentation and volume of examples, its code can be (comparatively easily) modified to add new features.

\subsection{DDT and offline modules}

Offline DDTEventDisplay and DDT xxx module are standard ART analyzer modules. They take reconstructed/artdaq/DDT file and produce a text file DDTEvd_Data.txt in current directory.

The produced data format reads:

E 3351261448784733

$\mathrm{T}(4,383,475)(3,321,496)$

$79 / 4 / 383 / 475 / 1,80 / 4 / 377 / 477 / 1,80 / 4 / 365 / 481 / 1,80 / 4 / 371 / 479 / 1, \ldots$

$(4,369,475)(2,323,496)$ 


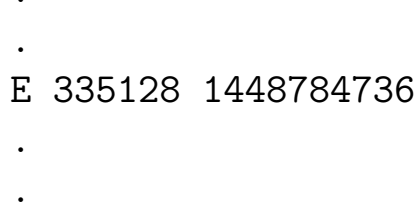

The scheme of data is as follows:

- $\mathrm{E}$ is the indicator of event header. Event header contains 2 numbers: event number and time offset from NOvA epoch in seconds (NOvA epoch is 01.01.2010 00:00:00 GMT).

- $\mathrm{T}$ is the indicator of track header. Track header contains 2 set of numbers in brackets: coordinates of the start and the end points of the track (in cell and plane numbers).

- Line after track header contains coordinates of individual hits (again in cell and plane numbers) separated by comma. Each coordinate contains 5 numbers separated by '/' sign, these numbers are (listed in same order): time offset from beginning of the event in $m s, x$ coordinate, $y$ coordinate, $z$ coordinate, index of view ( 1 for $\mathrm{XZ}$ view and 2 for $\mathrm{YZ}$ view).

Note that objects of the Track3D class used to produce coordinates of individual hits. These objects have 3D start and end points, but contain hits in form of DAQhits, which are 2D objects having information about hit's plane/cell/view - so only 2 coordinates are known. Each DAQhit is then transformed in 3D object in assumption that track is a straight line.

Size of resulting text file for cosmic pulser file is approximately $350 \mathrm{Mb}$. Front-end part of the DDT Event Display has been proven working with $150 \mathrm{Mb}$ files, so its better to split or truncate output files, which is easily doable with text files.

\subsection{Use cases}

The DDT Event Display was used to compare tracks produced by different trackers for DDT system efficiency study as part of upward-going muons analysis - see Appendix G.

Also, the advantages of the 3D visualization was used for Moon shadow studies to check that the directionality of tracks and prove they point to the Moon position - see Fig. 4.3 (in this case I made a modification in the front-end part of DDT Event Display to put the Moon above the detector in the right position).

Ability of DDT Event Display to overlay and compare tracks was also useful to check bug fix in the Moon shadow trigger - see Fig. 4.4. 


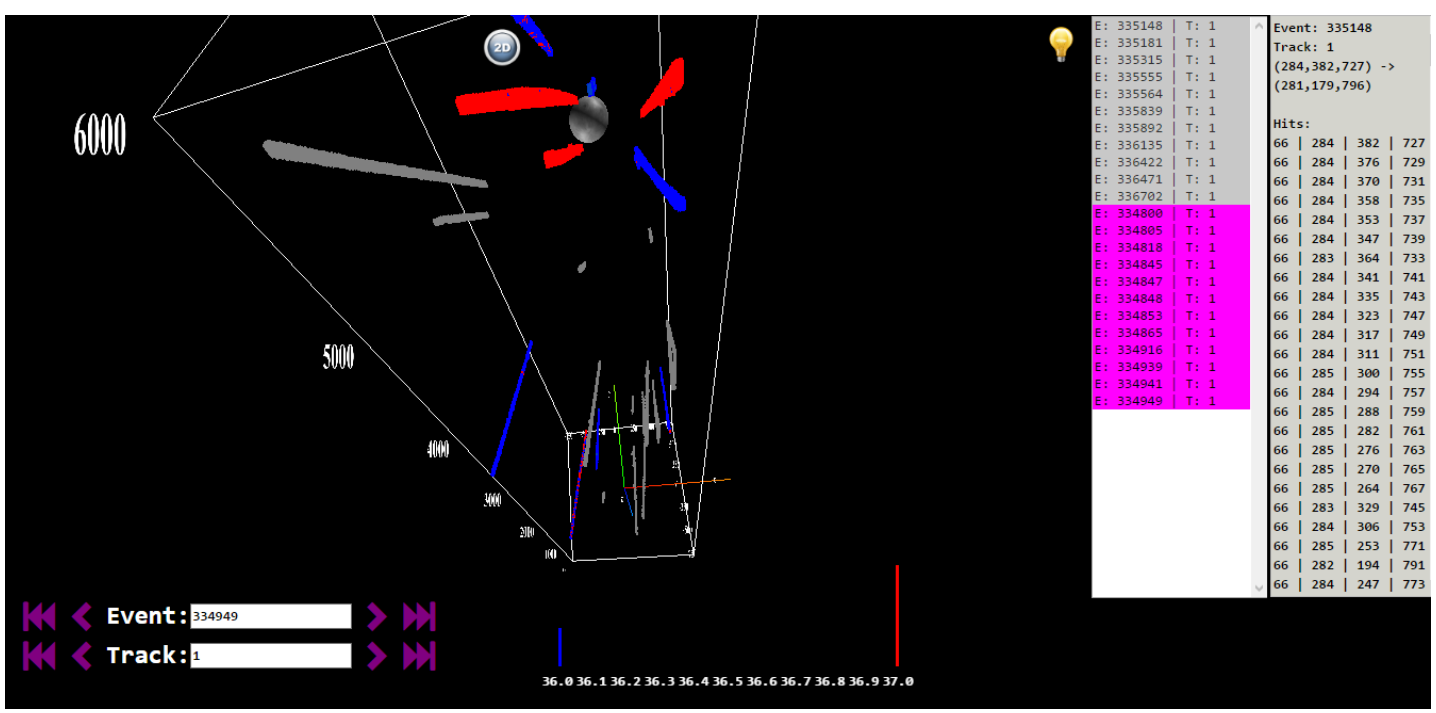

Figure 4.3: DDT Event Display visualization of tracks pointing to the Moon. Visualized data is recorded by the Moon trigger.

\subsection{Current state and plans}

Currently DDT Event Display is fully functional and can be used by everyone in the collaboration. Some improvements were on my mind at the moment of the release:

- Better markup

- Loading big files. Improving this is not that easy since it requires loading data from a file as a stream rather than loading all data at once.

- Zoom in $2 \mathrm{D}$ view

- Switch between cm and cell \#

These changes are useful, but since DDT Event Display is a tool with limited applicability and will probably be used by the DDT group only they are not an extremely high priority. 

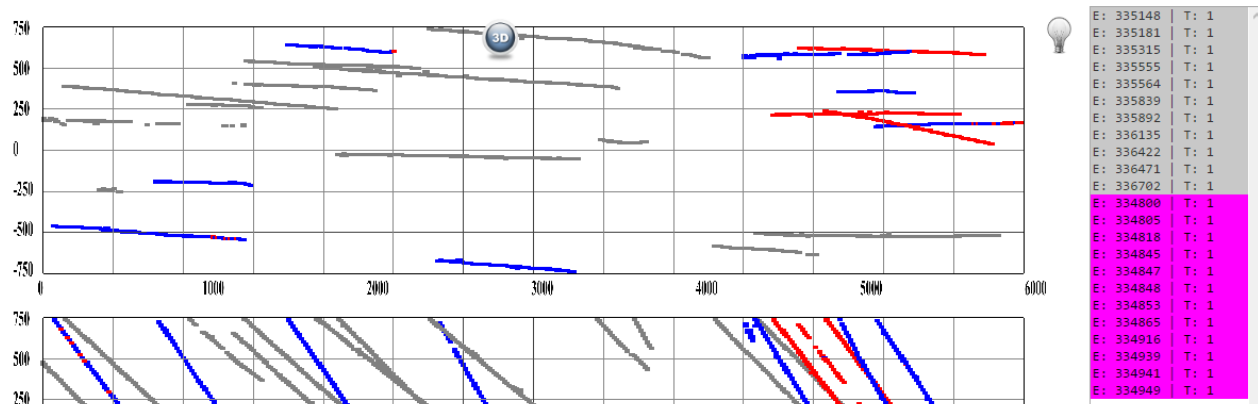

Event: 335148

Track: 1
$(284,382,727)$->

$(281,179,796)$

Hits:
66 | $284|382| 727$ \begin{tabular}{l|l|l|l|l|}
666 & 284 & 376 & 729 \\
66 & 284 & 370 & 731
\end{tabular} \begin{tabular}{l|l|l|l|l|l|l|l|l|l|l|l|l|}
666 & 284 & 370 & 731 \\
66 & 284 & 358 & 735
\end{tabular} \begin{tabular}{l|l|l|l|l|l|l|l|l|l|l|l|l|}
66 & 284 & 358 & 735 \\
66 & 284 & 353 & 737
\end{tabular} \begin{tabular}{l|l|l|l|l|l|l|l|l|}
666 & 284 & 347 & 739
\end{tabular}

\begin{tabular}{l|l|l|l|}
66 & 283 & 364 & 733
\end{tabular}

\begin{tabular}{l|l|l|l|l|}
66 & 284 & 341 & 741
\end{tabular}

\begin{tabular}{l|l|l|l|l|l|l|}
666 & 284 & 335 & 743
\end{tabular} \begin{tabular}{l|l|l|l|l}
666 & 284 & 323 & 747 \\
\hline 66 & 284 & 317 & 749
\end{tabular} \begin{tabular}{l|l|l|l|l|l|l|l|l|l|l|l|}
666 & 284 & 317 & 749 \\
66 & 284 & 311 & 751
\end{tabular}

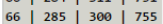
\begin{tabular}{l|l|l|l|l|l|}
66 & 284 & 294 & 757
\end{tabular} \begin{tabular}{l|l|l|l|l|l|}
66 & 285 & 288 & 759
\end{tabular} \begin{tabular}{l|l|l|l|l|}
66 & 285 & 282 & 761
\end{tabular}

\begin{tabular}{l|l|l|l|l|l|}
666 & 285 & 276 & 763
\end{tabular}

\begin{tabular}{l|l|l|l|l|l|l|l|l|}
66 & 285 & 270 & 765
\end{tabular}

\begin{tabular}{l|l|l|l|l|l|}
66 & 285 & 2704 & 765 \\
66 & 283 & 329 & 745
\end{tabular}

\begin{tabular}{l|l|l|l|l|l|l|l|l|l|l|}
66 & 284 & 306 & 753
\end{tabular}

\begin{tabular}{l|l|l|l|l|}
66 & 285 & 253 & 771
\end{tabular} \begin{tabular}{l|l|l|l|l|l|l|l|l|l|l|l|l|l|l|}
666 & 282 & 194 & 791 \\
66 & 284 & 247 & 773
\end{tabular}

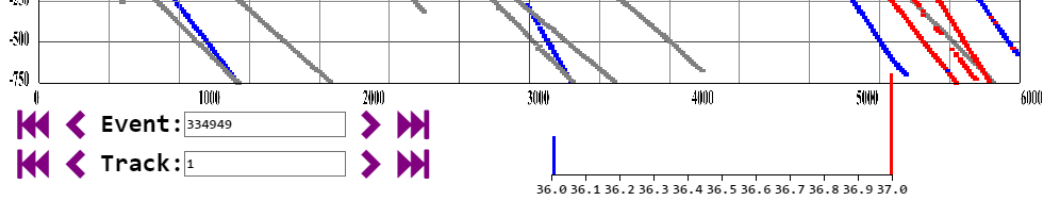

66 | $284 \mid 247$ | 773

Figure 4.4: DDT Event Display is used to compare tracks produced by the Moon shadow trigger before (grey tracks) and after (colored tracks) bug fix. 


\section{CHAPTER 5 OTHER}

This chapter includes information on work done at Fermilab, which is not related to the main topic of this thesis. Mainly this work is very technical and is more about fabrication and management of hardware/software than physics. At the same time, providing this work is an important part of my contribution to the Mu2e and NOvA experiments, so I think it's necessary to mention it shortly in this thesis.

\subsection{Mu2e: CSC production and testing}

As a particle detector, the CSC has a strong advantage over scintillators in their sensitivity to neutrons: CSC is about 2 orders of magnitude less sensitive to this type of background - see Fig. 5.1.

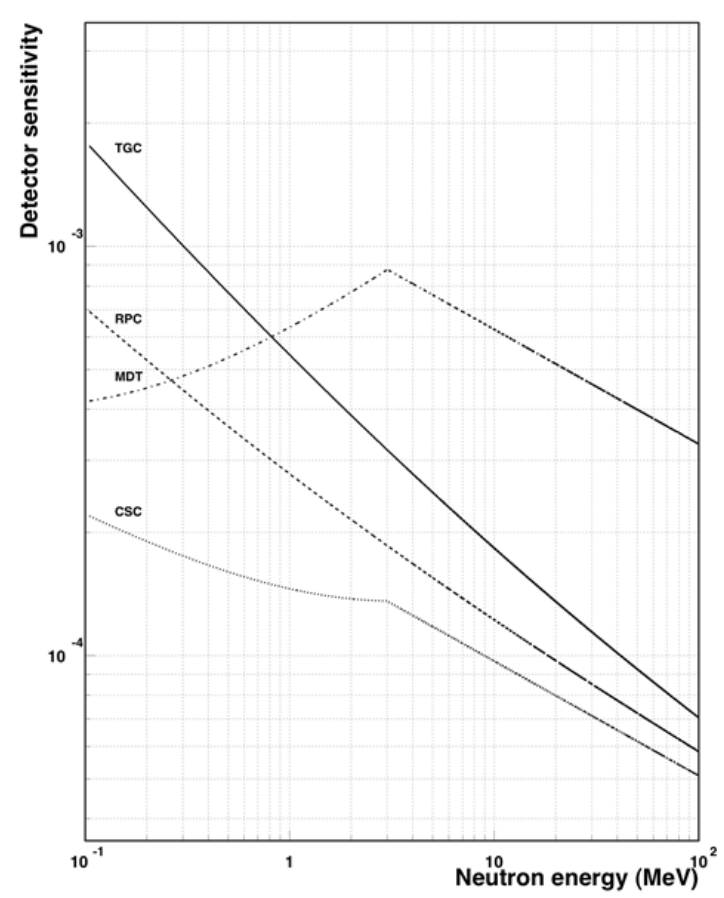

Figure 5.1: Sensitivity of different types of detectors (CSC and scintillators) to neutron background.

Four Cathode Strip Chambers (CSC) were produced at Fermilab in the summer 
of 2014 as a part of CRV system for Mu2e experiment. My contribution to this work was performing the final stages of production (applying capacitors and connectors, cleaning and sealing) and also testing the chambers (high voltage tests, performance, noise characteristics). Fabrication was done under supervision of O. Prokofiev (Fermilab) with major contribution from A. Artikov (JINR) and D. Chokheli (JINR). Also involved: Y. Oksuzian (UVA), C. Group (UVA), C. Dukes (UVA), Y. Wu (UVA) and, well, me.

The principle of how the CSC works can be described in the following way: 2 main elements form a wire chamber - a thin conductive wire and conductive strips. Wires and strips are charged with opposite sign and create a strong electric field between them. The volume between the wires and planes is filled with an insulating gas $\left(\mathrm{Ar} / \mathrm{CO}_{2}\right.$ mixture in case of these particular chambers). When a charged particle goes through the chamber it disturbs and polarizes the gas media. As a result, a current is registered at the outputs (signals are taken from both wires and strips). There are 2 sets of strips for each set of wires, which are located orthogonally to each other. Thus, taking signals from both sets of strips, one can locate the passing particle on a $2 \mathrm{D}$ plane.

The CSCs which were produced for Mu2e have 4 planes, each containing wires and 2 sets of strips. The anatomy of the produced CSCs is shown in Appendix $\mathrm{K}$.

The experimental setup is shown on Fig. 5.2 - it included oscilloscope, gas cylinder with $\mathrm{Ar} / \mathrm{CO}_{2}(80 \% / 20 \%)$ mixture, movable stand with crates and related devices (discriminators, gate generators, counters etc.), CSC, 2 scintillators above and below the CSC forming a cosmic telescope. The number of particles registered by telescope was compared to number of particles registered by CSC for efficiency and noise measurements.

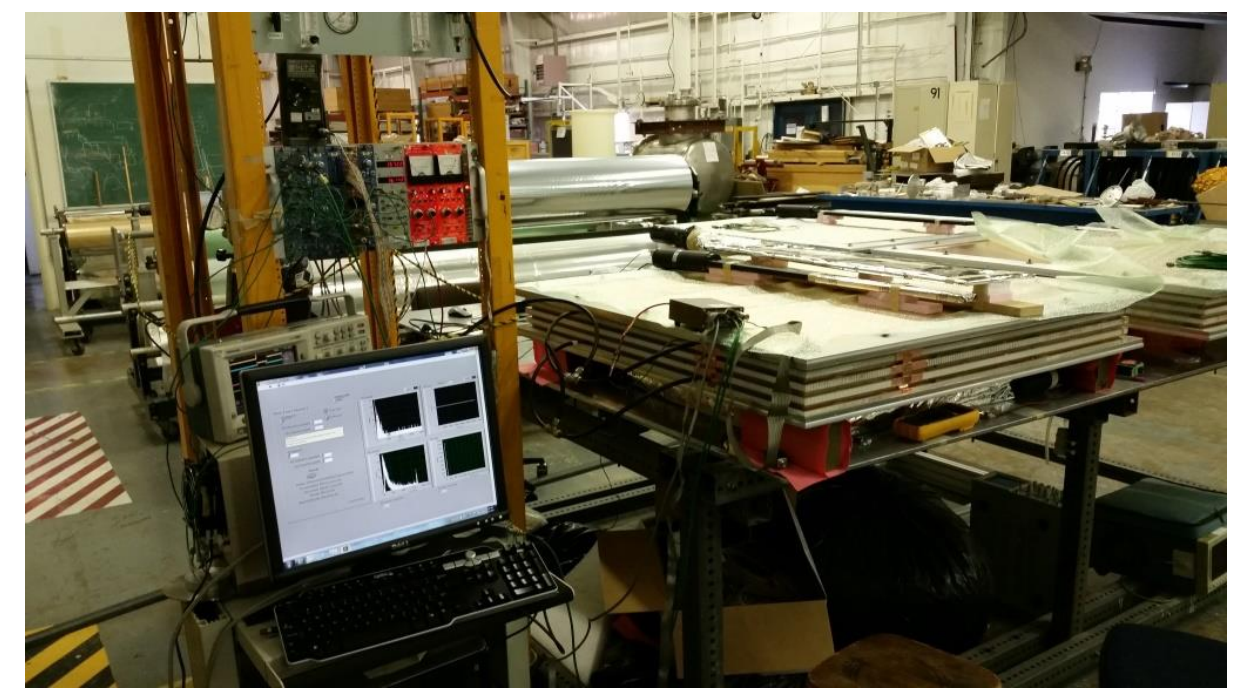

Figure 5.2: Experimental setup for testing CSC: CSC, gas cylinder, movable stand, oscilloscope, cosmic telescope consisting of 2 scintillators

With this setup, the following set of tests was performed: 
- High voltage (HV) tests:

- HV tests in air

- HV tests in $\mathrm{Ar} / \mathrm{CO}_{2}(80 \% / 20 \%)$ mixture

- Reverse polarity training

- HV tests in $\mathrm{Ar} / \mathrm{CO}_{2}(80 \% / 20 \%)$ mixture after training

- Test of CSC performance: taking signals from chamber

- Efficiency and noise test

- Signal amplitude and timing characteristics

HV tests are a measurement of electric current dependency on applied voltage (I-V curve). Since CSC, regarded as circuit, is not closed, current should be around 0 for all applied voltage values up to some critical voltage which produces sparking between anode wires and cathode strips. In realistic conditions the electric current can be nonzero for applied voltages lower than the critical limit due to impurities on the wire and cathode plane surfaces. These impurities create sources of small current. Significant current can point to more serious problems such as a short.

The result of the HV tests can be summarized in the following table of maximal working voltages with $\mathrm{Ar} / \mathrm{CO}_{2}(80 \% / 20 \%)$ mixture for each chamber:

\begin{tabular}{lllll}
\hline & Plane 1 & Plane 2 & Plane 3 & Plane 4 \\
\hline CSC \#1 & 3100 & 3200 & 3000 & 3150 \\
CSC \#2 & 3200 & 3220 & 3050 & 3220 \\
CSC \#3 & 3150 & 3200 & 3000 & 3200 \\
CSC \#4 & 3260 & 3280 & 3160 & 3240 \\
\hline
\end{tabular}

Table 5.1: Table of maximal working voltages (in $V$ ) for produced cathode strip chambers

The first signals were taken from one of the chambers (CSC1) with $\mathrm{Ar} / \mathrm{CO}_{2}$ $(80 \% / 20 \%)$ mixture under voltage of $3000 \mathrm{~V}$. Voltages were measured on both anode and cathode outputs. Received signals were self-triggered by signal from anode wires. See pictures of received signals on Fig. 5.3 .

Noise characteristics correspond to the total number of signals per second registered by the CSC. This number should be consistent throughout measurements of different planes and chambers. Test have shown such a consistency.

Efficiency is one of the main characteristics of the CSC. Since chambers were produced to detect passing charged particles, high efficiency is crucial for success of the project. Efficiency is measured as the ratio of registered signals to the total number of incident muons (where the latter is assumed to be the number of events registered by the 2-scintillator telescope system):

$$
E=\frac{N_{C S C}}{N_{\text {telescope }}}
$$




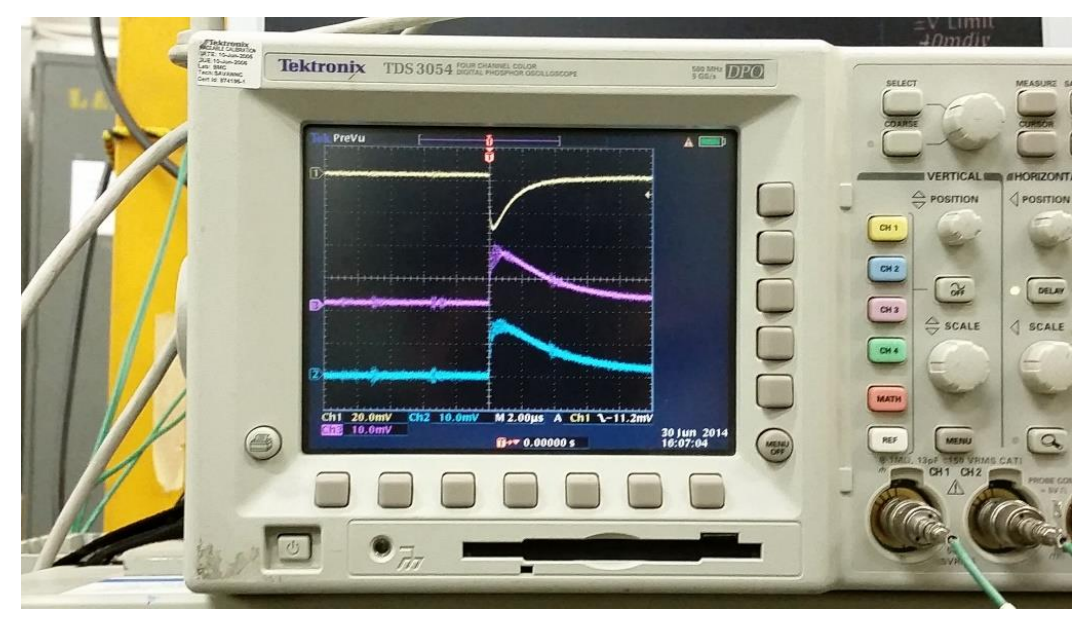

Figure 5.3: First signal from cosmic particles registered with CSC

Efficiency tests have shown that all layers of all chambers have good performance at peak voltages (which is close but usually about $100 \mathrm{~V}$ lower than maximal working voltage). The efficiency for each layer reaches $98-99 \%$.

More information on noise, efficiency and signal amplitude measurements is provided in Appendix $\mathrm{L}$.

Currently all 4 chambers are fully fabricated, assembled, tested and sealed. One of the chambers (CSC3) was sent to Brookhaven National Laboratory (BNL) for providing an additional set of tests targeted to applying readout system and taking signals using it. Other chambers reside in Fermilab's Lab6.

\subsection{Management for novasoft builds}

Another activity that I was involved in while at Fermilab is managing the NOvA DDT system releases keeping them in accordance with other parts of NOvA software. I took this responsibility from M. Frank (UVA postdoc at that time, now assistant professor at University of South Alabama). This management was especially active during the recent beam shutdown (beginning of August to beginning of November 2016), since this is the most appropriate time to make changes in software, update dependencies etc.

I managed the DDT system builds, kept track of changes of external dependencies such as NOvA DAQ release versions, and provided the DDT build for specific needs of the groups which take the DDT as a dependency (such as offline management group). The scheme of interdependencies of different NOvA software parts is shown in Fig. 5.4 .

Recently, I transferred my knowledge and responsibilities regarding the DDT management to Shih-Kai Lin (postdoc in University of Colorado). At the moment the whole management procedure is being changed towards a more transparent and manageable scheme with more strict definition of responsibilities. The build system will 

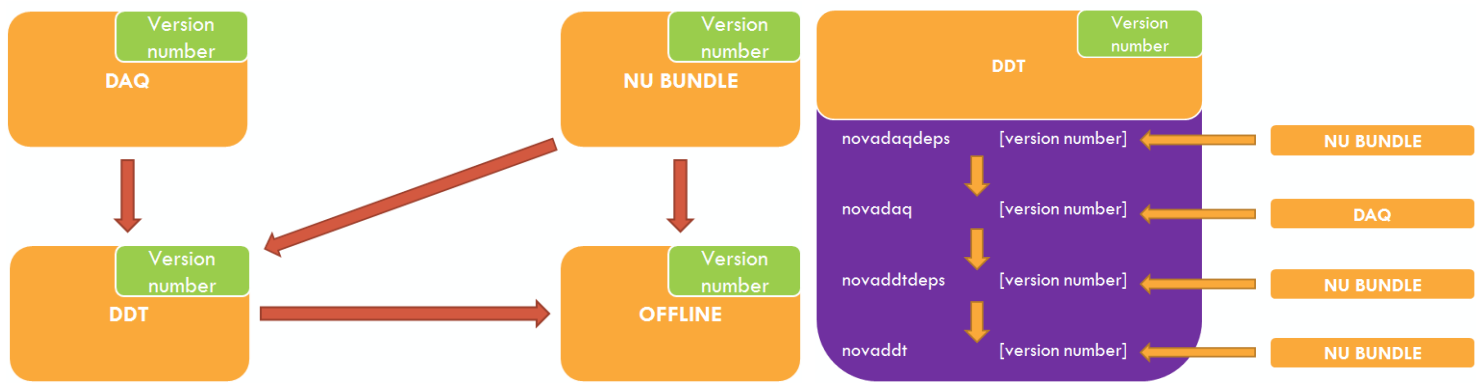

Figure 5.4: Scheme of dependencies of NOvA software

likely be changed too - from currently used MRB to SRT, since the latter is more common to other parts of the NOvA software. 


\section{Chapter 6 SUMMARY}

This thesis summarizes my work at UVA under the supervision of Prof. C. Group. Most of the material covered here can also be found in presentations and technical notes stored in the NOvA and Mu2e document databases. I hope that the tools and techniques which I helped develop for these experiments will be useful for future users, helping to facilitate data acquisition and analysis at NOvA and Mu2e.

Due to my efforts the Moon shadow trigger is now fully functional and taking data. It can still be adjusted to increase its efficiency and purity, but even with current configuration the rate of data coming from this trigger is several times larger than the rate which would have been taken with other existing trigger paths. The hope is to see the Moon shadow within several months of data acquisition.

The upward-going muon trigger is also stable and continuously collects data. Due to its exciting prospects, the upward-going muon analysis recently attracted attention of the collaboration and new members (Leonidas Aliaga, Aristeidis Tsaris and Andrew Norman) have become involved. We hope to use this unique dataset to search for dark matter. With a collaborative effort we may also adopt interesting machine learning techniques to facilitate and improve the sensitivity of our analysis and provide more complex metrics of track directionality.

The 3D event display I created should be a useful tool for some visualization challenges, such as comparing tracks reconstructed offline and tracks reconstructed at the trigger level. It already proved helpful when trying to understanding the track differences and hit composition for different tracking algorithms. The 3D option of this event display can be useful for such studies as the Moon shadow analysis, analysis of cosmic showers, etc.

The work I did on the cathode strip chambers was a significant contribution to the Mu2e experiment. The cathode strips will be an important tool for testing the performance of the cosmic ray veto system of the Mu2e experiment.

Its exciting to think that the work I have done can continue to benefit NOvA and Mu2e for years beyond my graduation. 


\section{APPENDix A Neutrino Oscillation Parameters}

Current estimates for neutrino oscillation parameters are taken from Particle Data Group, 2015 [11]. Here NH, RH stand for normal mass hierarchy and reversed mass hierarchy correspondingly, $\Delta m^{2}=m_{3}^{2}-\left(m_{1}^{2}+m_{2}^{2}\right) / 2$.

\begin{tabular}{ll}
\hline Parameter & \multicolumn{1}{c}{ Best fit $(1 \sigma)$} \\
\hline$\Delta m_{12}^{2}\left[10^{-5} e V^{2}\right]$ & $7.54_{-0.22}^{+0.26}$ \\
$\left|\Delta m^{2}\right|\left[10^{-3} e V^{2}\right], \mathrm{NH}$ & $2.43_{-0.06}^{+0.06}$ \\
$\left|\Delta m^{2}\right|\left[10^{-3} e V^{2}\right], \mathrm{RH}$ & $\left(2.38_{-0.06}^{+0.06}\right)$ \\
$\sin ^{2}\left(\theta_{12}\right)$ & $\left(0.308_{-0.017}^{+0.017}\right)$ \\
$\sin ^{2}\left(\theta_{23}\right), \mathrm{NH}$ & $\left(0.437_{-0.023}^{+0.033}\right)$ \\
$\sin ^{2}\left(\theta_{23}\right), \mathrm{RH}$ & $\left(0.455_{-0.031}^{+0.039}\right)$ \\
$\sin ^{2}\left(\theta_{13}\right), \mathrm{NH}$ & $\left(0.0234_{-0.0019}^{+0.0020}\right)$ \\
$\sin ^{2}\left(\theta_{13}\right), \mathrm{RH}$ & $\left(0.0240_{-0.0022}^{+0.0019}\right)$ \\
$\delta_{C P} / \pi, \mathrm{NH}$ & $\left(1.39_{-0.27}^{+0.38}\right)$ \\
$\delta_{C P} / \pi, \mathrm{RH}$ & $\left(1.31_{-0.33}^{+0.29}\right)$ \\
\hline
\end{tabular}

Table A.1: Current estimates for parameters of neutrino oscillations 


\section{ApPendix B NOvA FD Exposure AND Volume}

Plots here represent the effective acceptance and the fiducial volume of the NOvA Far Detector versus incident particle angles with various cuts on a track length. Here azimuthal angle 0 points to North, zenith is the angle between an incident particle and a vertical axis.

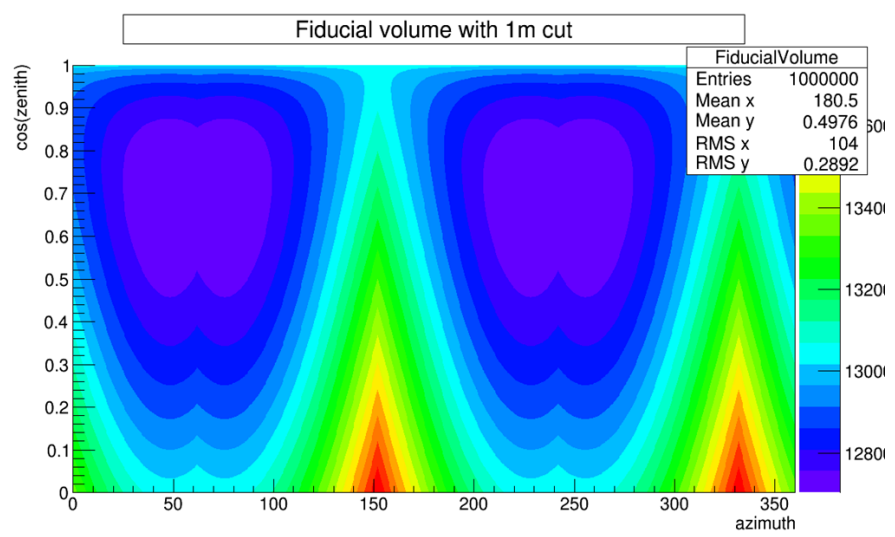

Figure B.1: Fiducial volume of the Far Detector with 1 m cut. Values are in $m^{3}$

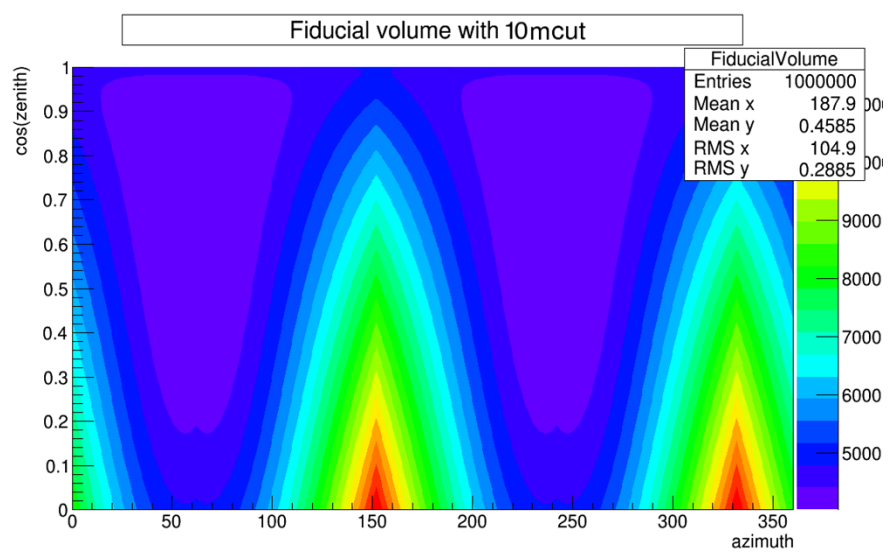

Figure B.2: Fiducial volume of the Far Detector with $10 \mathrm{~m}$ cut. Values are in $m^{3}$

As a cross-check of data sensibility, the histogram of the relation between the 


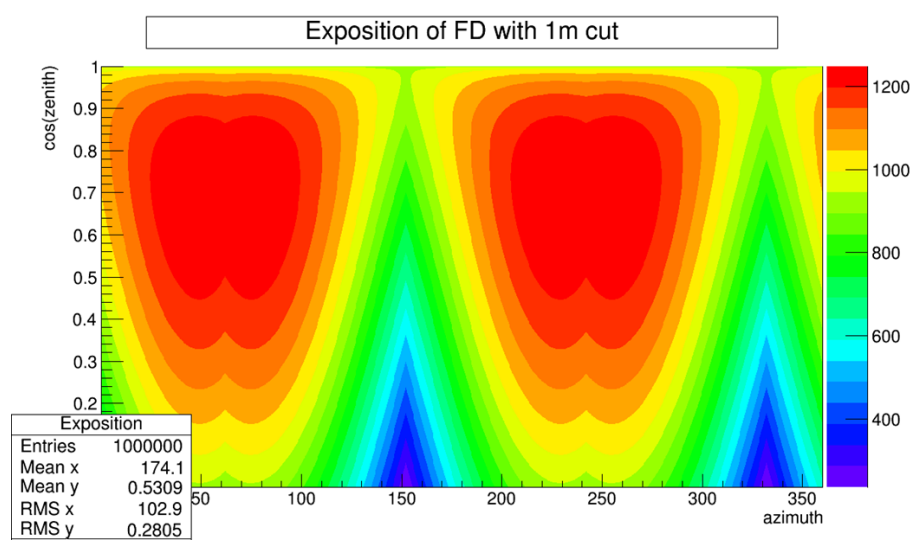

Figure B.3: Effective acceptance of the Far Detector with $1 \mathrm{~m}$ cut. Values are in $m^{2}$

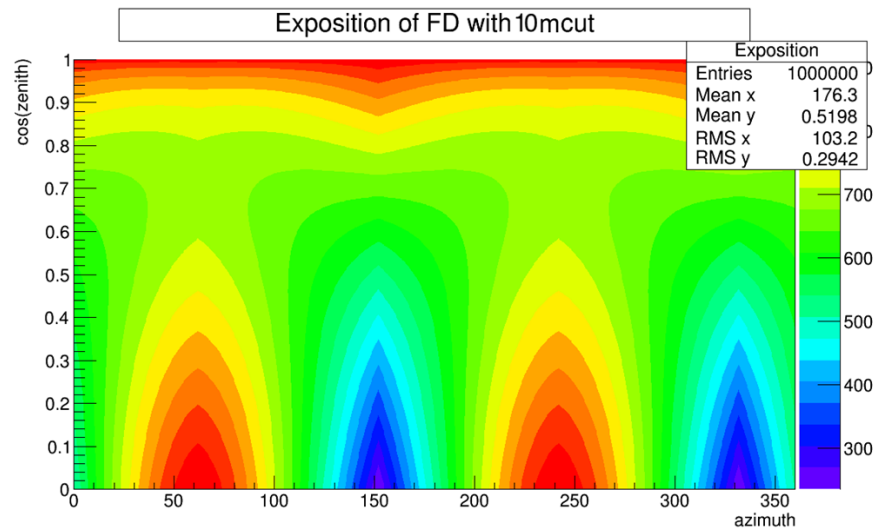

Figure B.4: Effective acceptance of the Far Detector with $10 \mathrm{~m}$ cut. Values are in $m^{2}$

track length and the number of hits in a track was made. It shows that this relation seems reasonable (see Fig. B.5). 


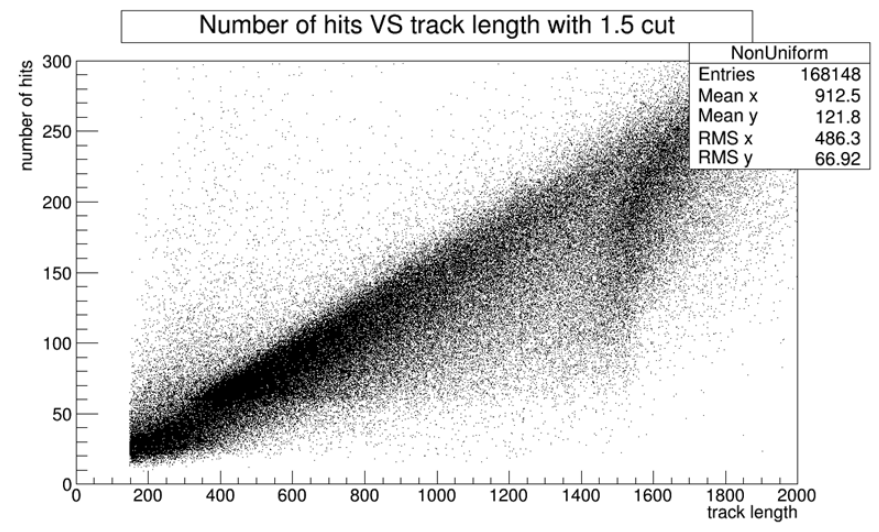

Figure B.5: Track length in $\mathrm{cm}$ versus track number of hits in a track. The data is taken from cosmic pulser file. 


\section{Appendix C Moon Trigger Rates}

Sample plots of Moon trigger rates from the NOvA online monitoring system Ganglia are presented in Fig. C.1 C.2. The combined plot for the Moon and the Sun rates shows that total rate doesn't exceed $20 \mathrm{~Hz}$ - see Fig. C.3.

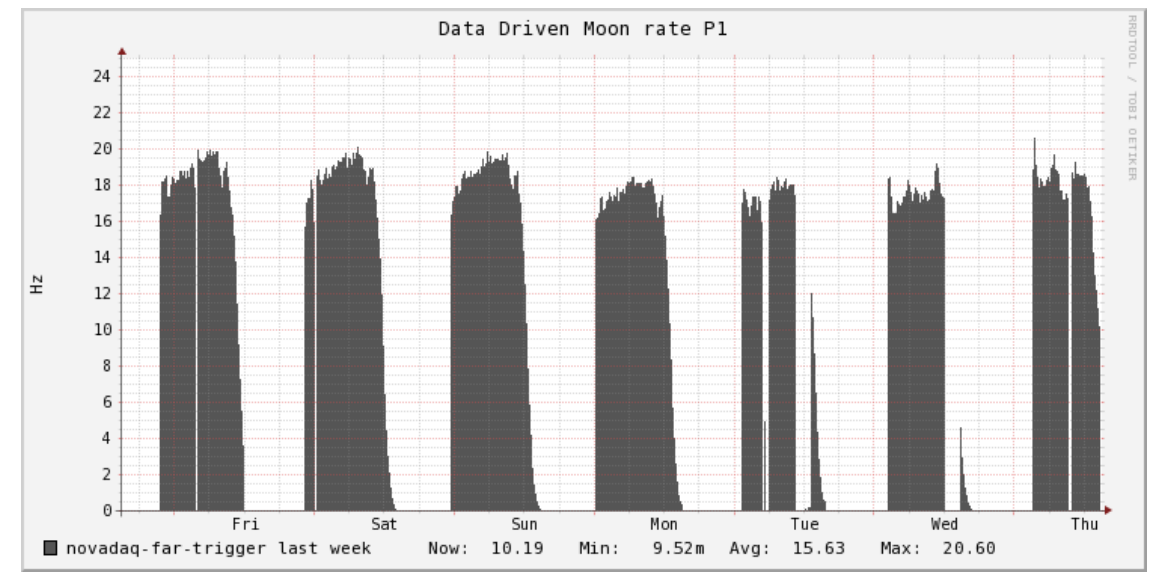

Figure C.1: Moon trigger rate plot taken from Ganglia

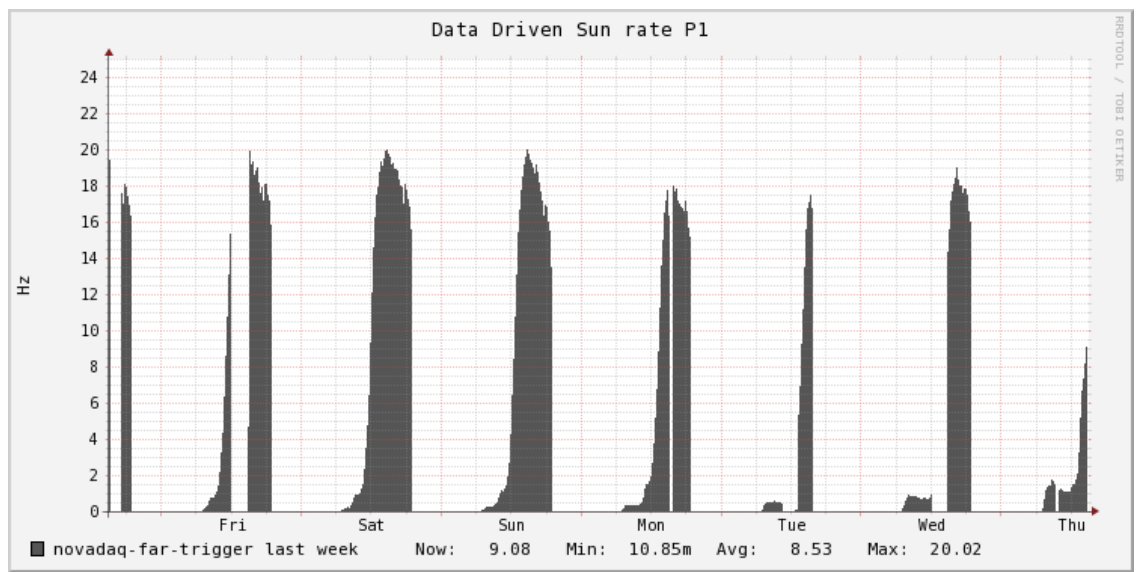

Figure C.2: Sun trigger rate plot taken from Ganglia 


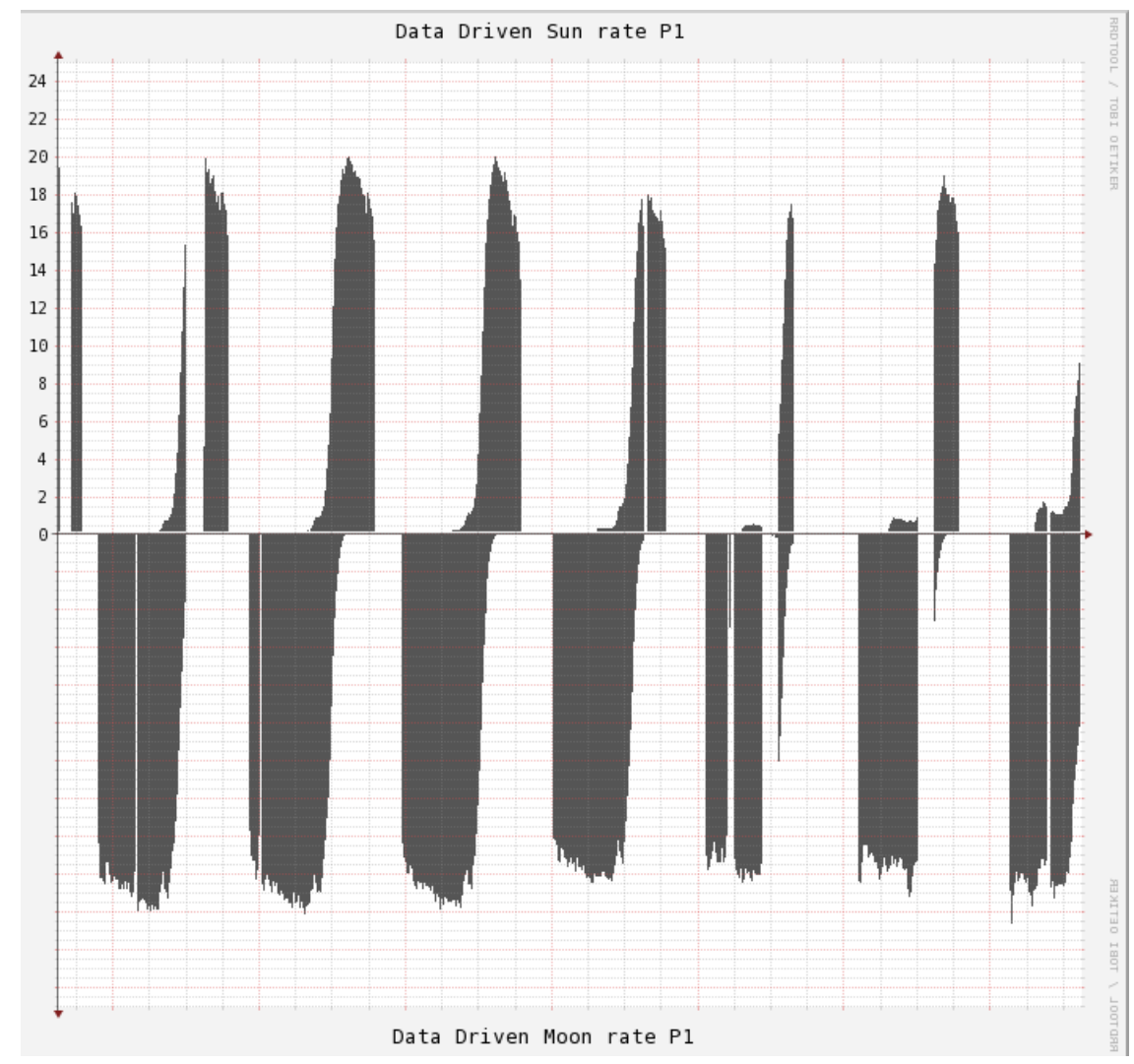

Figure C.3: Sun and Moon trigger rate combined, separate plots are taken from Ganglia 


\section{Appendix D NOVAS and Celestial Locator Code}

The underlying library for defining position of celestial bodies - NOVAS - has following functions which are principal for our task of locating the Moon and the Sun at some particular geographical position. Main functions of NOVAS for our purposes are: ephem_open("FILE_NAME" , \&jd_beg, \&jd_end, \&de_num;- opens ephemerides file. Outputs are minimal and maximal Julian dates and ephemerides file version (DExxx)

make_on_surface (latitude, longitude, height, temperature,pressure, \&geo_loc - this makes point on surface of the Earth

make_cat_entry ("DUMMY", "xxx" ,0,0.0,0.0,0.0,0.0,0.0,0.0,\&dummy_star) ; - make a dummy object which later will be used to generate the Sun and the Moon objects error = make_object $(0,11$, "Moon", \&dummy_star, \&moon); - generate the Moon and the Sun objects from a dummy object

error = make_object $(0,10$, "Sun", \&dummy_star, \&sun);

error = topo_planet (jd_tt, \&obj, delta_t, \&geo_loc, accuracy, \&rat, \&dect, \&dist); - gets topocentric coordinates of celestial body, i.e. coordinates in a coordinate system having its center in the center of the Earth taking axes with respect to given coordinates

equ2hor(jd_ut1, delta_t, accuracy, 0, 0, \&fGeoLoc, rat, dect, 0, \&zen, \&azi, \&rar, \&decr); - gets elevation and azimuthal angles of a given celestial body in a given point on the surface of the Earth

You can use this service in following way:

First, when you create or modify your module don't forget to include a reference to the module:

\#include "CelestialLocator/CelestialLocator.h"

Second, modify your gnumake file and xxxjob.fcl of your module adding a reference to the CelestialLocator service as shown in this example:

override LIBLIBS+=-L $\$\left(A R T \_L I B\right) \ldots$-lCelestialLocator_service ....

Third, modify your fcl so it uses the service:

\#include "CelestialLocator.fcl"

Also add a reference to the CelestialLocator service in services section of your fcl: 
CelestialLocator: @local: :standard_celestial

Finally, use locator in your code - first, define a variable of the service inside your module class or outside of it:

art: : ServiceHandle<locator: :CelestialLocator> loc;

Then use its functions ( $t$ here is the Unix time, zen and azi are defined as references somewhere before calling this function):

loc->GetMoonPosition_FD(t, zen, azi);

The zenith and azimuthal angles of the Moon at the FD position for specified time will be stored in a zen and azi variables respectively. 


\section{Appendix E Moon Trigger Run History}

Moon trigger stages and history:

- 8 Jul 2015: trigger is brought online.

Trigger wasn't taking data due to error

Run 19996

- 11 Aug 2015: trigger starts taking data.

Prescale is set to 1000

AngularCut is set to 5 degrees

Track length cut is set to $10 \mathrm{~m}$

Run 20270

- 2 Sep 2015: new track length and hit number cut are introduced (this configuration had error, so this data isn't good).

Prescale is set to 1000

AngularCut is set to 5 degrees

Track length cut is set to $1.5 \mathrm{~m}$

Hit number cut is set to 10

Run 20414

- 10 Sep 2015: error in previously released trigger was found and fixed

Prescale is set to 1000

AngularCut is set to 5 degrees

Track length cut is set to $1.5 \mathrm{~m}$

Hit number cut is set to 10

Run 20464

- 1 Oct 2015: smart prescale is introduced Prescale is set to 1

AngularCut is set to 5 degrees

Track length cut is set to $1.5 \mathrm{~m}$

Hit number cut is set to 10

Average rate is set to $20 \mathrm{~Hz}$

Angular suppression parameter $\sigma$ is set to 10

Run 20666

- 2 Feb 2016: change in dynamic prescale with a correction according to a new gain Prescale is set to 1

AngularCut is set to 5 degrees 
Track length cut is set to $1.5 \mathrm{~m}$

Hit number cut is set to 10

Average rate is set to $16 \mathrm{~Hz}$

Angular suppression parameter $\sigma$ is set to 5

New prescale lookup table is introduced

Run 22157

- 30 Aug 2016: fixing a bug in the trigger code All parameters stay the same New prescale lookup table is introduced

Run 23941 


\section{APPENDIX F Upward Going Muons Rate Estimate}

To know the expected rate of registration of upward-going atmospheric neutrinos in the NOvA Far Detector, we made a simplistic calculation based on the PDG data [11, known muon energy deposition, known size and material composition of the detector. Providing this calculation we used these simplifications, which are explained later in the text:

- All cosmic rays are protons

- Flux of muon and electron neutrino is the same at ground level

- Flux of neutrinos is uniform across the sky

- All neutrinos have an energy of $1 \mathrm{GeV}$

- No matter effect in neutrino oscillations is taken into account

- Muon neutrino can oscillate into electron and tau neutrinos, electron neutrinos can oscillate into muon neutrinos only

- We don't account for tau neutrinos in detector

- We don't account for different fiducial detector volume seen from different angles

- We account only for muons coming from the rock, neglecting electrons

According to the PDG, the most common constituent of cosmic rays are protons. We can roughly estimate cosmic ray energy as $1-2 \mathrm{GeV}$, this is where most of protons from cosmic rays reside. Also, we can roughly estimate rate of $\nu_{e}, \nu_{\mu}$ the same at the ground level (due to oscillations in contrast to $2: 1$ ratio without it). $\nu_{\mu}$ rate at ground level can be taken as (again from the PDG):

$$
F_{\nu_{\mu}} \approx 110 m^{-2} s^{-1} s r^{-1}
$$

Neutrino flux peaks at $E_{\nu} \approx 1 G e V[25]$. For this energy we can consider only muon and electron neutrino interaction events that are detectable, since tau neutrino should have more energy to be detected (due to high mass of $\tau$-lepton).

We can neglect transition between electron and tau neutrino and estimate the oscillation probability as follows: 
For $\nu_{\mu} \rightarrow \nu_{e}$ transition we have 3 -flavor oscillation formula (not accounting for the MSW effect):

$$
P_{\nu_{\mu} \rightarrow \nu_{e}}=P_{\nu_{e} \rightarrow \nu_{\mu}}=\sin ^{2}\left(\theta_{23}\right) \sin ^{2}\left(2 \theta_{13}\right) \sin ^{2}\left(1.267 \frac{\Delta m_{31}^{2}\left(e V^{2}\right) L(k m)}{E(G e V)}\right)
$$

For $\nu_{\mu} \rightarrow \nu_{\tau}$ we can use 2-flavor oscillation formula [26] (not regarding MSW effect):

$$
P_{\nu_{\mu} \rightarrow \nu_{\tau}}=\sin ^{2}\left(2 \theta_{23}\right) \sin ^{2}\left(1.267 \frac{\Delta m_{32}^{2}\left(e V^{2}\right) L(k m)}{E(G e V)}\right)
$$

Assuming oscillation parameters for Normal Hierarchy $\left(m_{1}<m_{2}<m_{3}\right)$ from Appendix $\mathrm{A}$ and disregarding uncertainties we get:

$$
\begin{aligned}
& P_{\nu_{\mu} \rightarrow \nu_{e}}=P_{\nu_{e} \rightarrow \nu_{\mu}}=0.04 \sin ^{2}\left(3.56 \frac{L(1000 \mathrm{~km})}{E(G e V)}\right) \\
& P_{\nu_{\mu} \rightarrow \nu_{\tau}}=0.984 \sin ^{2}\left(3.46 \frac{L(1000 \mathrm{~km})}{E(\mathrm{GeV})}\right)
\end{aligned}
$$

Using this simple approximation we can find angular distribution of the neutrino flux. And here we use another simple approximation that the flux is uniform across the sky, which is not really true, since we know that really highly energetic neutrino rate is higher closer to horizon than near zenith.

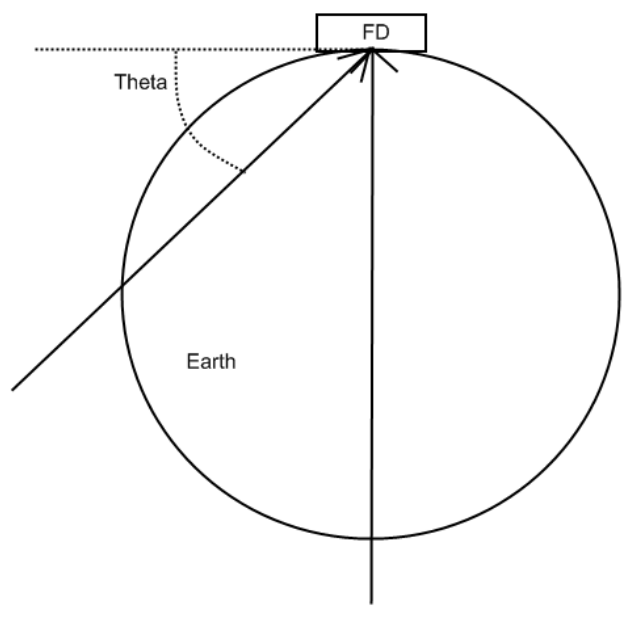

Figure F.1: Neutrino paths to NOvA Far Detector.

Distance which neutrino travels through the Earth is $L=2 R_{\odot} \cos (\pi / 2-\theta)=$ $12800 \cos (\pi / 2-\theta) \mathrm{km}$. Thus, rate of $\nu_{e}$ is determined as (see Fig. F.2):

$$
\begin{gathered}
F_{\nu_{e}}(\theta)=110 \mathrm{~m}^{-2} \mathrm{~s}^{-1} s r^{-1} \\
F_{\nu_{\mu}}(\theta)=110\left(1-0.984 \sin ^{2}(44.28 \cos (\pi / 2-\theta))\right) m^{-2} s^{-1} s r^{-1}
\end{gathered}
$$




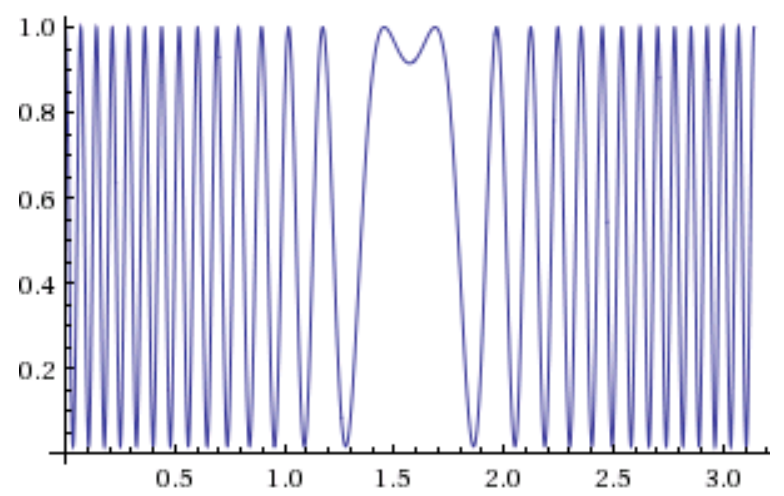

Figure F.2: Muon neutrino flux at NOvA FD depending of angle to horizon, values of angle in radians, values of flux are arbitrary

To estimate event rate in the detector (contained-vertex) we need to integrate this value over stereo angle $d \Omega=2 \pi \sin (\theta) d \theta$. Also we need to know neutrino cross section at $1 \mathrm{GeV}$ (taken from PDG) and number of nucleons $N_{N}$ in the detector. Number of nucleons is derived from the mass of the detector (14kT for FD), since we can neglect the mass of electrons there.

We also need to take into account the rate of rock muons - see Fig. F.3. We can neglect electrons coming from the electronic neutrino interaction in the surrounding rock, since electronic showers are short enough, so they will not be registered in the detector.

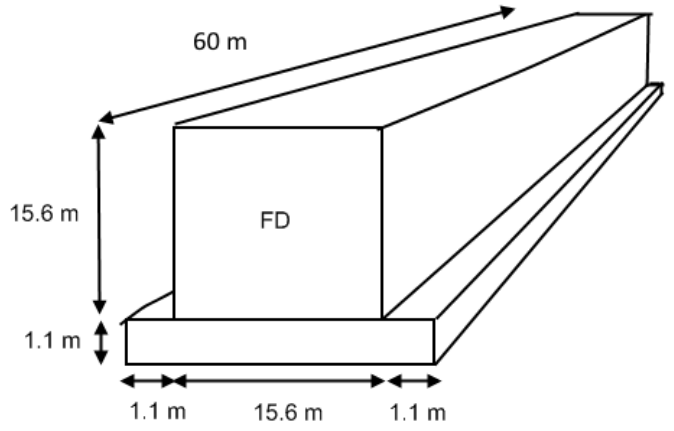

Figure F.3: The NOvA FD with surrounding rock

We estimate the muon mean energy as $0.5 E_{\nu} \approx 0.5 \mathrm{GeV}$. For standard rock we can take $\rho=2.65 \mathrm{~g} / \mathrm{cm},\left\langle\frac{d E}{d x}\right\rangle=1.688 \mathrm{MeV} \mathrm{cm}^{2} / \mathrm{g}$ [27]. Thus, mean path of muon would be $E_{\mu} /\left(\left\langle\frac{d E}{d x}\right\rangle \cdot \rho\right) \approx 1.1 \mathrm{~m}$. The effective mass of the surrounding rock producing muons then is: $\rho \cdot 1.1 \cdot(15.6+2) \cdot(78+2) \approx 4.2 \mathrm{kT}$. 
Making these assumptions we get:

$$
\begin{aligned}
& N_{N}^{\mu}=N_{N}^{\text {det }}+N_{N}^{\text {rock }} \approx 1.1 \times 10^{34} \\
& N_{N}^{e}=N_{N}^{\text {det }} \approx 8.36 \times 10^{33} \\
& P_{N C, \nu_{\mu}}=\sigma_{N C} N_{N}^{\mu} \int_{\text {half sphere }} F_{\nu_{\mu}} d \Omega \\
& P_{C C, \nu_{\mu}}=\sigma_{C C} N_{N}^{\mu} \int_{\text {half sphere }} F_{\nu_{\mu}} d \Omega \\
& P_{N C, \nu_{e}}=\sigma_{N C} N_{N}^{e} \int_{\text {half sphere }} F_{\nu_{e}} d \Omega \\
& P_{C C, \nu_{e}}=\sigma_{C C} N_{N}^{e} \int_{\text {half sphere }} F_{\nu_{e}} d \Omega
\end{aligned}
$$

where $N_{N}$ is number of nucleons, $N C$ and $C C$ stand for neutral current interactions and charged current interactions respectively, $\sigma$ is the neutrino interaction crosssections, $F$ is the neutrino flux and $P$ is the probability of an interaction. For $C C$ interactions with muon production the total cross section for the muon neutrino scattering (for $1 \mathrm{GeV}$ neutrino) is $\sigma_{C C} \approx 0.7 \times 10^{-38} \mathrm{~cm}^{2} /$ nucleon.

As a result, using these simplified consideration we got the following estimate for the event rate:

$$
\begin{aligned}
& P_{C C, \nu_{e}}=0.0404 \times 10^{-4} \mathrm{~Hz} \quad(0.35 / \text { day }) \\
& P_{C C, \nu_{\mu}}=0.028 \times 10^{-4} \mathrm{~Hz} \quad(0.3 / \text { day })
\end{aligned}
$$

One should note that this is a pretty rough estimation. Nonetheless, comparing it to experimental results, we can conclude that this estimate proves to be reasonable: Super-Kamiokande collaboration have registered 614 up-going muons during 537 detector live days [28]. They have $22 k T$ of fiducial volume. Neglecting effects of a difference in magnetic fields, we should register (assuming we have $14 k T$ of fiducial volume) about 0.72 up-going muons a day, which is in a good agreement with the simplistic calculation we made. 


\section{APPENDIX G DDT EFFICIENCY AND PURITY}

The NOvA DDT system uses Hough tracker algorithm to reconstruct tracks online. The offline analysis can use variety of trackers: window tracker, cosmic tracker or Kalman tracker. The latter is used for specific needs and reconstructs track under very special conditions of a track location and direction, so practically for analysis like upward-going muons search or the Moon shadow analysis we use window or cosmic trackers. The offline reconstruction is not restricted in time and computer resources, so generally it is more accurate.

We checked how the DDT system reconstructs tracks and compared it with offline track reconstruction as well as with views of a tracks in the offline event display (it uses raw data converted into ROOT format). It turned out that missing tracks in the DDT system is a quite regular process. Another regular type misreconstruction in the DDT system is track duplicates: some tracks repeat several times in the same event, sometimes having slightly different coordinates. In these studies, tracks were identified as duplicates if their start and end points were located closer than $1.5 \mathrm{~m}$.

Example of duplicated tracks is provided on Fig. G.1 (there first 3 numbers at each row are $x, y, z$ coordinates of the start point of the track and numbers on the right are corresponding coordinates of the end point in cell and plane indices).

In this analysis the following definitions of efficiency and purity were introduced:

$$
\begin{aligned}
E & =\frac{\# \text { of }(D D T \text { \& reco }) \text { tracks }}{\# \text { of reco tracks }} \\
P & =\frac{\# \text { of }(D D T \& \text { reco }) \text { tracks }}{\# \text { of DDT tracks }}
\end{aligned}
$$

Plots G.2 G.4 and table G.1 summarize findings of the DDT efficiency and purity with the use of different trackers for the offline reconstruction process.

\begin{tabular}{lll}
\hline Tracker & DDT efficiency & DDT purity \\
\hline Cosmic tracker & $84.6 \%$ & $82.0 \%$ \\
Window tracker & $86.7 \%$ & $82.5 \%$ \\
Kalman tracker & $82.6 \%$ & $0.06 \%$ \\
\hline
\end{tabular}

Table G.1: The DDT system efficiency of the track reconstruction when compared to different offline reconstruction algorithms.

To illustrate misreconstruction processes in the DDT and offline systems, we used combination of the offline event display (it doesn't use any reconstruction process 


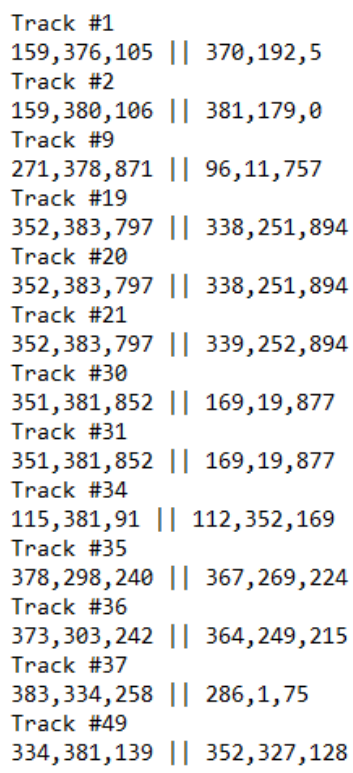

Figure G.1: Example of duplicated tracks in the DDT system (duplicates are combined into groups and marked by the same color). Coordinates are given in cell and plane indices.
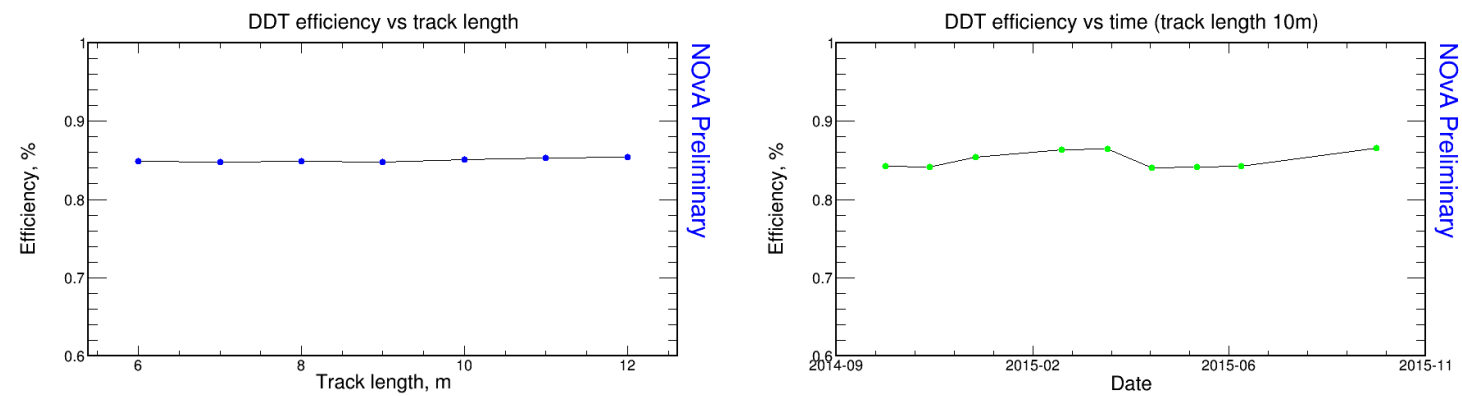

Figure G.2: The DDT system efficiency VS track length (left) and time (right). The cosmic tracker is used for comparison with DDT.

when displaying hits, so we can count it as ground truth) and developed 3D event display (see Chapter 4). Pictures taken for some particular event (cosmic pulser data from the NOvA FD: run 19539, subrun 32, event 480534) are shown on Fig. G.5.G.8.

Overall, conclusion about efficiency and purity of DDT tracking system is that the efficiency is about $85 \%$ and stays more or less constant with time and track length. The purity of the DDT system (with duplicate tracks removed) is around $80 \%$ and grows with track length and doesn't depend on time. The purity of the DDT system (taking into account duplicate tracks) behaves similarly, but stays at the level of $60 \%$. 

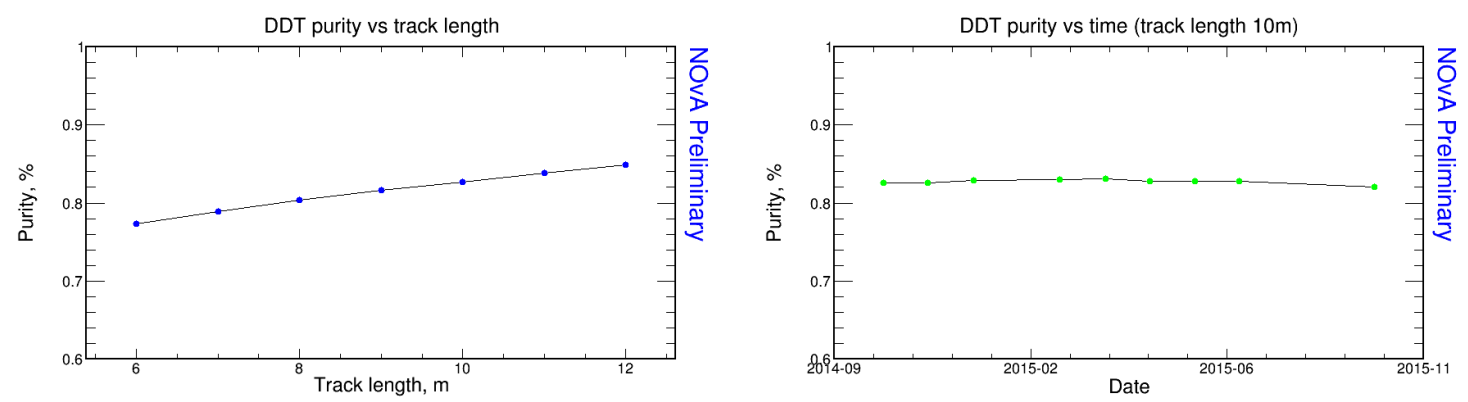

Figure G.3: The DDT system purity VS track length (left) and time (right). The cosmic tracker is used for comparison with DDT.
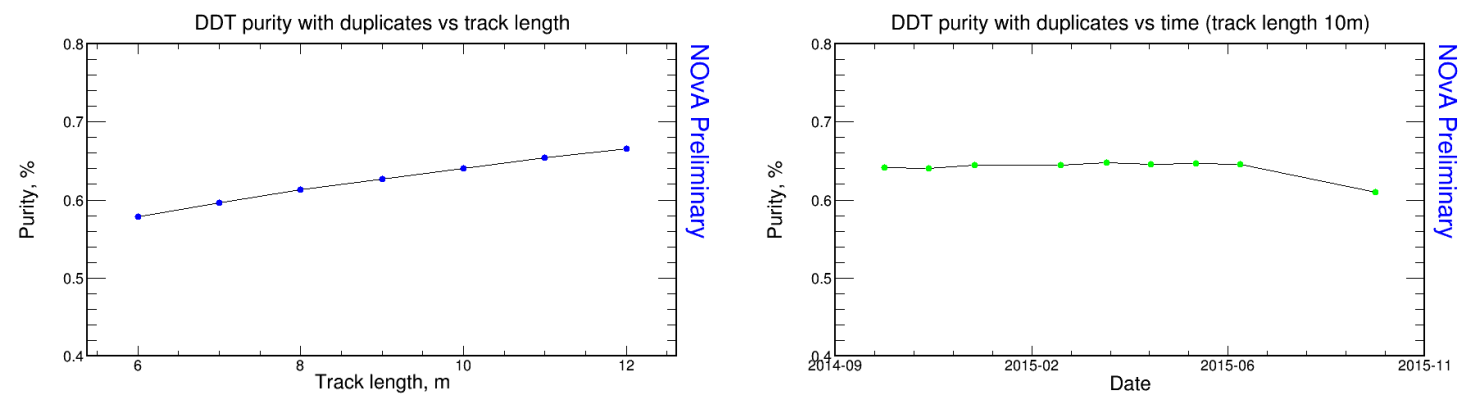

Figure G.4: The DDT system purity including duplicate DDT tracks

VS track length (left) and time (right). The cosmic tracker is used for comparison with the DDT.

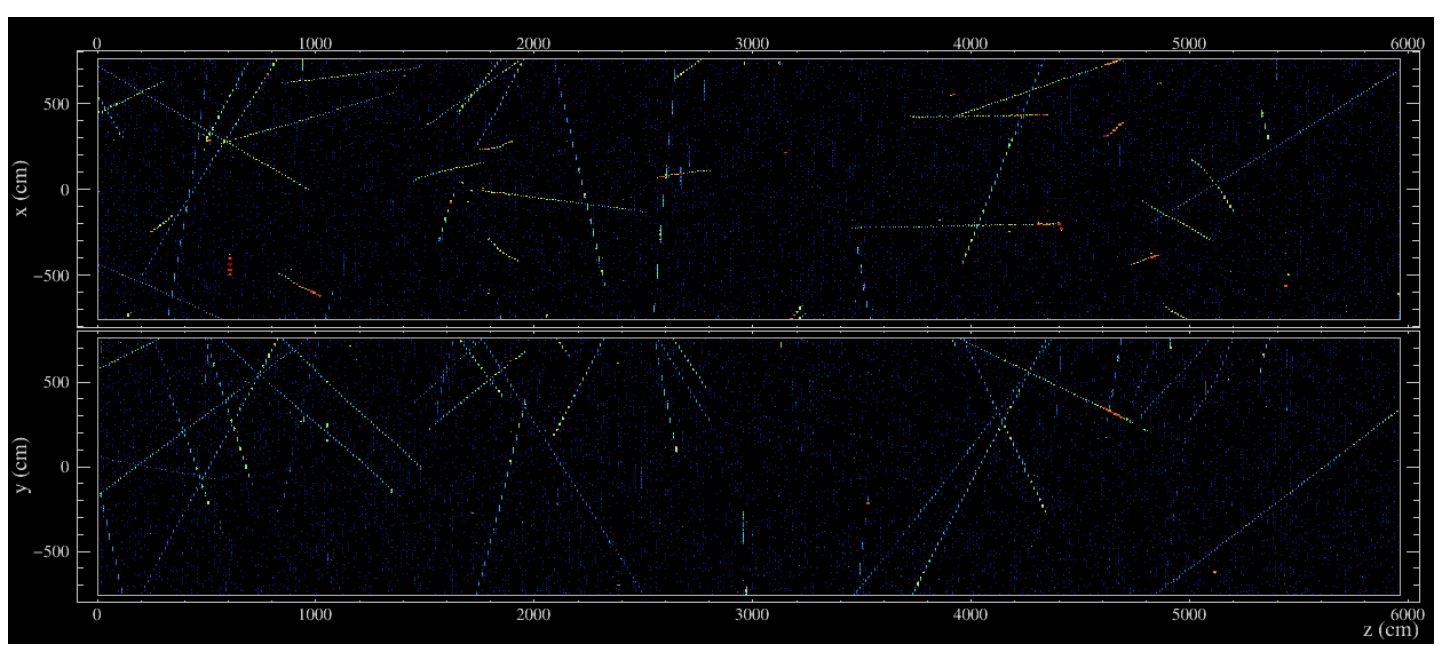

Figure G.5: Tracks in the offline event display for a cosmic pulser file, run 19429, subrun 32, event 480476 . 


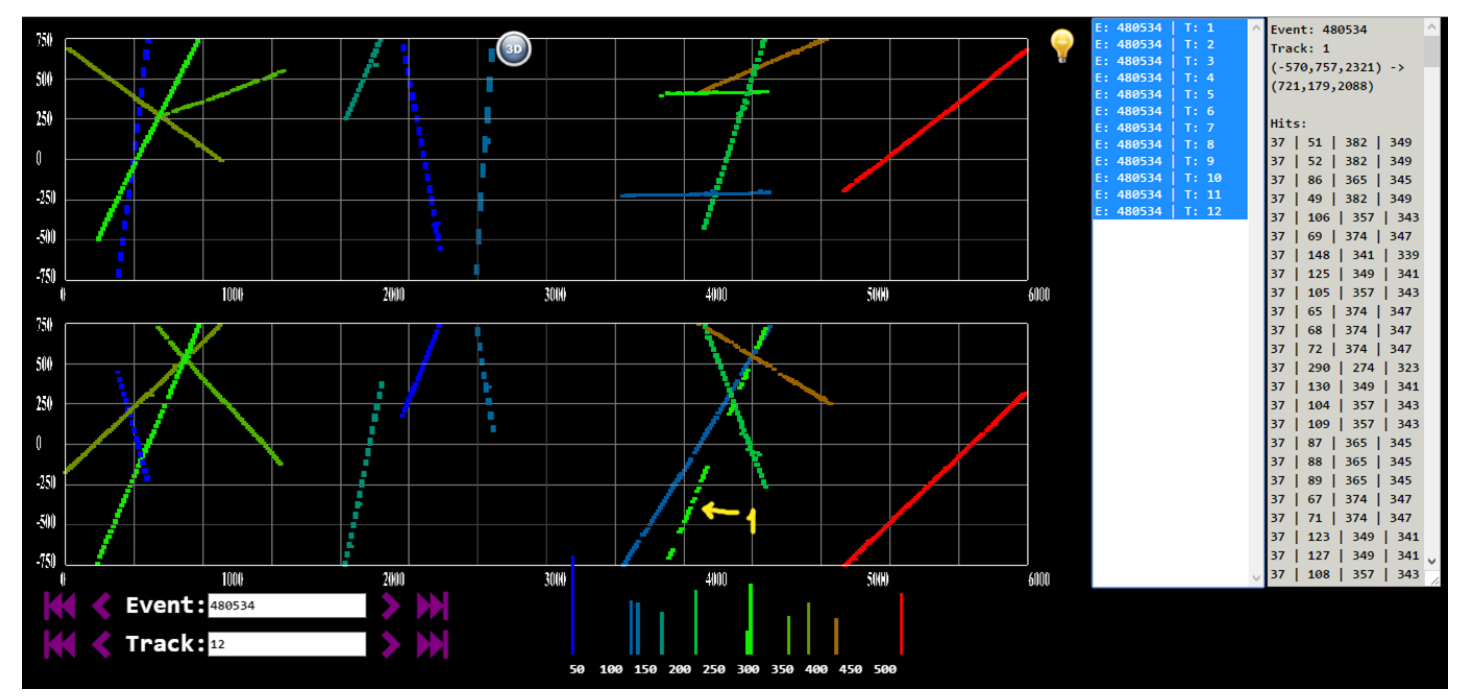

Figure G.6: Tracks (DDT) in the 3D event display for cosmic a pulser file, run 19429, subrun 32, event 480476.

1: poorly reconstructed track

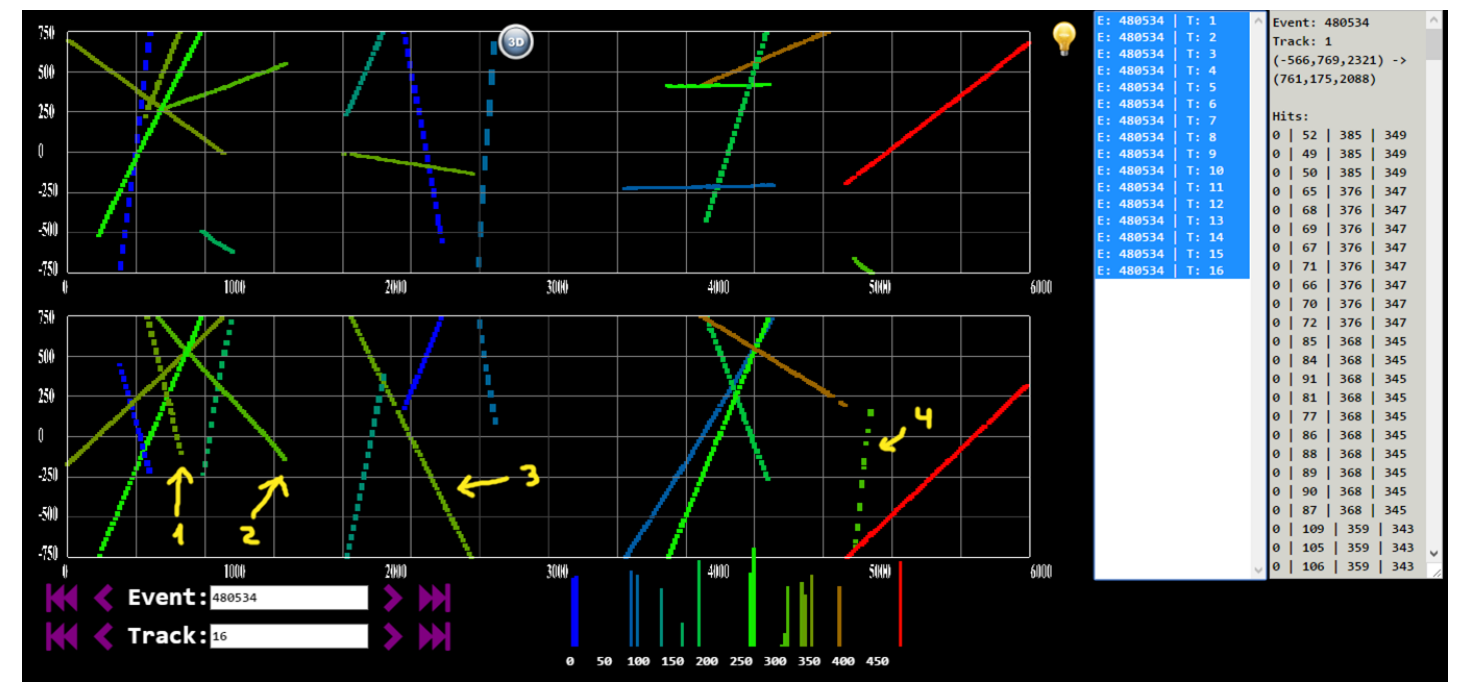

Figure G.7: Tracks (cosmic tracker) in the 3D event display for a cosmic pulser file, run 19429, subrun 32, event 480476.

1,2,3: tracks are absent in the DDT

4: track is absent in both DDT and window tracker 


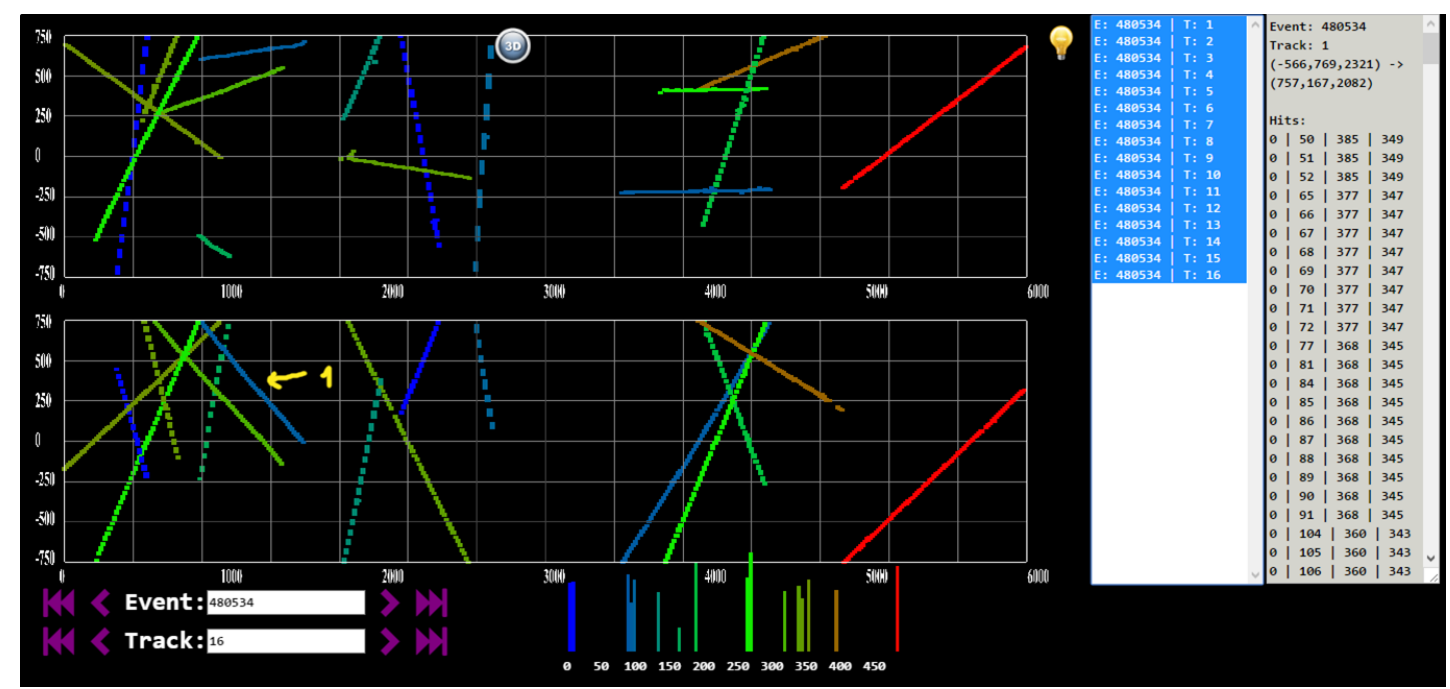

Figure G.8: Tracks (window tracker) in the 3D event display for a cosmic pulser file, run 19429, subrun 32, event 480476.

1: track is absent in both DDT and cosmic tracker 


\section{APPENDIX H UpMu Data Quality}

We perform several checks on the data quality to ensure consistent performance in our data and to exclude data files with obvious anomalous properties. We compare mean track properties for all of the subruns included in the analysis. Deviations from the normal of these parameters could be an indicator of malfunction of detector systems, data acquisition software, reconstruction algorithms or some combination of those factors. We exclude anomalous subruns from further analysis in order to avoid any bias to our results. Fig. H.1- H.3 show the distributions studied for data quality checks. Overall, $1.7 \%$ of collected data was excluded from analysis after introducing data quality cuts.
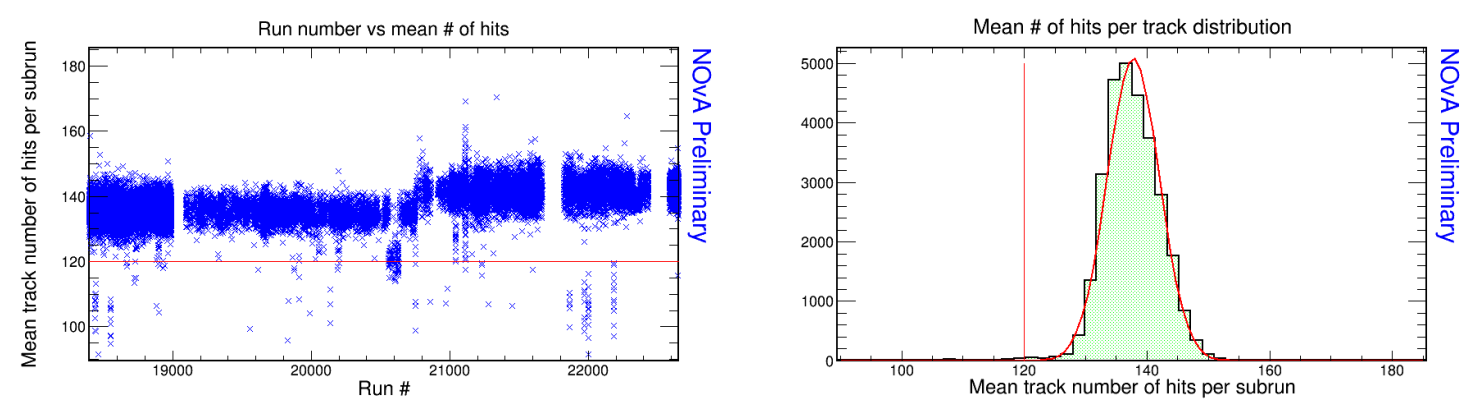

Figure H.1: Histogram of distribution of average number of hits per track by subrun of the NOvA Far Detector. Red line denotes a data quality cut on average number of hits.
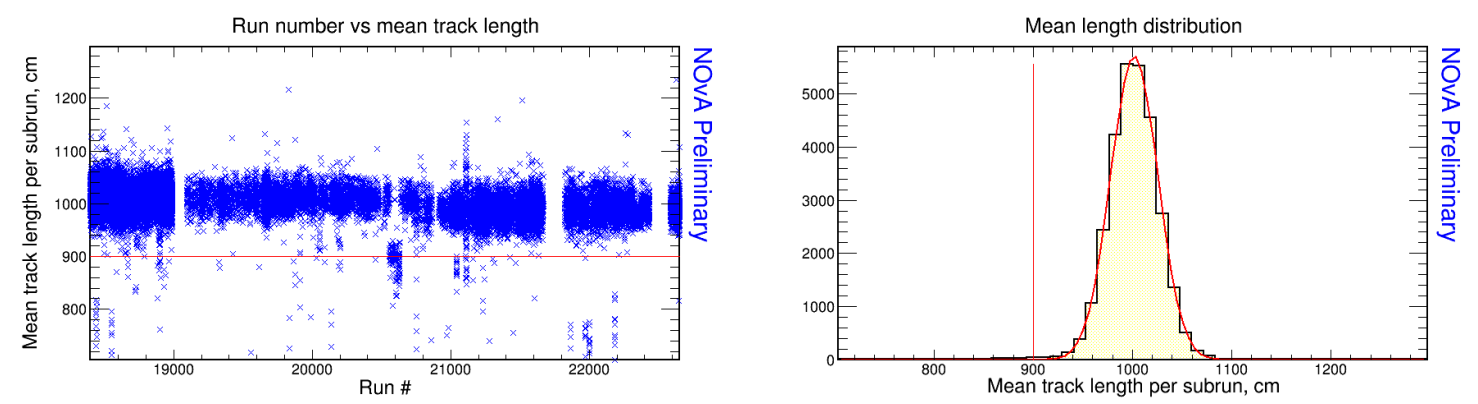

Figure H.2: Histogram of distribution of average track length by subrun of the NOvA Far Detector. Red line denotes a data quality cut on average track length. 

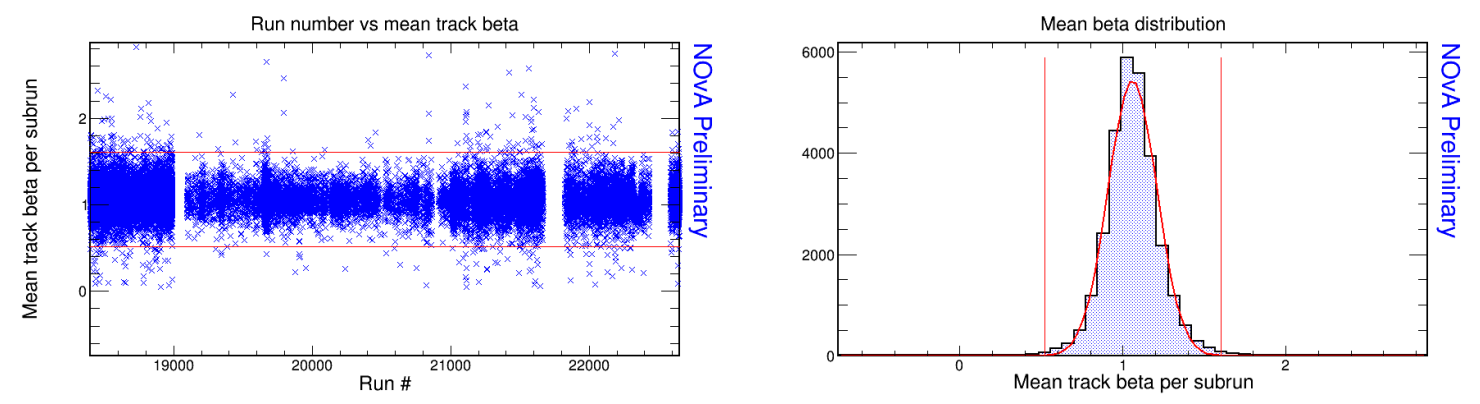

Figure H.3: Histogram of distribution of average track $\beta$ by subrun of the NOvA Far Detector. Red line denotes a data quality cut on average track $\beta$. 


\section{APPENDIX I UpMu Cut Parameters}

In this appendix cuts that were applied to data on trigger and analysis levels are presented.

Table I.1 represents cuts on track properties to select upward-going candidates on a trigger level. These cuts are less strict than corresponding analysis cuts, making trigger able to collect more data within available allocation rate.

\begin{tabular}{lll}
\hline Variable & Cut Value & Description \\
\hline TrackLen & $500.0 \mathrm{~cm}$ & 3 dimensional reconstructed track length \\
TrackHitsXY & 60 & total number of hits associated with 3D track \\
TrackHitsX & 15 & number of hits associated with XZ projection of track \\
TrackHitsY & 15 & number of hits associated with YZ projection of track \\
$\mathrm{dX}$ & 15 & Track cell length in XZ view \\
$\mathrm{dY}$ & $3 \mathrm{~m}$ & Track cell length in yz view \\
$\mathrm{dZ}$ & $3 \mathrm{~m}$ & Track plane length \\
$\mathrm{R} 2 \mathrm{X}$ & 0.99 & Coefficient of determination for fit in XZ view \\
R2Y & 0.99 & Coefficient of determination for fit in YZ view \\
Chi2 & 2.0 & Fit $\chi^{2} /$ NDF of time distribution \\
LLR & 3.0 & LLR of time distribution \\
\hline
\end{tabular}

Table I.1: Selection criteria used by the upward-going through-going muon trigger. Cuts are designed to select muon tracks with lengths and numbers of hits sufficient to compute reliable likelihood values for track direction.

Table I.1 shows track quality cuts used in the analysis module.

Table $\mathrm{I} .3$ shows efficiency of the track quality cuts shown in a previous table applied to the minimum bias cosmic pulser data.

Table I.4 shows cuts on parameters which determine track directionality. These cuts are applied in analysis module with track quality cuts I.2. Together they form the full set of cuts necessary for distinguishing reliable upward-going candidate.

Table I.5 shows the efficiency of directionality cuts applied to cosmic tracks of at least $10 \mathrm{~m}$ long with reversed directionality (i.e. the cosmic data where all tracks are considered going in opposite direction, thus majority of them going up).

Table I.6 shows a number of tracks surviving each subsequent cut in the analysed upward-going muon data sample (December 2014 to late March 2016). Final 33 events are upward-going muon candidates (some of them are misreconstructed tracks). 


\begin{tabular}{lll}
\hline Variable & Cut Value & Description \\
\hline TrackLen & $1000.0 \mathrm{~cm}$ & 3 dimensional reconstructed track length \\
TrackHitsXY & 120 & total number of hits associated with 3D track \\
TrackHitsX & 50 & number of hits associated with XZ projection of track \\
TrackHitsY & 50 & number of hits associated with YZ projection of track \\
$\mathrm{dX}$ & $3 \mathrm{~m}$ & length of track along the x direction \\
$\mathrm{dY}$ & $3 \mathrm{~m}$ & length of track along the y direction \\
$\mathrm{dZ}$ & $3 \mathrm{~m}$ & length of track along the z direction \\
R2X & 0.99 & R value from linear fit to XZ projection \\
R2Y & 0.99 & R value from linear fit to YZ projection \\
\hline
\end{tabular}

Table I.2: Track quality cuts (tracks got with window tracker algorithm). Cuts are designed to select muon tracks with lengths and numbers of hits sufficient to compute reliable likelihood values for track direction.

\begin{tabular}{llll}
\hline Cut & Initial number of events & Events after cut & Efficiency \% \\
\hline Track Length & $37,902,972$ & $16,805,471$ & $-\%$ \\
\# of hits & $16,805,471$ & $16,185,372$ & $96 \%$ \\
\# of hits/view & $16,185,372$ & $13,190,005$ & $81 \%$ \\
$\chi^{2}$ & $13,190,005$ & $12,378,344$ & $93 \%$ \\
$\chi^{2} /$ view & $12,378,344$ & $12,335,613$ & $99 \%$ \\
Track linearity/view & $12,335,613$ & $10,067,500$ & $81 \%$ \\
Length X, Y and Z & $10,067,500$ & $7,106,554$ & $70 \%$ \\
\hline \hline Combined & $16,805,471$ & $7,106,554$ & $42 \%$ \\
\hline
\end{tabular}

Table I.3: Efficiency of track quality cuts. Data is taken from the minimum bias cosmic data stream.

\begin{tabular}{lll}
\hline Cut & Cut value & Description \\
\hline$\chi^{2}$ & 1.5 & Fit $\chi^{2} / \mathrm{NDF}$ of time distribution \\
ProbUp & 0.0001 & $\chi^{2} / \mathrm{NDF}$ for the slope=1 fit \\
LLR & 5.0 & LLR of time distribution for all hits \\
LLRX & 3035985 & LLR in the X-view (3D-matched) \\
LLRY & 3035985 & LLR in the Y-view (3D-matched) \\
\hline
\end{tabular}

Table I.4: Track directionality cuts (tracks got with window tracker algorithm). Together with track quality cuts they form the full set of cuts to determine track direction. 


\begin{tabular}{llll}
\hline Cut & Initial number of events & Events after cut & Efficiency \% \\
\hline$\chi^{2}$ & 3045992 & 3035985 & $99.5 \%$ \\
ProbUp & 3035985 & 2806669 & $92 \%$ \\
LLR & 3035985 & 3003097 & $99 \%$ \\
LLRX & 3035985 & 2946254 & $97 \%$ \\
LLRY & 3035985 & 2771769 & $91 \%$ \\
\hline
\end{tabular}

Table I.5: Efficiency of the cuts

\begin{tabular}{lll}
\hline Cut & Initial number of events & Events after cut \\
\hline Length $>10 \mathrm{~m}$ & $17,250,888$ & $7,561,426$ \\
Num. of Hits $>120$ & $7,561,426$ & $7,111,648$ \\
Num. of Hits XZ and YZ view $>50$ & $7,111,648$ & $5,939,322$ \\
Chi2 $<1.5$ & $5,939,322$ & $2,299,062$ \\
Chi2X and Chi2Y $<2$ & $2,299,062$ & $2,286,412$ \\
Track linearity X and Y $>0.99$ & $2,286,412$ & $1,889,328$ \\
Length X, Y, and Z $>$ & $1,889,328$ & 1354411 \\
ProbUp $>0.0001$ & $1,354,458$ & 22,597 \\
LLR $>10$ & 22,597 & 390 \\
LLRY $>5$ & 390 & 97 \\
LLRX $>5$ & 97 & 33 \\
\hline
\end{tabular}

Table I.6: The efficiency of cuts applied to the upward-going muon data sample. 


\section{APPENDIX J \\ DDT Event Display Design and Usage}

Here the design details and usage instructions of the DDT Event Display are provided. It's sort of self-explanatory.

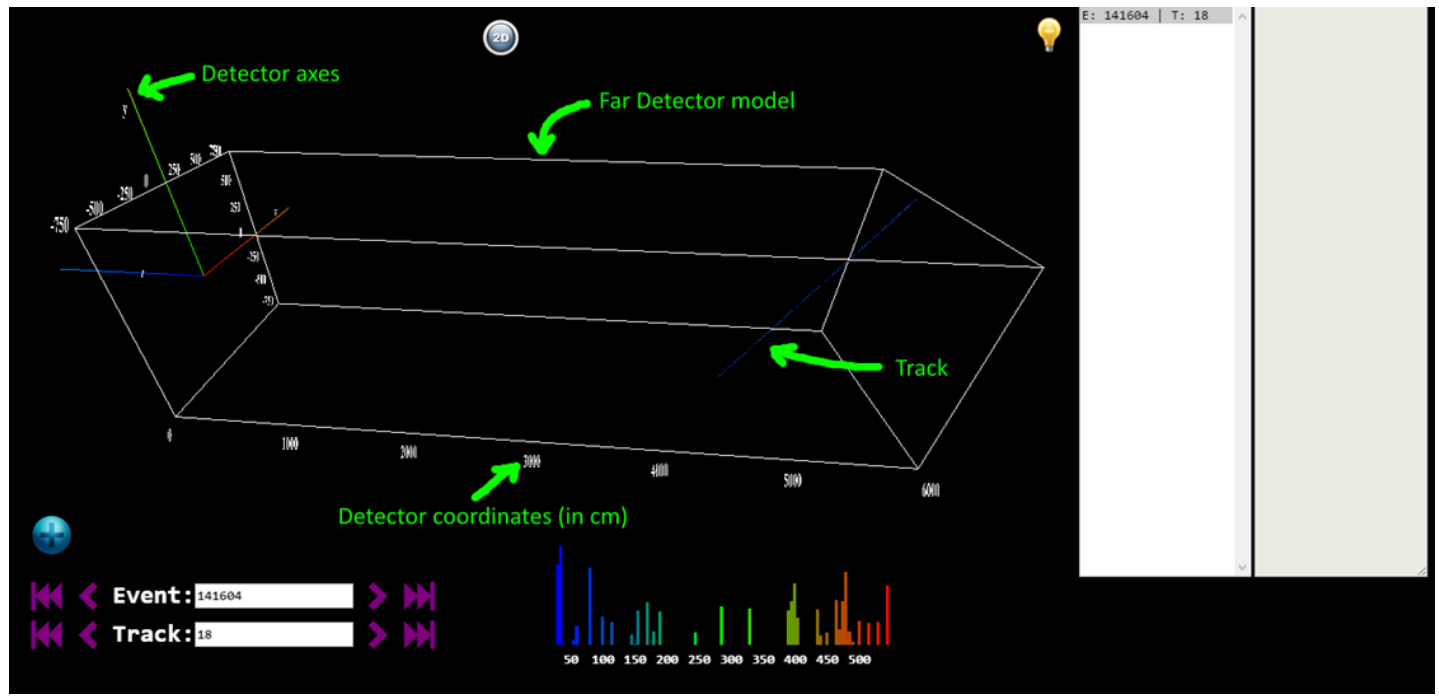

Figure J.1: Parts of the DDT Event Display: part 1

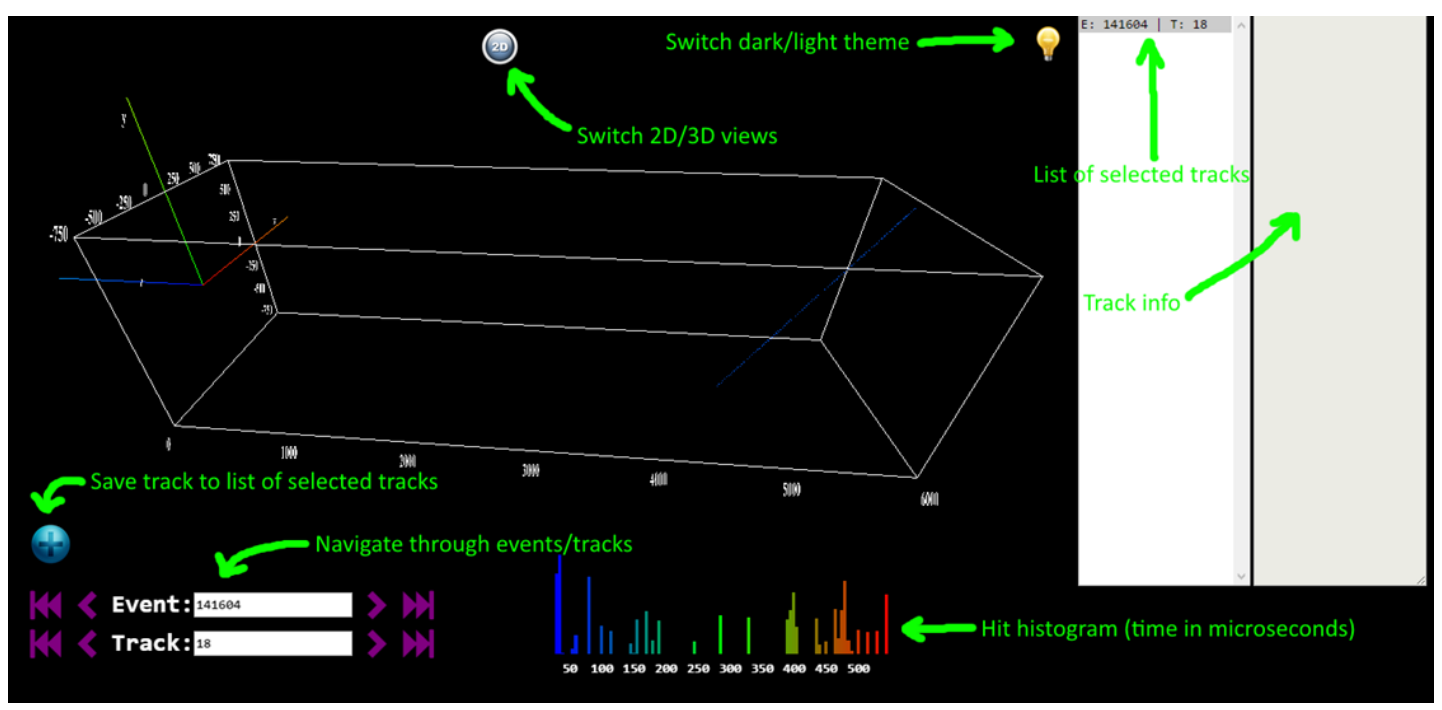

Figure J.2: Parts of the DDT Event Display: part 2 


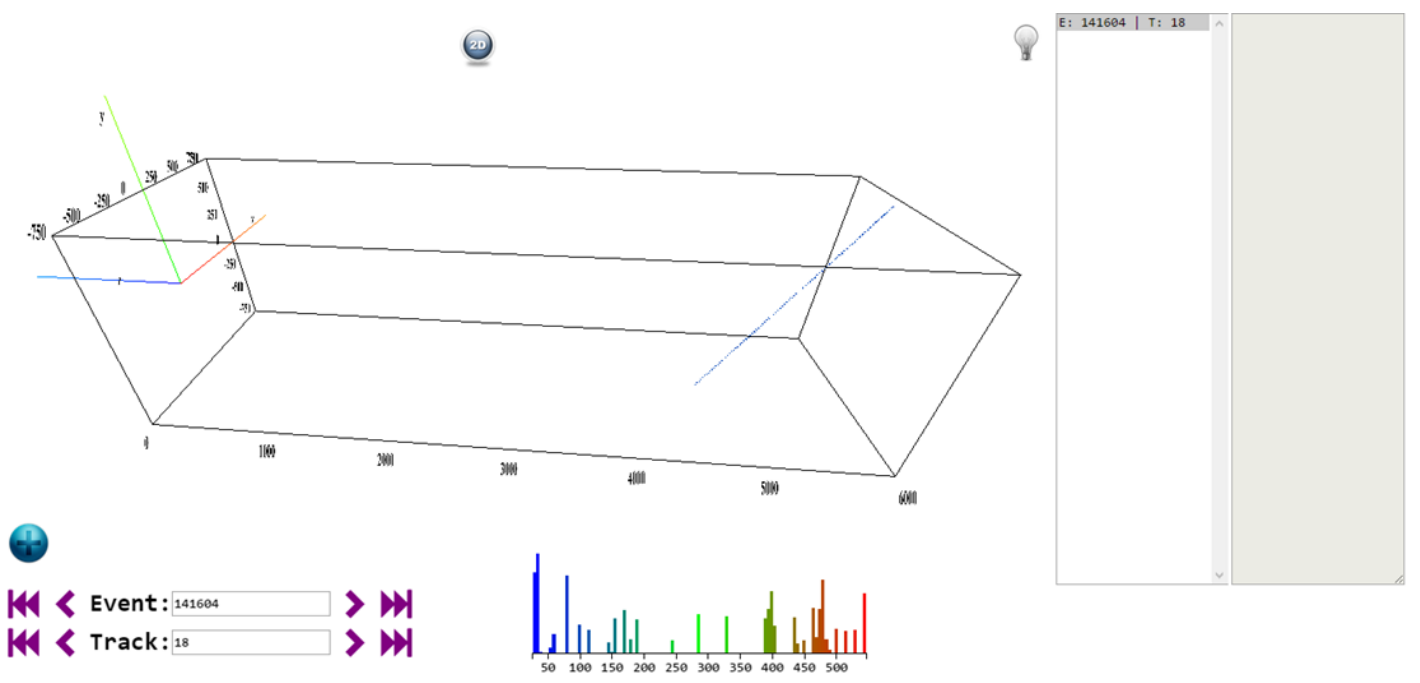

Figure J.3: The DDT Event Display with a light theme

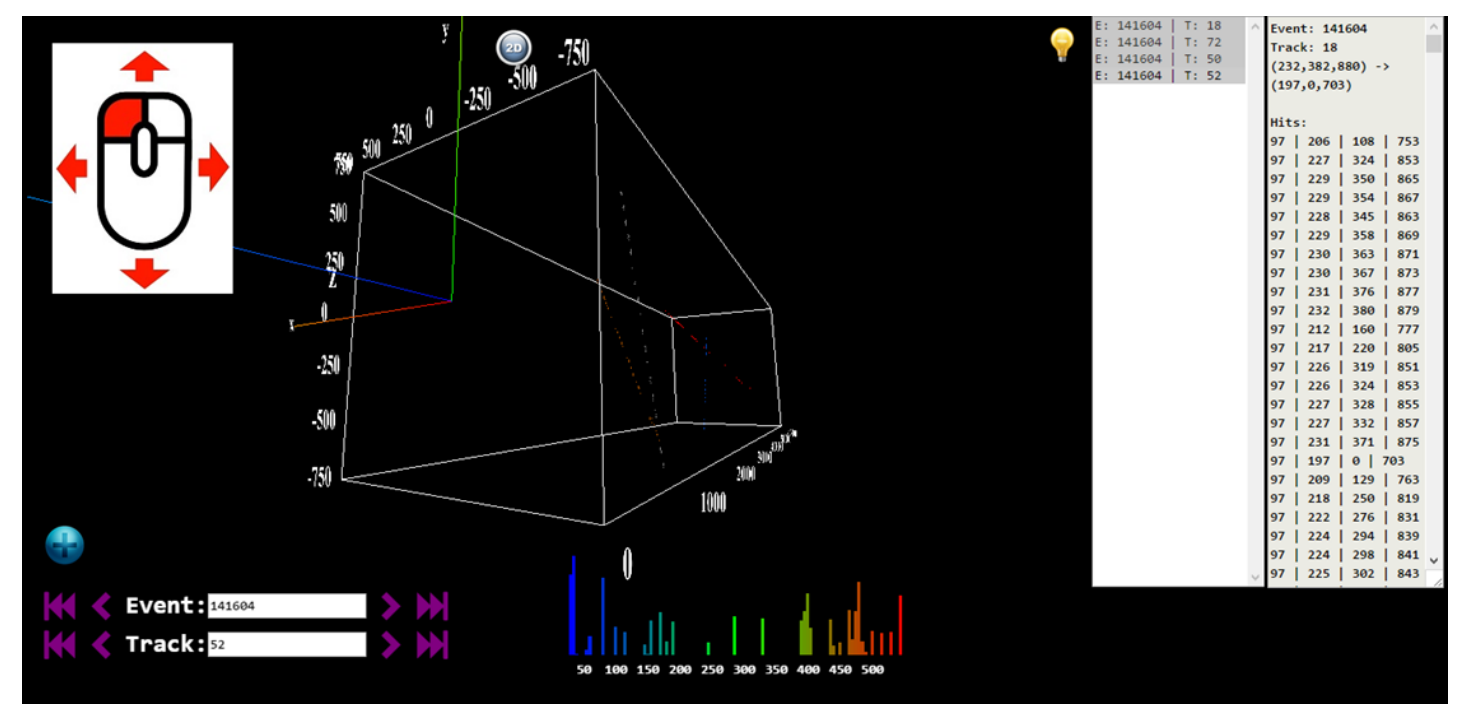

Figure J.4: How to use the DDT Event Display: rotate 


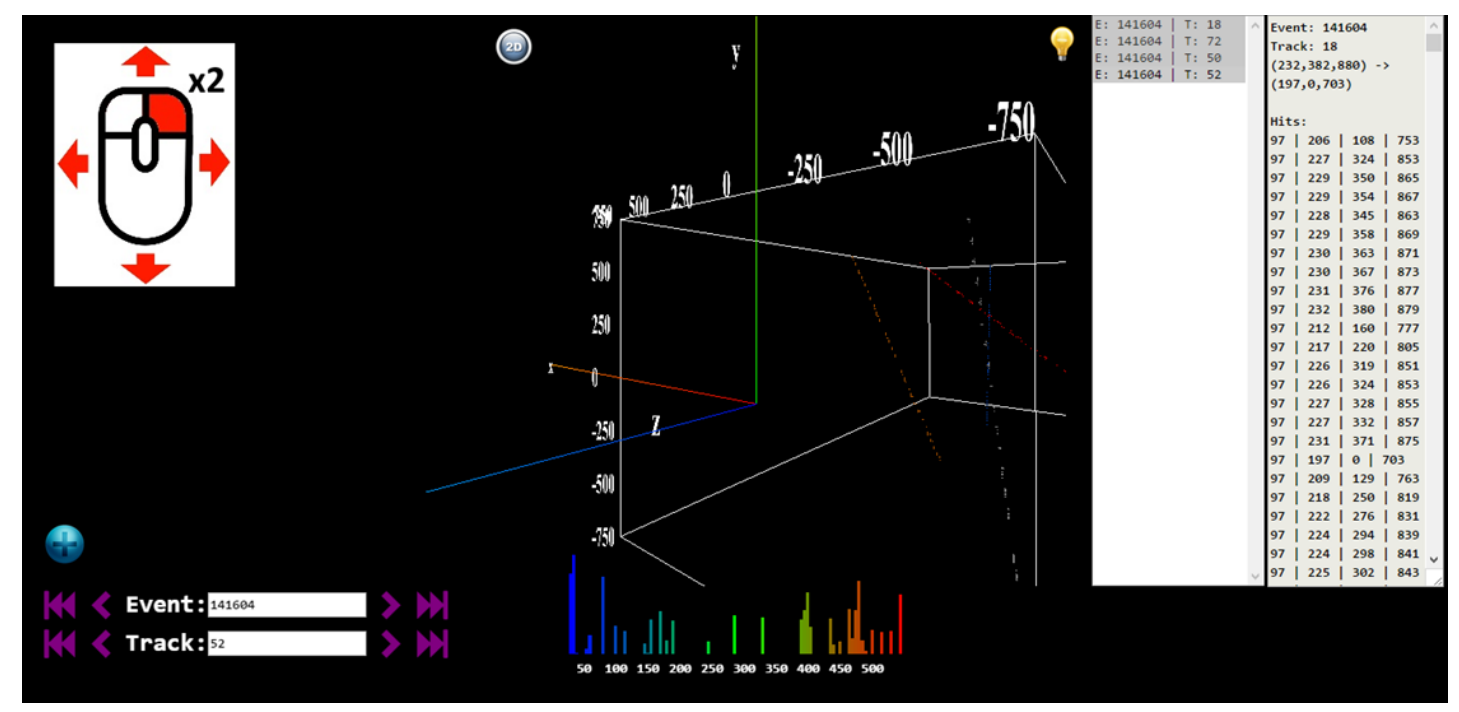

Figure J.5: How to use the DDT Event Display: move

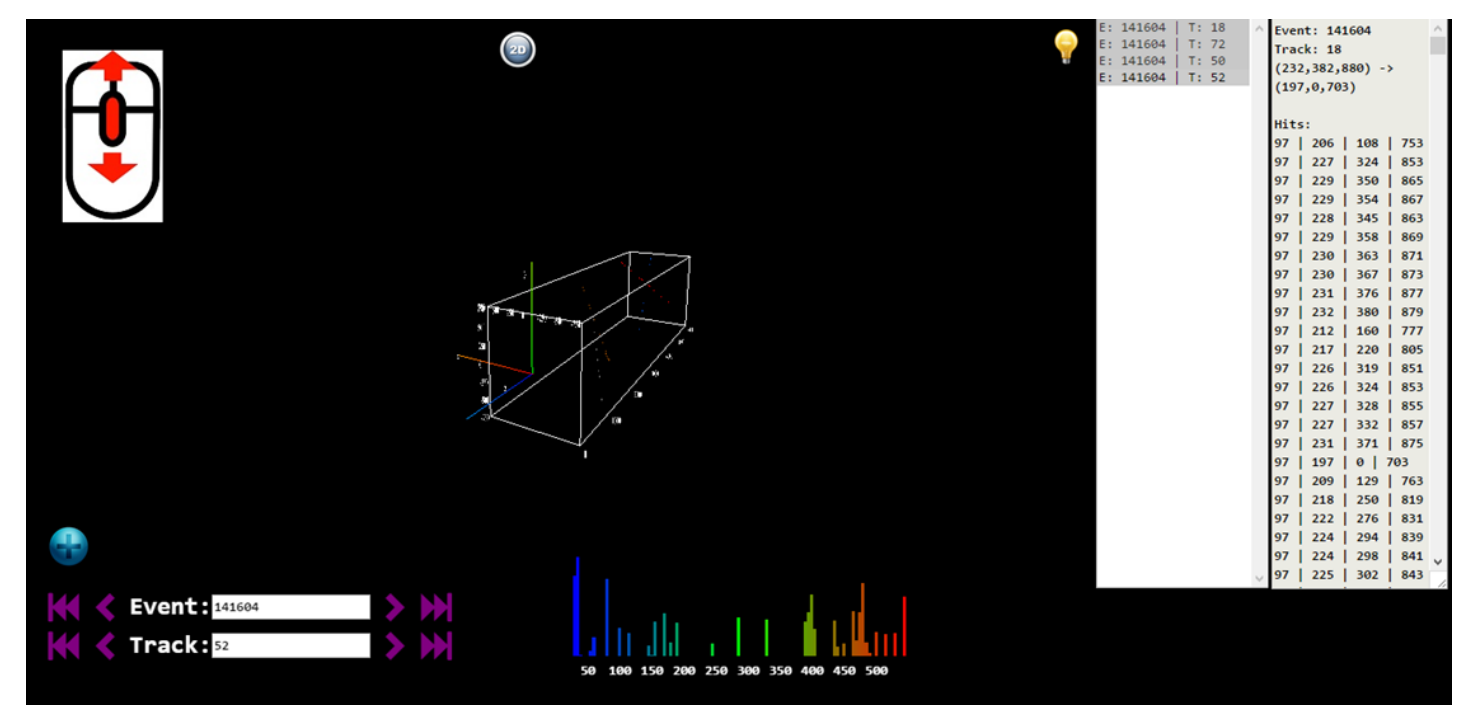

Figure J.6: How to use the DDT Event Display: zoom 


\section{APPENDIX K CSC ANATOMY}

The principal design of produced cathode strip chambers is shown on Fig. K.1.

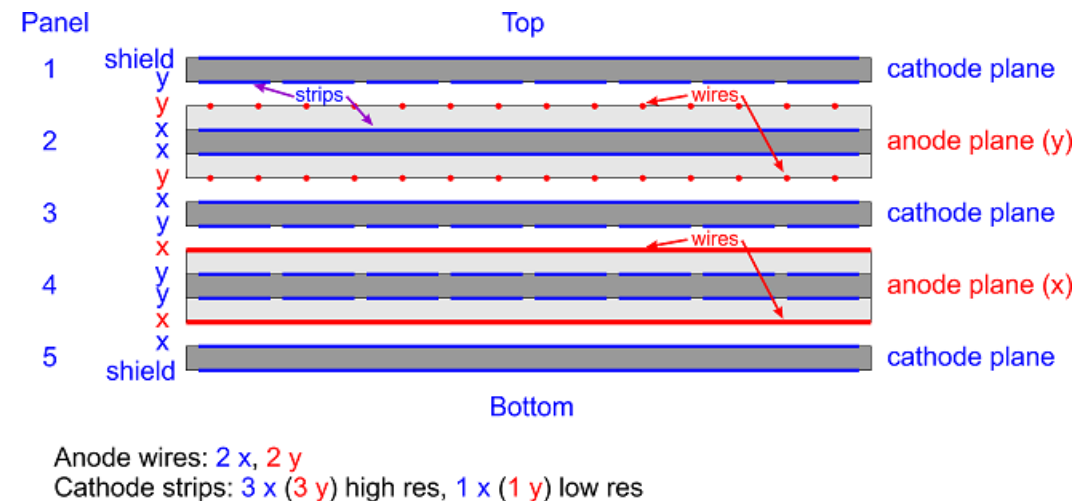

Figure K.1: The geometry of a Cathode Strip Chamber produced for Mu2e experiment

Each CSC contains 4 layers. Each layer contains 299 tungsten wires (50 $\mu \mathrm{m}$ thick) and $2 \mathrm{Be} / \mathrm{Cu}$ wires at the edges $(75 \mu \mathrm{m}$ thick). All contacts are properly soldered and divided into 15 groups in each side. Every layer has 2 sets of strips, 64 strips each, located below and above wires. Circuit scheme of a given layer is shown on Fig. K.2.

Connectors are applied to the cathode strip outputs (128 connectors for each layer in total). The connector design is shown on Fig. K.3.

The view of opened CSC layer is shown on Fig. K.4.

Thickness of assembled chambers with applied frame is measured to be $130.8 \pm$ $0.57 \mathrm{~mm}$. 


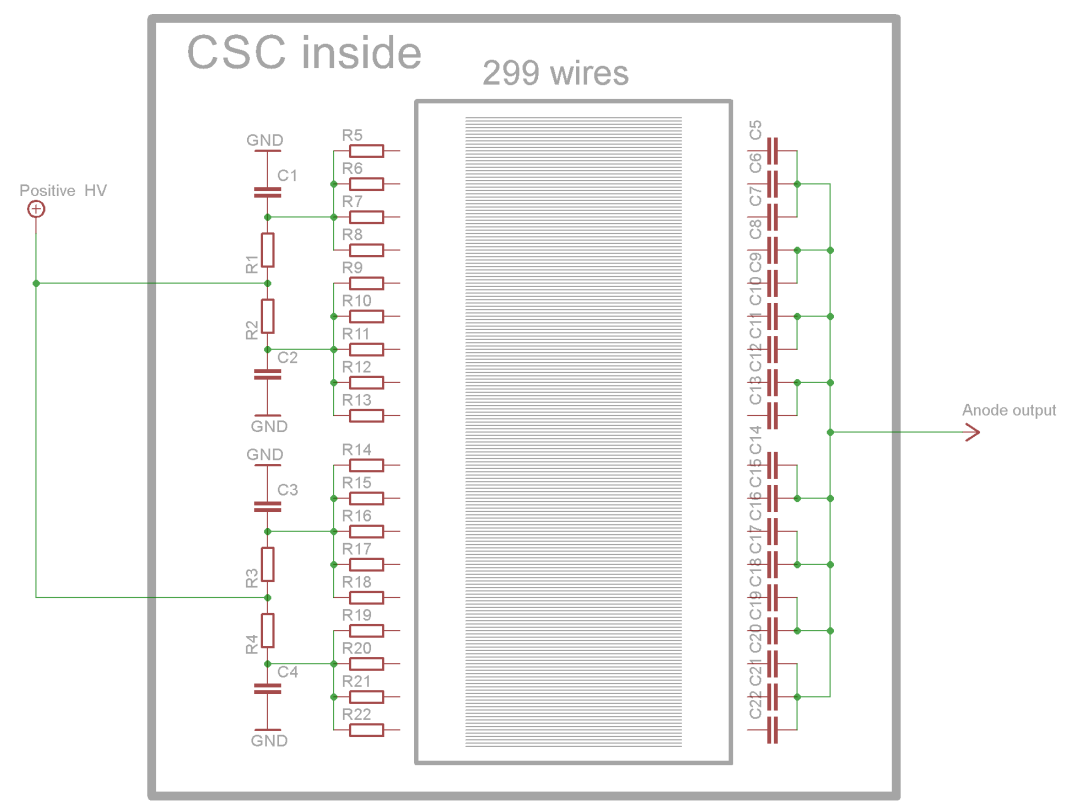

Figure K.2: The CSC circuit scheme
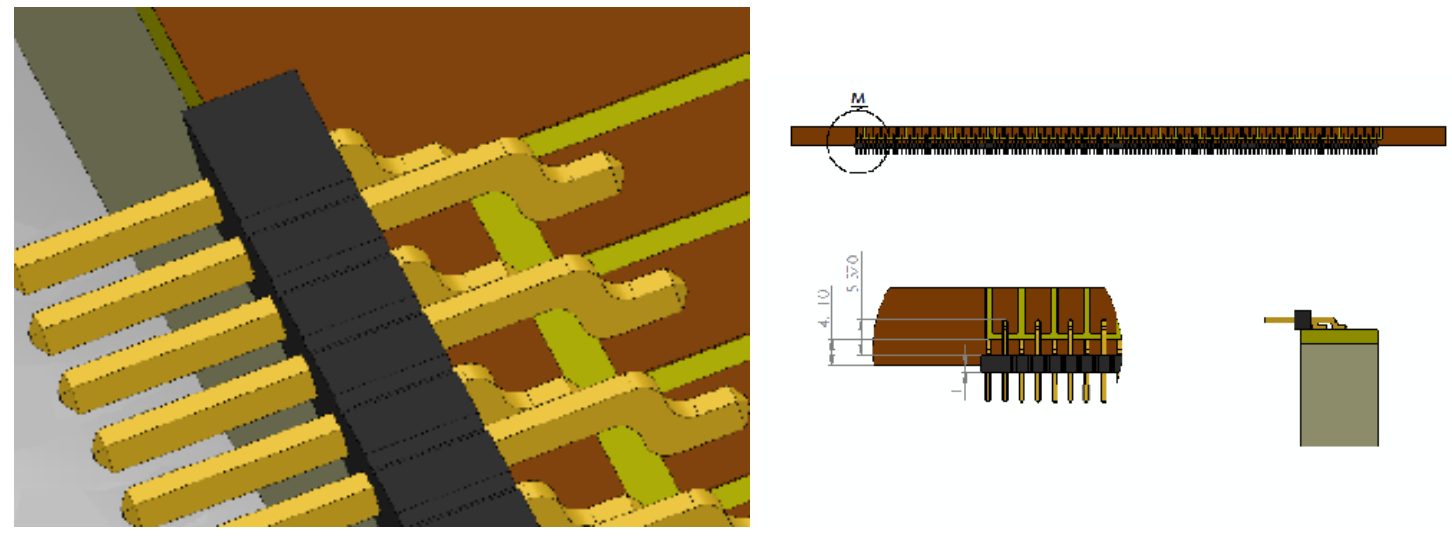

Figure K.3: The CSC cathode connectors
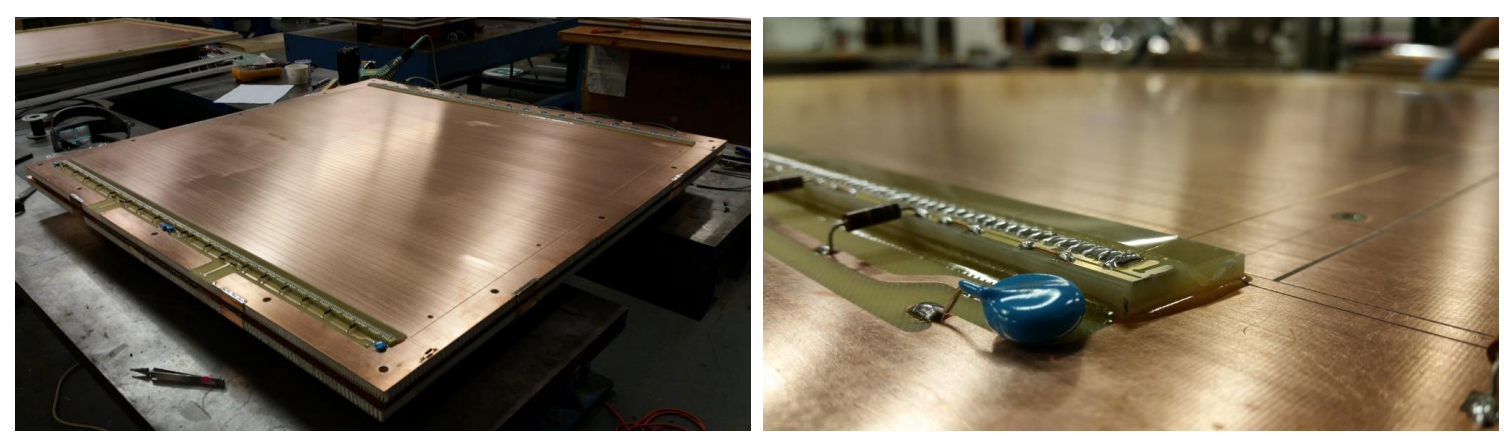

Figure K.4: One layer of an opened CSC 


\section{APPENDIX L The CSC Noise, Efficiency And Amplitudes}

Both noise and efficiency were measured separately for different planes of a cathode strip chamber under test.

Scheme used for the measurement of CSC noise characteristics is presented on Fig. L.1. Cathode outputs were connected together, so single signal from all cathodes was taken. Signal coming from cathodes passes a discriminator which digitizes the incoming signal. In addition, a gate generator was used to reduce number of false events which could be produced if a signal had trace oscillations within the discriminator gate time span.

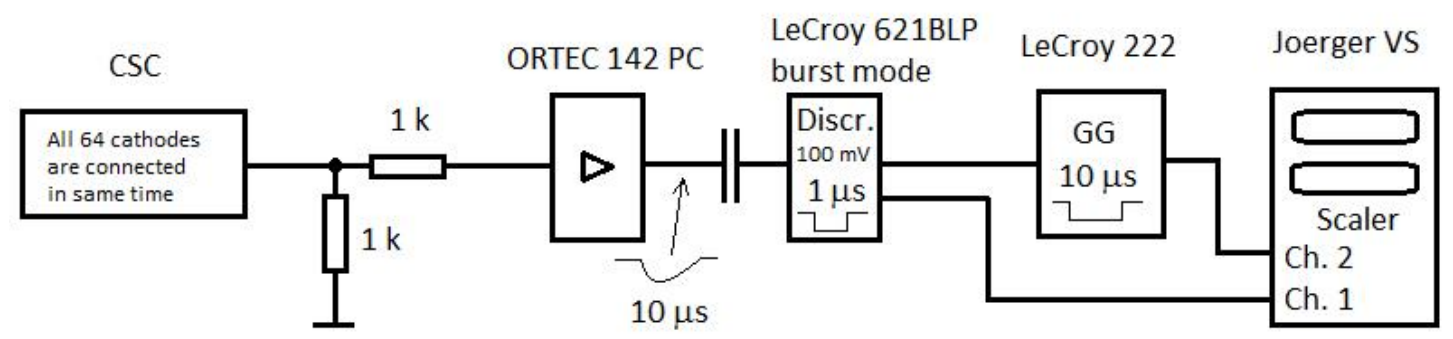

Figure L.1: The scheme used for CSC noise measurements

Provided measurements of the CSC noise show good consistency - see Fig. L.2.

For the efficiency tests one should estimate the number of events registered by a chamber compared to the total number of particles passed through a chamber. True number of events remains unknown, but we can estimate it by counting signals registered by telescope the system of reliable detectors located below and above the chamber. In this scheme the telescope plays a role of the trigger, and thus the ratio of the number of events registered by chamber and triggered by telescope to the number of events registered by the telescope only is investigated. For current tests system of two scintillators, one of which was located under the chamber and the second one on top of it, was used as a telescope.

Scheme used for the efficiency measurements is shown on Fig. L.3.

Results of the efficiency tests are shown on Fig. L.4.

For both efficiency and noise measurements, signals taken from cathodes passed through reversing amplifier with high capacitance, which sufficiently reduced noise produced by chambers. Additional capacitance at an amplifier output was used to reduce high frequency part of the noise. Proper grounding was crucial for these tests. 

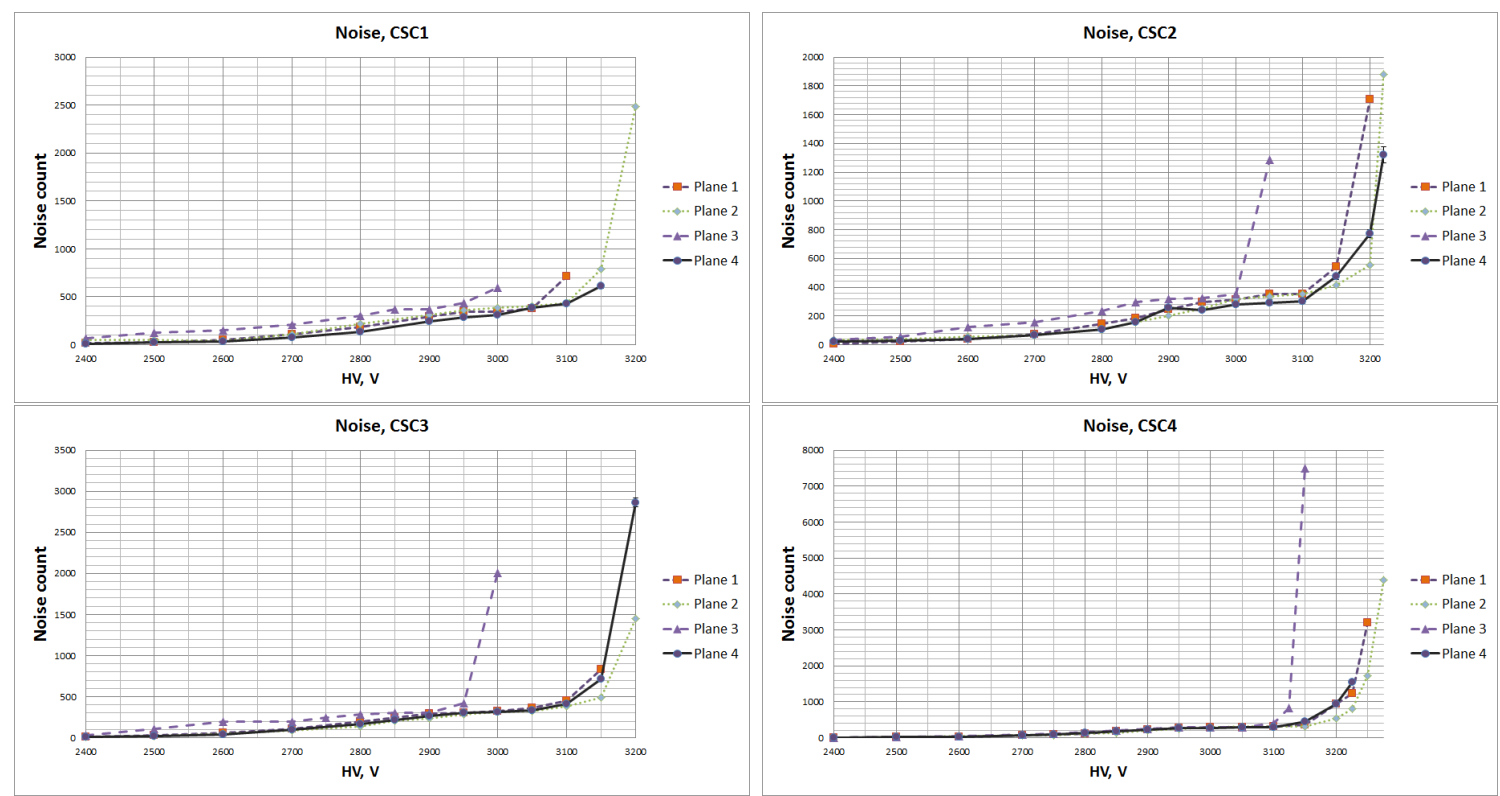

Figure L.2: The noise (in $H z$ ) of produced cathode strip chambers VS applied voltage

Especially it affected the noise characteristics measurement part, since no triggering is used for this test and bad grounding produces a lot of false signals above threshold that are noise by nature.

Measuring amplitudes of signals involved using interaction between ADC and TDC modules with a personal computer. Special program for data acquisition was created and deployed by D. Chokheli (JINR). Scheme used for signal amplitude measurements is presented on Fig. L.5.

Plots of sum of cathode signal amplitudes were made to ensure that sum of cathode signal amplitudes is close to that of anode signal - see Fig. L.6. This should be so because the charge collected by anode wires should be equal to the sum of charges collected $\mathrm{X}$ and Y strips. From these plots it is also clear that amplitude dependency on voltage is close to exponential. 


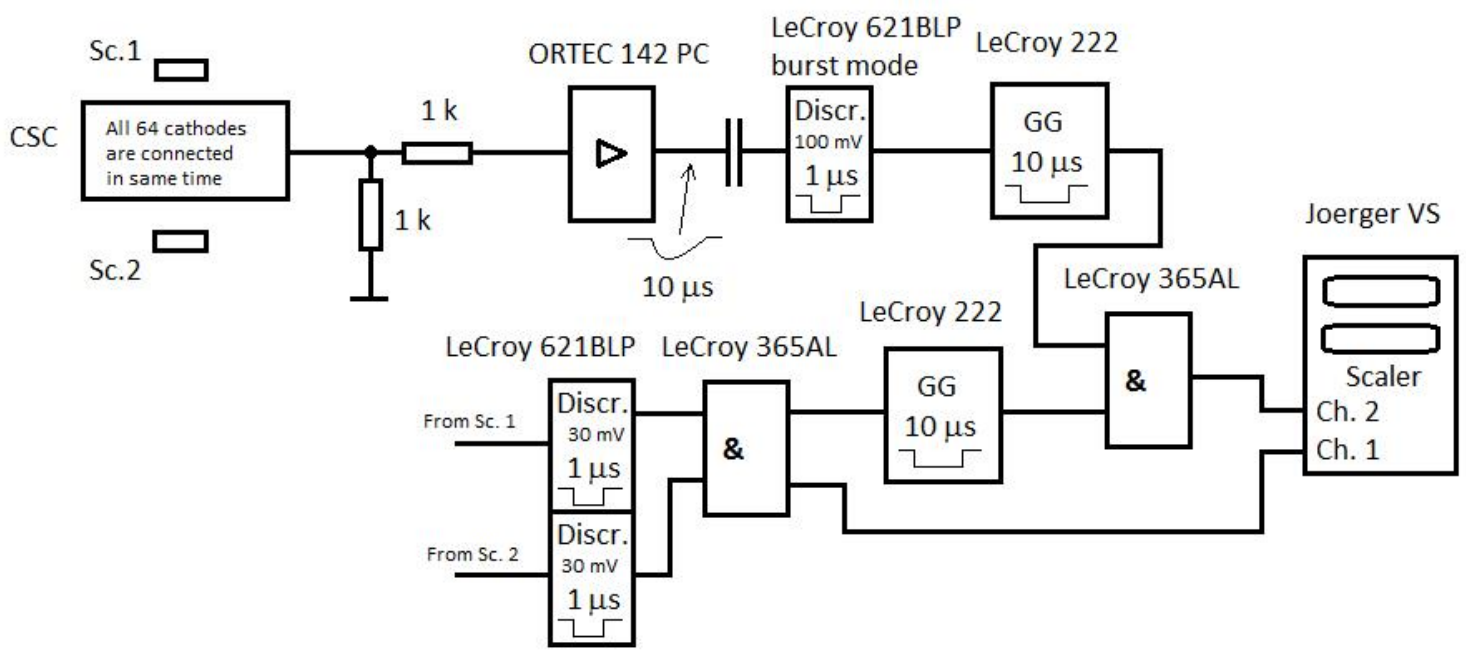

Figure L.3: The scheme used for the CSC noise measurements
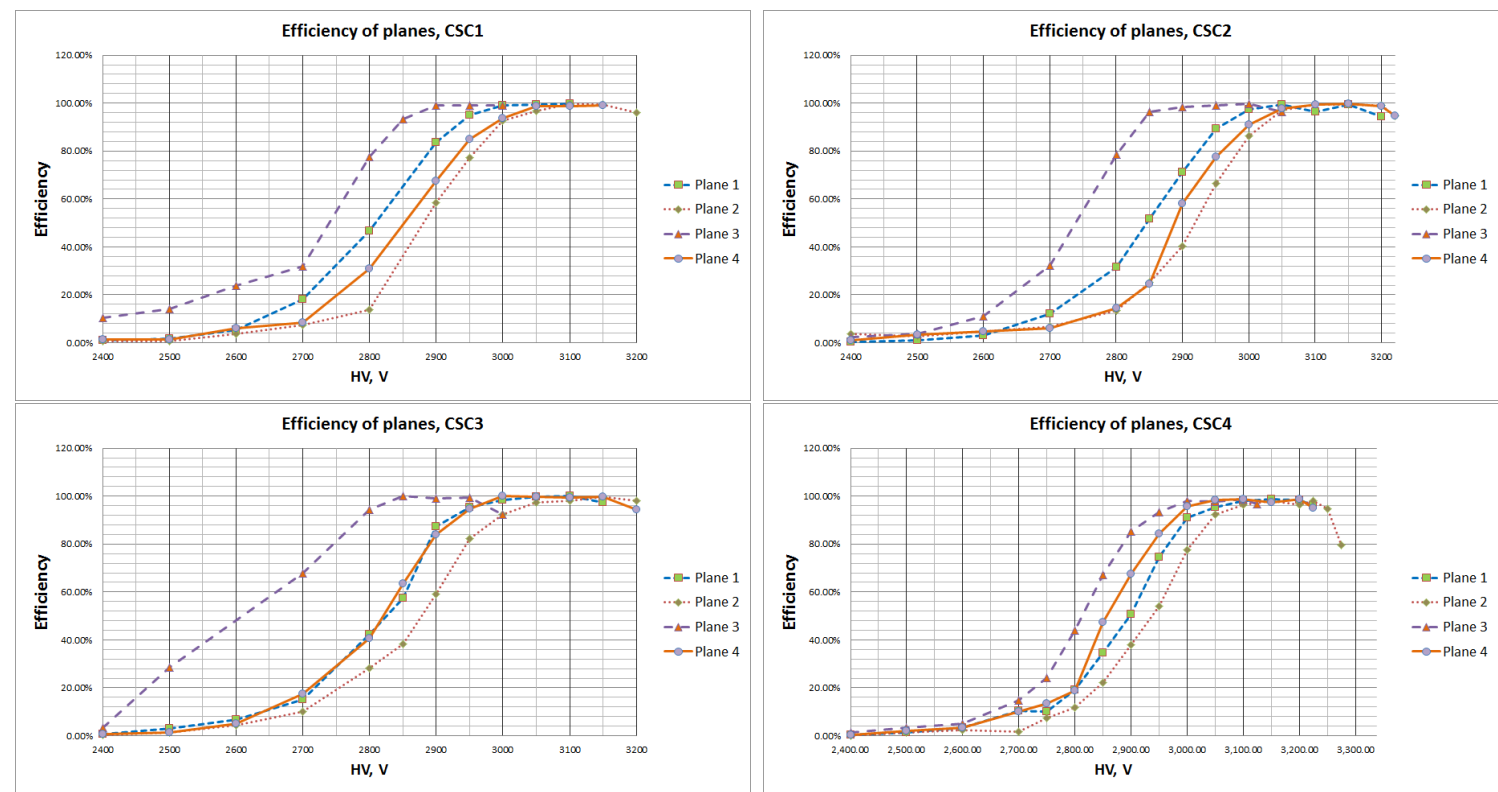

Figure L.4: The efficiencies of produced cathode strip chambers VS applied voltage 


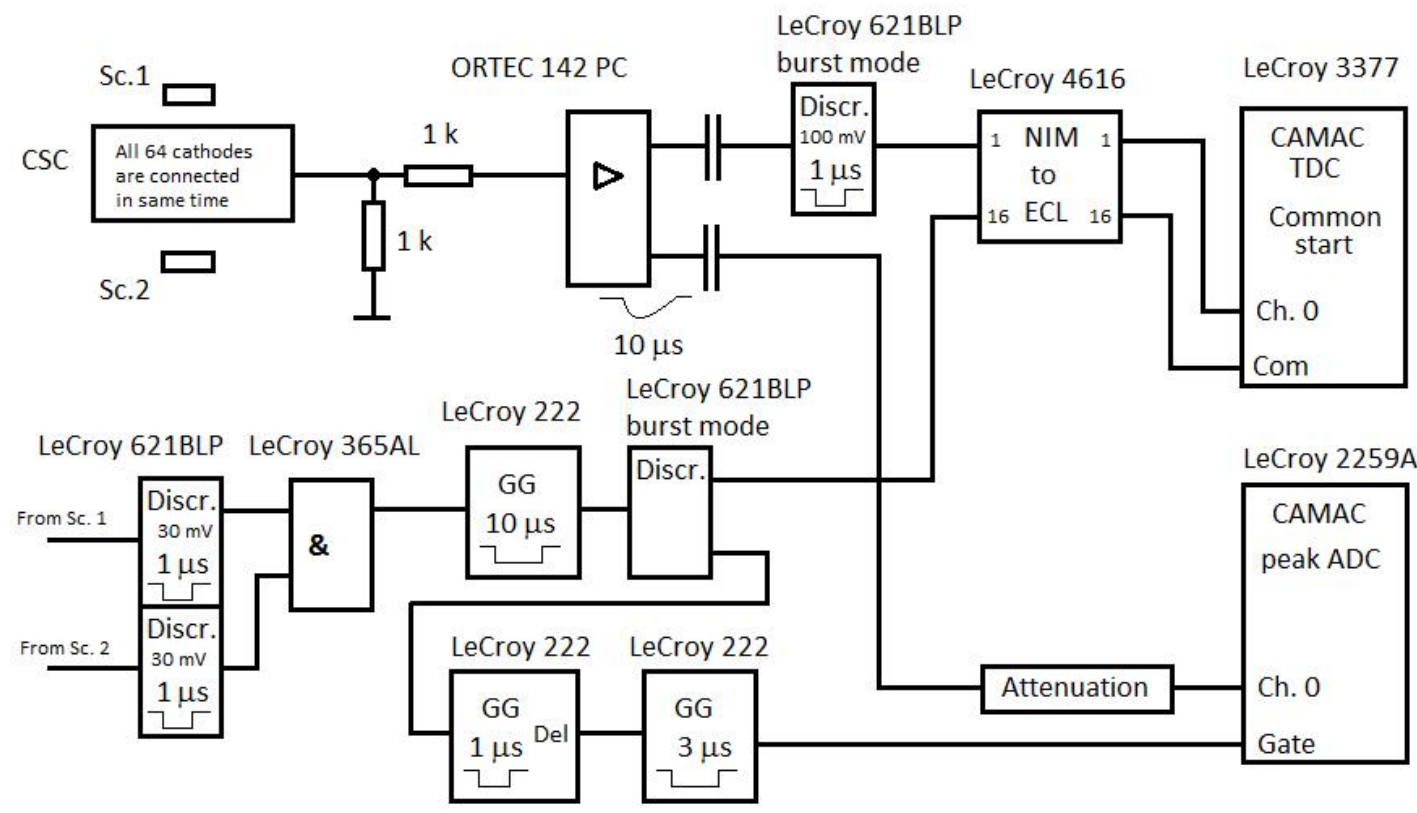

Figure L.5: Scheme used for the CSC signal amplitude measurements

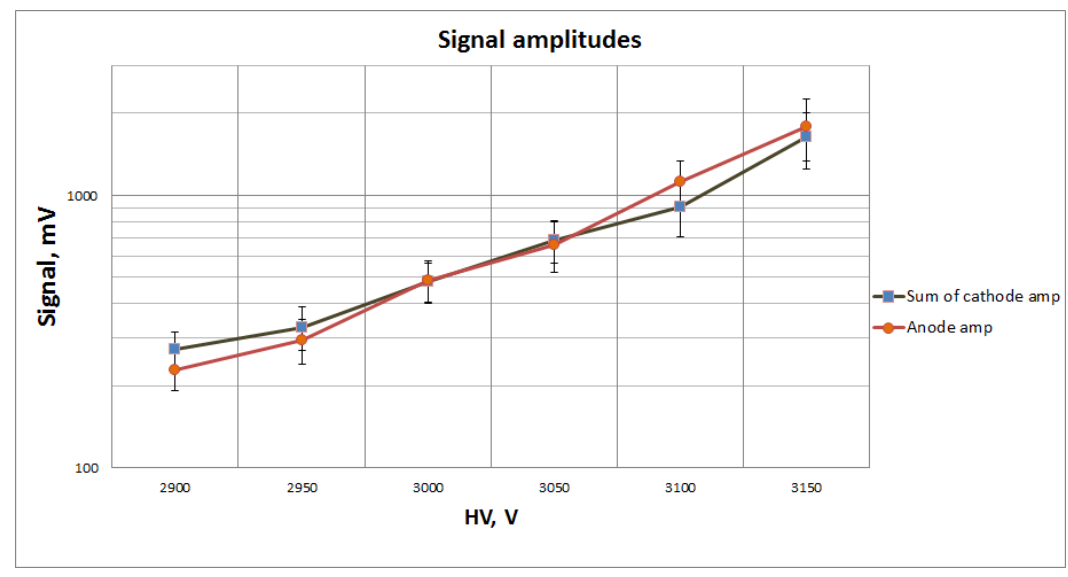

Figure L.6: Signal amplitude of selected cathode strip chamber (CSC3) VS applied voltage 


\section{REFERENCES}

[1] Fermi National Accelerator Laboratory web page

http://www. fnal.gov

[2] NOvA collaboration web page

https: //www-nova.fnal.gov

[3] ART framework web page

http: //art.fnal.gov

[4] ROOT framework web page

https://root.cern. ch

[5] Mu2e collaboration web page

https: //mu2e.fnal.gov

[6] Mu2e collaboration. Mu2e Technical Design Report.

https://arxiv.org/abs/1501.05241

[7] C. Group. Dark Matter Search Prospects at NOvA. NOvA docdb 8560 (2013).

[8] P. Adamson et al. Observation in the MINOS far detector of the shadowing of cosmic rays by the sun and moon. Astropart.Phys.34: 457-466, 2011

[9] C. Backhouse. Data driven trigger for sun/moon shadow. NOvA docdb 13225 (2015).

[10] C. Backhouse. Searching for the shadow of the moon in cosmic rays. NOvA docdb 13221 (2015).

[11] K.A. Olive et al. (Particle Data Group)

Chin. Phys. C, 38, 090001 (2014) and 2015 update.

[12] Moon Trigger repository

https://cdcvs.fnal.gov/redmine/projects/novaddt/repository/ entry/trunk/MoonShadowTrigger

[13] Naval Observatory Vector Astrometry Software (NOVAS)

http: //aa. usno. navy. mil/software/novas/novas_py/novaspy_ intro.php.

[14] NOVAS guide for C library

http://aa. usno.navy. mil/software/novas/novas_c/NOVAS_C3.1_ Guide.pdf 
[15] NASA ephemeride files

http://ssd.jpl.nasa.gov/?planet_eph_export

[16] CelestialLocator Service repository

https://cdcvs.fnal.gov/redmine/projects/novaart/repository/ show/trunk/CelestialLocator

[17] M. G. Aartsen et al. [IceCube Collaboration]. Phys. Rev. Lett. 110, no. 485 13, 131302 (2013).

[18] T. Tanaka et al. [Super-Kamiokande Collaboration]. Astrophys. J. 742, 48778 (2011).

[19] K. Choi et al. [Super-Kamiokande Collaboration]. Phys. Rev. Lett. 114, 489 no. 14, 141301 (2015).

[20] M. Blennow, J. Edsjo, T. Ohlsson. JCAP 0801, 021 (2008).

[21] J. Edsjo. WimpSim Neutrino Monte Carlo .

http://www. fysik. su.se/ed-sjo/wimpsim/

[22] R. Mina et al. Implementation of an upward-going muon trigger for indirect Dark Matter searches at the NOvA far detector. CHEP2015, Okinawa, Japan, 13-17 April, 2015. Pre-print.

[23] Upward-going muons trigger repository

https://cdcvs.fnal.gov/redmine/projects/novaddt/repository/ show/trunk/ExoticsTriggers

[24] Upward-going muons analysis modules repository https://cdcus.fnal.gov/redmine/projects/novaart/repository/ show/trunk/Eval

[25] T.K. Gaisser, M. Honda. Flux of Atmospheric Neutrinos. Ann. Rev. Nucl. Part. Sci. 52: 153-199, 2002

[26] M.C. Sanchez. Invited talk at the XXXI Conference on Physics in Collision, Vancouver, Canada, 2011.

https://arxiv.org/abs/1112.0618

[27] D.E. Groom, N.V. Mokhov, S. Striganov (Particle Data Group). Muon Stopping Power and Range Tables .

http://pdg. lbl.gov/2015/AtomicNuclearProperties/adndt.pdf

[28] The Super-Kamiokande Collaboration. Measurement of the flux and zenithangle distribution of upward through-going muons by Super-Kamiokande. Phys. Rev. Lett. 82: 2644-2648, 1999 
[29] Artdaq Event Display repository

https://cdcus.fnal.gov/redmine/projects/novaart/repository/

show/trunk/EventDisplay

[30] DDT Event Display data module repository (offline)

https://cdcus.fnal.gov/redmine/projects/novaart/repository/ show/trunk/DDTEventDisplay

[31] DDT Event Display on Fermilab personal page

http://home.fnal.gov/〜serdar/ddt_evd.html

[32] O. Prokofiev, P. Yamin. CSC Prototypes Construction for Mu2e Experiment. Mu2e Doc DB 3683, 09/09/2013. 